\title{
THE HIPPOCAMPUS AND ITS RELATIONS TO THE CORPUS CALLOSUM
}

\author{
FREDERICK TILNEY, M.D.
}

1. Although many picturesque and useful anatomical names have been the fruit of Renaissance ingenuity, such adventures in nomenclature were not always happy. Often enough the resurgent imagination was allowed too loose a rein. The term hippocampus, first applied in 1587 by Julius Caesar Arantius to a widely discussed region of the brain, is a notable case in point. Since the time of Arantius' suggestion no one has been able to discover any resemblance, striking or remote, between the part of the brain so labelled and the little sea-horse fish known as hippocampus.

Frederick Lewis goes so far as to say that "the flight of fancy which led Arantius to introduce the term, hippocampus is recorded in what is perhaps the worst anatomical description extant."

Not only is this original description of the hippocampus thoroughly unsatisfactory, but the effect of such a name applied to cerebral structures has resulted in unnecessary confusion. Yet, in fairness to Arantius, it must be said that he was not too strongly biased in his proposal. A quotation from his brief chapter on this subject shows a considerable degree of openmindedness. "This recalls the image of a Hippocampus, that is, of a little sea-horse. Rather, perhaps, it suggests the form of a white silkworm (Bombycinus vermis)." On the basis of structural similarity there is little to recommend either hippocampus or bombyx. It was some whim of popular favor rather then any insistency on the part of Arantius which preserved one term and discarded the other, for, whatever its faults, hippocampus has now received the approval of almost universal usage. ${ }^{1}$

I I am indebted to Doctor Samuel W. Lambert for the following translation of Arantius' description of the hippocampus, which under the title of "De cerebri particulis Hippocampum referentibus" appears in Arantius. De humano foetu... Ejusdem anatomicorum observationum liber, etc. Venetiis, 1587, p. 44-45.

"Horum ventriculorum basi, quae intro ad medium respicit, candida insurgens supereminet, \& quasi adnascitur substantia, quae ab inferiori superficie, uelut additamentum extollitur, psalloidique corpori, seu testudini est continua, ac per longitudinem, in anteriora, uersus frontem protenditur inaequalique, ac flexuosa figura praedita est, quae Hippocampi, hoc est marini equuli effigiem refert, vel potius, bombycini vermis candidi spinalis medullae initium hine inde amplexantis, formam indicant, de cuius vsu alibi dicemus; huius particula caput referens tertio vocato ventriculo proxima est, reflexum uero corpus in caudam abiens, ad anteriora protenditur; quo circa ad superiorum differentiam, Hippocampi, vel Bombycini vermis 
Objections to it have been raised from time to time during the past three hundred years. Some proposals for substitutes have also been offered but, unfortunately, without much in the way of improvement. Winslow's effort at reform in 1732 was largely futile. He saw in this region of the brain a strong likeness to a ram's horn and proposed the term Cornu arietis. Ten years later Garengeot (1742), for no particular reason, gave this horn idea certain lasting qualities by injecting a mythological flavor into it. With quite gratuitous flattery for an Egyptian God of first rank he offered to call this portion of the cerebrum the Cornu Ammonis. The god Amun Kneph, with his noted temple in the Libyan desert, did especially well at the hands of medicine. In addition to this famous horn in the brain, he is memorialized by "The gum and sal ammoniac gathered from trees and camel dung" (Hyrtl). But the Cornu Ammonis was destined to go a long way despite its ridiculous origin. It was adopted by many earlier anatomists and is still used with confidence by many modern authors.

ventriculos appellare libuit. Illud praeterea obseruatione dignum relinquitur, quod plexu e sinuum basi sublato, elegans quaedam, atque admirabilis, exiguorum quamuis vasorum, propagatio conspicitur, quae in superioribus non est adeo artificiosa. Quod ad cavitates, \& religua, sic habet.

The following translation is by Dr. Samuel W. Lambert.

"At the lowest part of these ventricles a white structure rises up and projects above as if it were an additional grow th which faces internally and backwards toward the middle line. This is continuous from its lower surfaces with the psalloid body or lyra and throughout its length anteriorly is stretched out unevenly and is provided with a curved form which brings back a resemblance to the little marine horse: the hippocampus, or rather to the white worm, the Bombyx, and points out the form of the beginning spinal medulla coiling around its beginning. We will speak concerning the use of this latter in another place.

The small portion of this hippocampus carrying the head is next to the ventricle called the third, in fact the body running to the tail is extended (forwards) at that place in which is the division from the upper parts and it was pleasing to name those the ventricles of the Hippocampus, or of the silk worm. Besides this remains worthy of notice whenever the plexus is removed from the base of the sinuses that a certain fine and noteworthy extension of markedly small vessels is observed which are not so delicate in the upper parts. Because it is so arranged for the cavities and the rest of these structures.

Commenting on Arantius' chapter, Doctor Lambert says, "It is always difficult translating anatomical Latin of the 16th century not to twist the author to fit in with our modern ideas and to give the authors credit for more imagination than any of them had. You will remember that the physiology of the 16th eentury laid stress on the cavities of the organs, heart, brain, lungs, kidney, ete., and not on the solid walls. So far as the brain was concerned, the cavities were the essential portion and by the pulsation of the brain distributed through the nerves a humor known as animal spirit, animal referring to anima, the soul and not to animal, the brute. This was distributed through the nerves which had channels in them through which this elusive "hormone" could travel, although these chamnels were not visible to the human eye." 
In the course of time, the original hippocampal idea received some cmbellishments. The pes hippocampi (major and minor) as well as hippocampal digitations were described and to these was added Morand's bird's claw (calcar avis-1744). All in all the hippocampus of the brain had now become a weird creation which, starting as a fish, gradually acquired feet, toes and claws. The biological dilemma was obvious. One way out of the embarrassment was suggested by Mayer in 1799, who did not hesitate to call upon an amphibious quadriped mammal and designate this part of the brain the pes hippopotami.

Having thus run almost the entire gamut of the vertebrate phylum in its onomatology, it only remained for the hippocampus to receive the blessing of the Nomina Anatomica adopted in Basle (1895). According to this commitment Cornu Ammonis is proseribed, pes hippocampi is excluded, but strangely enough, calcar avis is found acceptable, while the hippocampus is sanctioned and retains its original prominence in full.

\section{CIRCUMVENTION OF DIFFICULTIES IN NOMENCLATURE}

The inconveniences of Arantius' time honored conception are clearly long past effective revision. Furthermore, it seems possible to overcome these difficulties by defining precisely what is meant by this part of the brain and its several constituents. In the following descriptions hippocampus and hippocampal formation refer to one of the four pallial differentiations of the cerebral hemisphere, namely, the archipallium. This division of the pallium is, as its name implies, of greatest antiquity. It occupies a position along the lower margin of the mesial surface of the cerebral hemisphere and extends in a sweeping arch from the lower part of the frontal region, above the optic thalamus and thence downward into the basal portion of the temporal region. It is especially distinguished by the characteristics of its embryonic development. In this respect it differs strikingly from the three other divisions of the cerebral pallium. The several successive stages of embryonic development, therefore, afford valuable guides in defining the limits and structures of the hippocampus. My chief aim in these descriptions is to disclose the unitary structural nature of the archipallium among the mammals and thus to establish a new basis for discussing the functions of the hippocampus. For special definitions of structures entering into this part of the brain, I am indebted to Dr. Marion Hines, whose excellent studies on the hippocampal region I have found invaluable guides.

A particular feature, which has given rise to considerable confusion in many descriptions, is the introduction of the corpus callosum into this region of the brain. On the other hand, the presence of this commissural body lends an especially stimulating interest to the study of the hippocampus. 


\section{METHOD AND MATERTAL}

The disadvantages of studies based exclusively either upon comparative anatomy or upon comparative embryology have often been emphasized. It seemed desirable, therefore, in this investigation to carry on both explorations hand in hand in order to obtain as broad a survey of the field as possible. Inasmuch as one of the interesting problems centers about the relations of the corpus callosum to the hippocampus, the study was limited to the mammals although the great significance of the reptilian brain with reference to the archipallium cannot be overlooked.

In order to give uniformity to the investigation, wax plate reconstructions were made of each brain studied. This method permitted the use of different colored wax plates to represent the several different areas and constituents of the hippocampus.

Embryological studies were confined to closely placed series of six mammalian species including the opossum, rat, guinea pig, common pig, cat and man. Sections were cut in the coronal and horizontal planes and stained with hematoxylin-orange $G$., hematoxylin-eosin and cresyl-violet.

Serial sections of a number of adult mammalian brains, stained by the Pal-Weigert method were also studied and, in several instances, reconstructed. The plane of section in all such cases was horizontal. These adult brains included the kangaroo, mole, rat, cat, dog, giraffe, tapir, horse, lemur, marmoset, South American monkey, macacus, baboon, gibbon, orang-outang, chimpanzee, gorilla and man. The microscopic studies were supplemented by gross dissections of the opossum, rat, cat, dog, elephant, deer and man.

The absence of monotremes, edentates and chiropteres from this list of species leaves an unfortunate but unavoidable gap due to lack of such specimens.

\section{THE HIPPOCAMPUS IN ITS SIMPLEST MAMMALIAN FOIRM}

The hippocampus in most mammals is complicated by its intimate relations with the corpus callosum. The easiest way of explaining these relations is to begin with a mammalian brain which has no corpus callosum. The Virginia opossum possesses several advantages for this purpose. Not only is the hippocampus comparatively simple, but the animal as a whole is so primitive that it has been called a "living fossil." This marsupial is the most ancient of extant, viviparous mammals. Its skeletal structure has remained almost entirely unaltered since the Age of Reptiles. Yet in spite of this extreme primitiveness, it follows, stage for stage, the fundamental principles which underlie the growth of the brain in all mammals. This feature of its development is perticularly striking in the origin and growth of its cerebral cortex.

The steps in this process are worth following closely. Immediately after 
the mantle layer makes its appearance in the endbrain, the cerebral hemispheres pass through the successive phases of general, divisional and local cortical differentiation. Each cortical division follows a mode of specialization which is strictly its own and invariable, in all essential details, from the lowest to the highest mammals. Even in the early stages of growth, the divisional boundaries between the archicortex (hippocampus), paleocortex, bulbar cortex and neocortex may be easily discerned.

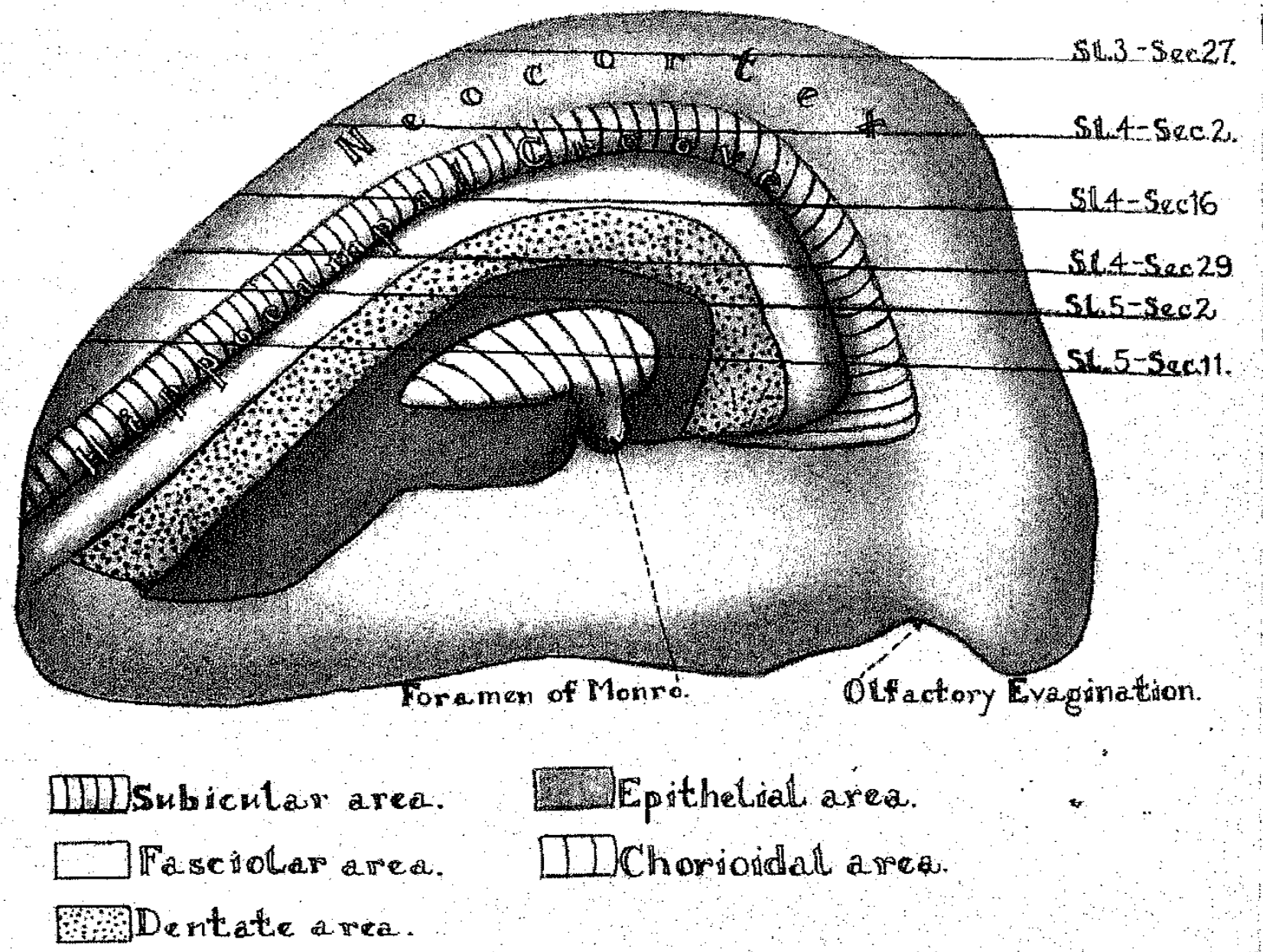

Fia. 1. Reconstruction Showing Mesial Surface of Left Hemisphere in 16 ma. Pouch Youna Opossum

Specimen No. 2005. Magnification $\times 70$

Five arches of the hippocampal region in 16 millimeter pouch young opossum

In the $16 \mathrm{~mm}$. pouch young opossum, the region which contains the primordial elements of the hippocampus is sharply defined. It forms an arch along the lower border of the mesial surface of the cerebral hemisphere. From the standpoint of cellular organization this arch actually consists of five superimposed arches (fig. 1).

The first or outer arch extends upward from the basal portion of the frontal region, backward above the optic thalamus and then downward into the base of the caudal region. The upper border, throughout the entire extent 
of this arch, is continuous with the neocortex. No surface boundary line marks the transition from the neocortex to this outer portion of the hippocampal arch. Cellular difference in these two areas do make a clear distinction between them.

The second arch occupies a position subjacent to the first. In regular order, the third arch is situated subjacent to the second, the fourth to the third and the fifth to the fourth. The positions and relations of these five arches are shown in a reconstruction of the mesial surface of the hemisphere in the $16 \mathrm{~mm}$. pouch young opossum (fig. 1).

No markings on this surface of the brain as yet indicate the limits of the separate arches. On the other hand, the differences in their cellular specializations are pronounced enough to determine five distinct areas in the brain wall. In view of ultimate differentiation and for purposes of later identification the following equivalent terms will prove helpful:

The first arch or subicular area.

The second arch or fasciolar area.

The third arch or dentate area.

The fourth arch or epithelial area.

The fifth arch or chorioidal area.

The characteristic differences in the organization of these areas are illustrated in figure 2, $S A, F A, D A, E A$ and $C A$.

Cellular differences in five arches of 16 millimeter pouch young opossum

The brain wall immediately below the lower limit of the neocortex (fig. $2 N$ ) shows marked changes in character. The germinal layer is thicker, the mantle layer is especially prominent and the marginal zone reduced to a thin stratum due to the extensive migration of mantle cells into it. The region with these features forms the subicular area (fig. $2 S A$ ). The arch which it forms extends from the base of the olfactory peduncle, over the thalamus and then downward into the lowest portion of the caudal region (fig. 1, Sl. 4, Sec. 2).

The region subjacent to the subicular area shows further changes in cellular arrangement. The germinal layer is less broad and its outer boundary more sharply defined. The mantle layer is still prominent although not so broad as in the subicular layer. The marginal layer is relatively wide. This region forms the second hippocampal arch or fasciolar area (fig. $2 F A$ ). It follows the same general direction as the subicular area from the base of the olfactory peduncle to the depths of the caudal region (fig. 1, Sl. 4, Sec. 16).

The most striking cellular alteration occurs in the third hippocampal arch or dentate area (fig. $2 D A$ ). The germinal layer is predominant in this portion of the brain wall. Cells, with deeply staining nuclei and in 
densely compact arrangement, have migrated outward to such an extent that the marginal zone is reduced to an extremely thin velum. It is difficult to distinguish those cellular differentiations which, in the subicular and fasciolar areas, characterize the mantle layer. Following the course of the hippocampal arches superimposed upon it, the dentate area extends from the olfactory peduncle to the deeper portions of the caudal region (fig. 1, Sl. 4, Sec. 29).
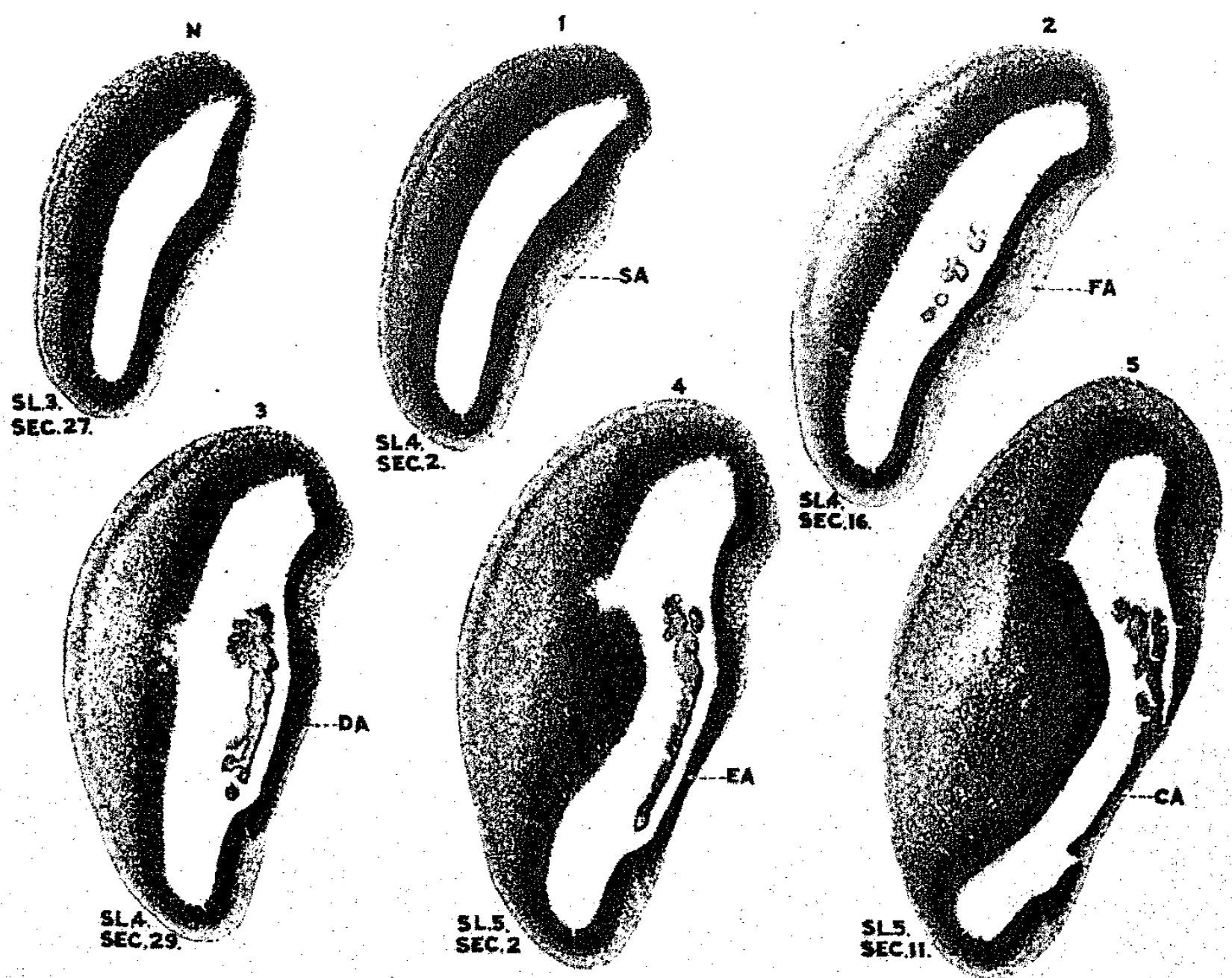

Fig. 2. Horizontal Sectrons Showing Characteristic Areas of Mestai Surface of The Left Hemisphere in 16 m. Pouch Young Opossum

Specimen No. 2005. Magnification $\times 70 . N$ (slide 3, section 27) neocortex; 1 (slide 4, section 2) subicular area (SA); 2 (slide 4, section 16) fasciolar area $(F A)$; 3 (slide 4 , section 29 ) dentate area $(D A) ; 4$ (slide 5 , section 2 ) epithelial area $(E A)$;

5 (slide 5 , section 11 ) chorioidal area $(C A)$.

The next succeeding or fourth arch does not participate directly in the formation of the archicortex. It bears important relations, however, to the development of the hippocampal commissure and the fimbria. This arch forms the epithelial area (fig. $2 E A$ ). Its thickness is about one-half that of the dentate area and its cells are arranged as stratified epithelium. Another of its distinguishing features is the absence of any marginal zone. 
In its arched course it extends from the anterior pillar of the foramen of Monro upward and backward above the formen and, after circling the lateral surface of the optic thalamus, it curves forward to terminate in the posterior pillar of the interventricular foramen (fig. 1, Sl. 5, Sec. 2).

The fifth arch or chorioidal area (fig. $2 C A$ ) serves as a membranous window which closes the space formed by the superimposed arches above it. The thickness of the brain wall is here reduced to a thin membrane. The area consists of several layers of ependymal cells reenforced by a growth of meningeal mesenchyme. This meningeal growth produces several invaginations which, protruding into the lateral ventricle as chorioidal folia, later give rise to the chorioidal plexus. The ohorioidal area is attached throughout its entire extent to the lower margin of the epithelial area (fig. 1, Sl. 5, Sec. 11).

The mesial surface of the brain wall, at this stage, is generally flat, with the exception of a shallow groove which begins at the base of the olfactory peduncle, extends upward and backward above the thalamus and descends into the caudal region. This groove indicates the position in which the Hippocampal Fissure subsequently develops (fig. 1). Its presence causes a slight bulging of the mesial brain wall into the lateral ventricle. Shallow furrows surround this intraventricular bulging and mark the inception of the Hippocampal Sulci."

\section{Five arches in the hippocampal region of the 20 millimeter pouch young opossum}

The differences characterizing the five arched areas in the hippocampal region of the $16 \mathrm{~mm}$. stage are more pronounced at $25 \mathrm{~mm}$. Primary migratory lamination from the germinal layer has formed an irregularly delimited band in the subicular area quite unlike the narrow and well defined lamina of corresponding granule cells in the neocortex immediately above it. The velum forming the marginal zone has thickened until it has attained a depth nearly twice that of this layer in the neocortex. An irregular migration of many scattered granule cells appears in this lamina of the subicular area.

In the fasciolar area primary migratory lamination has resulted in the formation of a narrow band of granule cells set off by a sharp boundary line from a broad marginal zone. The distinctness of this band as well as its characteristic narrowness furmish the reasons for calling the region the fasciolar area.

In the dentate area the disposition of the granule cells is altogether

${ }^{2}$ To avoid confusion the term sulcus is reserved for grooves in the ventricular surface of the brain wall. 
different. These cells, forming a dense, compact stratum, have advanced so far toward the outer surface of the brain wall that only an extremely narrow marginal zone or velum appears external to them.

The most important advances of this stage have occurred in the epithelial area. A well defined tract-bed has made its appearance in the base of this area along the line of its contiguity with the dentate area. In spite of the fact that its structure is definitely fibrillated, it has seemed wiser to look upon it as the bed of a tract rather than a tract, until the nature of its fibrils has been determined. The bed itself occupies the position of the fimbria. It can be traced to the tip of the temporal horn of the lateral ventricle. It follows the course of the epithelial area and extends forward into the septum. All along the base of this area, the fimbrial tract-bed appears as an ingrowth of fibrils between the ependymal lining on the ventricular side and a thin layer of epithelium on its inner surface. As it extends forward into the frontal region, it gradually increases in size and, at the junction of the epithelial area with the pars tenuis of the lamina terminalis, undergoes a partial crossing to form the fimbrial decussation. This decussation occupies a position in the lamina terminalis immediately below the paraphyseal evagination and above the pars crassa which contains the fibrils of the massive anterior commissure. By this addition of the fimbrial tract-bed, the hippocampal formation has acquired the last of its arched structures.

Figure 3 shows a reconstruction of the mesial wall of the hemisphere in the $25 \mathrm{~mm}$. pouch young opossum. The hippocampal groove is still shallow although in the frontal and temporal regions it has begun to assume considerable depth. These regional differences in the hippocampal groove make it convenient to subdivide the mesial surface of the hemisphere into certain quadrants. In the anterior guadrant, the hippocampal groove has the greatest depth. It is directed obliquely upward and backward from the base of the olfactory peduncle towarcl the vertex of the hemisphere. From this region it extends backward and passes through the superior quadrant in which it is relatively shallow. In the posterior quadrant it turns downward toward the temporal region and again acquires greater depth. In the inferior quadrant the hippocampal groove extends forward into the temporal region.

The subdivision of the mesial surface into these quadrants is especially helpful in identifying the subsequent changes which take place in the several parts of the hippocampus. A notable feature of this stage is the marked expansion which has occurred in the temporal portion of the hemisphere. This expansion is associated with the development of the descending horn of the lateral ventricle.

As most of the elements ultimately entering into the hippocampal 
formation have made their appearance at this early stage, they may be enumerated as follows:

1. The five histologically distinct arched areas of the hippocampal region (fig. 3).

2. The hippocampal groove which marks the subsequent position of the hippocampal fissure. The development of this groove is accompanied by a protrusion of the mesial brain wall into the lateral ventricle forming

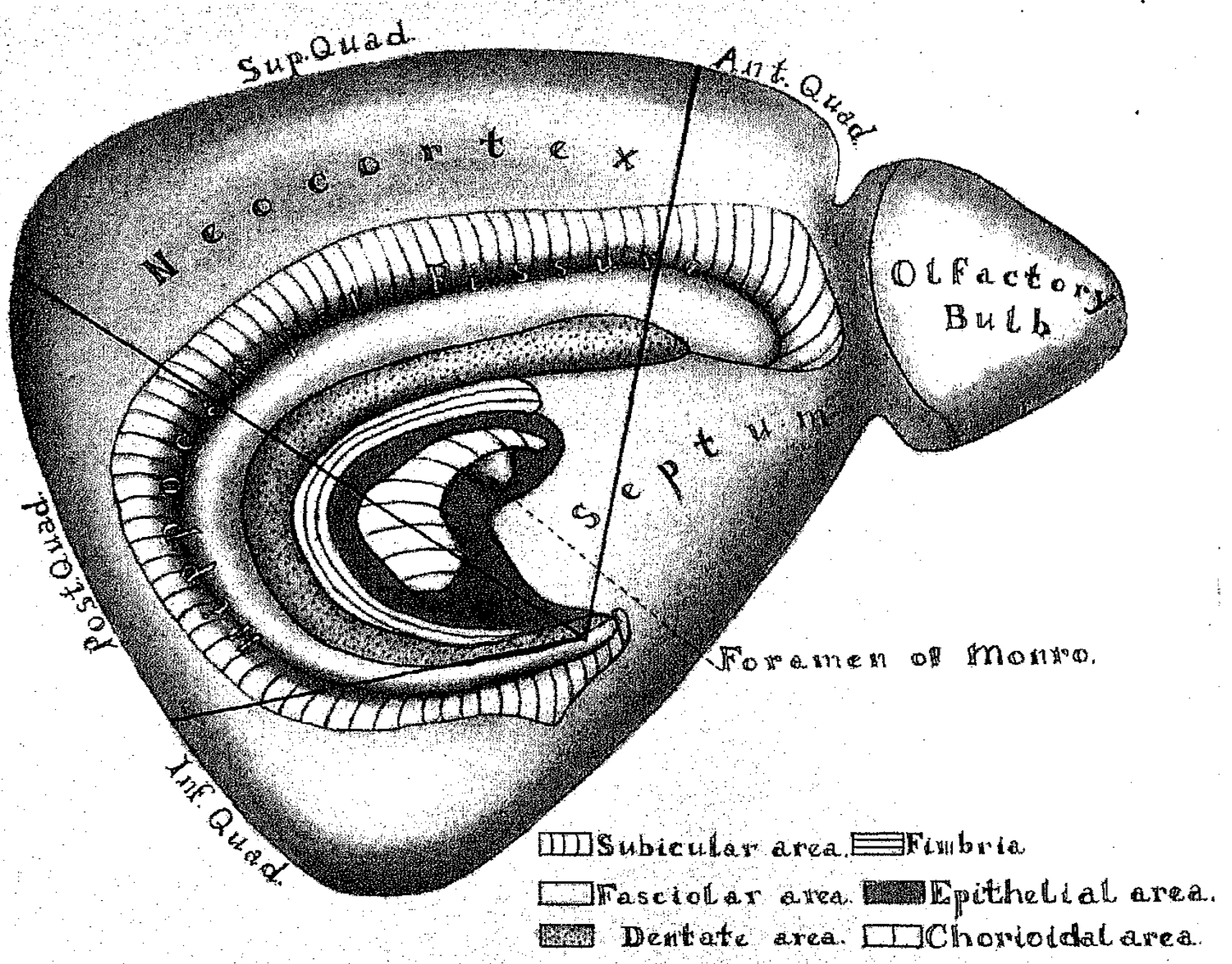

Fig. 3. Reconstruction Showing Mestal Surface of Lefp Hemisphere in 25 mm. Pouch Young Opossum

Specimen No. 2003. Magnification $\times 30$

the hippocampal eminence. A furrow on the ventricular surface, the inferior hippocampal sulcus, forms a partial boundary of this eminence.

3. The subicular, fasciolar and dentate areas participate directly in the formation of the archicortex (fig. 3).

4. The epithelial area, along its base, serves as a support for the ingrowth of the fimbrial tract-bed. By its ventral margin it gives attachment to the chorioidal area from which the chorioid plexus of the lateral ventricle is derived. Joining with the pars tenuis of the lamina terminalis 
it establishes a pathway across which the fimbrial tract-bed undergoes partial decussation, the fimbrial decussation.

Hippocampal region in 35 millimeter pouch young opossum

The $35 \mathrm{~mm}$. pouch young opossum has acquired all of the parts which enter into the hippocampus. Although these structures are relatively immature, they foreshadow the ultimate differentiations in this region of the brain. The additional features in the hippocampus at this stage comprise the hippocampal commissure and the alveus together with further specializations in the subicular, fasciolar and dentate areas (fig. 4).

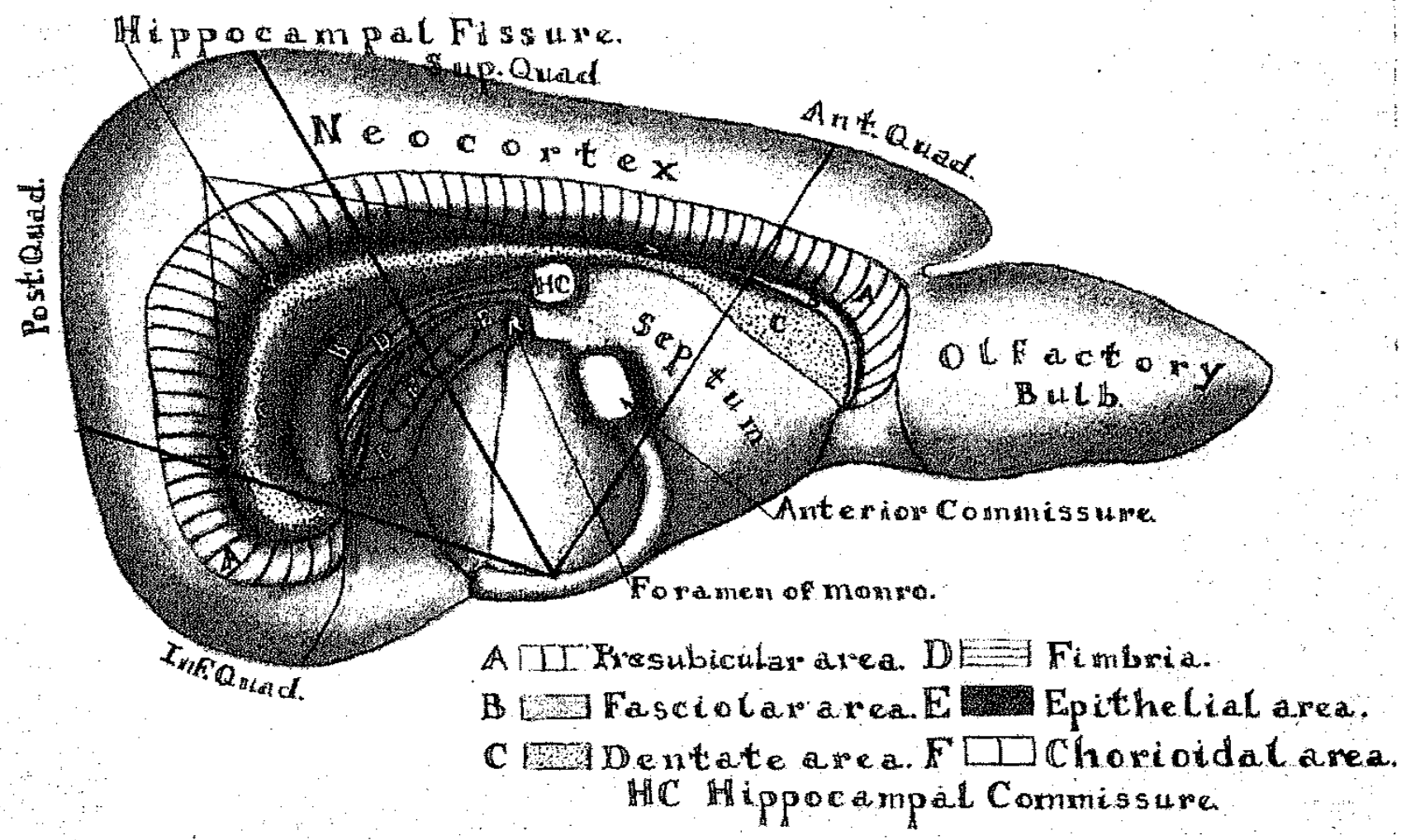

Fia. 4. Reconstruction Showing Miesial Surface of Left Hemisphere in 35 ma. POUCH YOUNG OPONSUM

Specimen No. 2004. Magnification $\times 30$

Certain of these hippocampal features show considerable variation in the several quadrants of the mesial brain-wall. Cellular conditions in the hippocampus of the anterior quadrant are shown in figure $5, A, B$, $C$ and $D$. In the most cephalic portion of this quadrant (fig. $5 A$ ) the mesial wall presents a slight undulation which forms the anterior portion of the hippocampal groove $(H)$. In this region the marked deepening of the marginal zone indicates the transition from archicortex to neocortex. The shallow hippocampal groove produces a slight bulging of the brain wall into the lateral ventricle which forms the beginning of the hippocampal eminence ( $H E)$. 

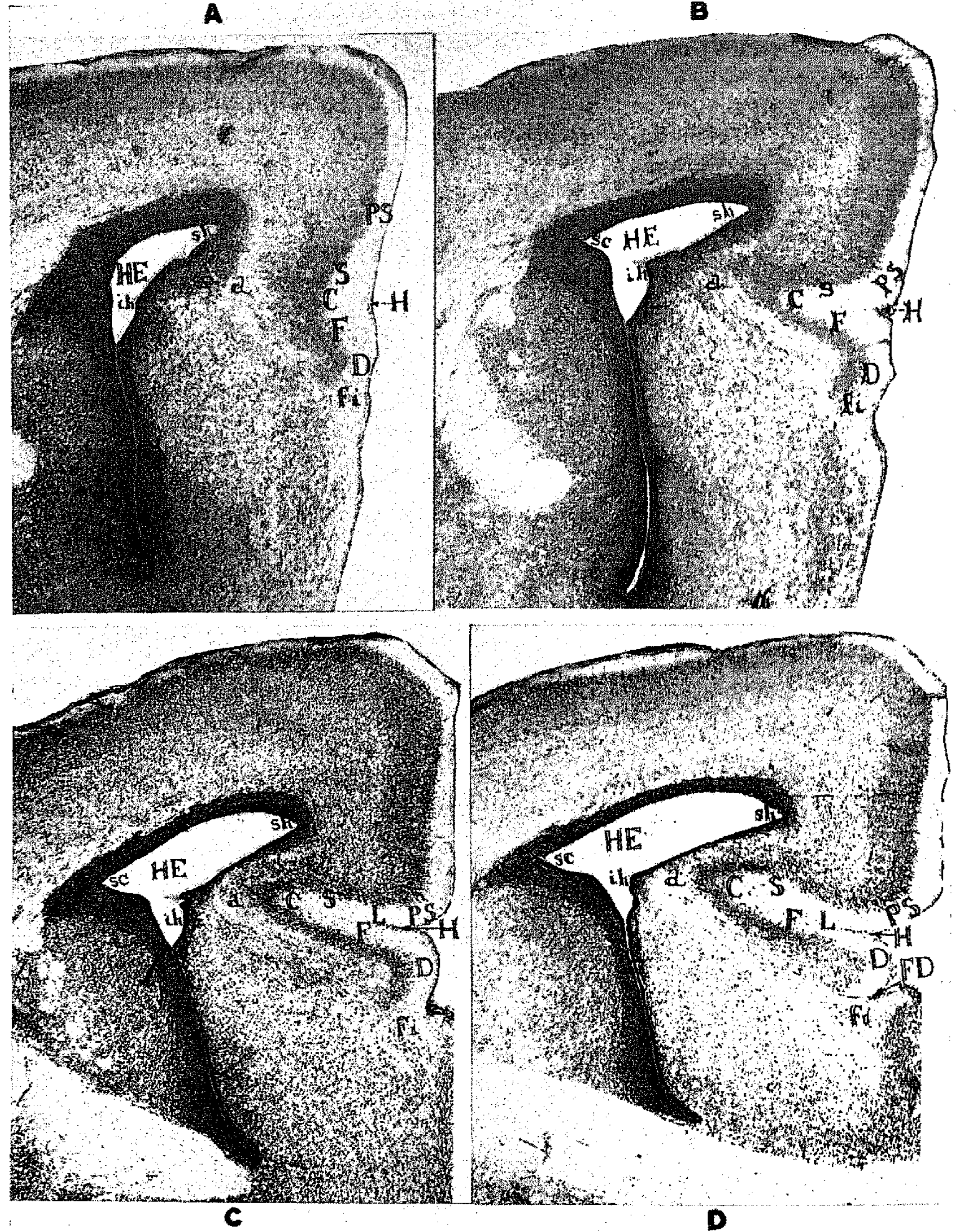

Fia. 5. Transverse Sectrong Showing Constituments of Fippocampa Formation IN ANTERIon Quadrant of 35 MM. Pouch Young Opossum

Specimen No. 2004, slide 27, section 9; slide 28 , section 16 ; slide 30 , section 3 ; slide 30 , section 37 . Magnification $\times 50$. a, alveus; $C$, crescentic area; $D$, dentate area; $F$, fasciolar area; $F D$, fimbrio-dentate fissure; $f i$, fimbrin; $I T$, hippocampal fissure; $H E$, hippocampal eminence; ih, inferior hippocampal suleus; $l$, lamina superior hippocampal sulcus. 
The most pronounced changes, however, are due to cellular specializations which have occurred in the subicular area, as a result of which this area undergoes further subdivision. $U_{p}$ to this time differentiation throughout the area has remained uniform. This homogeneity is now replaced by further local specialization.

The boundary between neocortex and archicortex, clearly indicated by the increased depth of the marginal zone, is further emphasized by a reduction in the number of granule cells in the hippocampal region of transition. Beneath the granule layer of this area are six to eight rows of small pyriform cells resting upon a compact mantle layer. This portion of the archicortex forms the presubicular area ( $P S$ in fig. 5). Adjacent to it, the cortical organization shows still more pronounced modifications. The marginal zone has increased in depth, external granule cells are almost entirely lacking and the chief elements are large pyriform cells with faintly staining cytoplasm. Because of its cortical relations this region is called the subicular area ( $S$ in fig. 5). The next succeeding portion of the archicortex, designated the crescentic area ( $C$ in fig. 5), is distinguishable by its general appearance in cross section as well as by its structural characteristics. Its marginal zone is broad, its external layer of granule cells well developed. Underlying these cells is a stratum of medium sized pyriform cells six to eight rows deep. The pyriform cells rest upon a loose and relatively narrow mantle layer. This area of the archicortex is in direct relation with the summit of the hippocampal eminence $(H E)$ and the apex of the hippocampal fissure $(H)$. The mamner in which it is eurved about the fissure causes its characteristic crescentic appearance.

Due, therefore, to further cellular specialization, the original subicular area of earlier stages becomes the presubicular area $(P S)$, the subicular area $(S)$ and the crescentic area $(C)$.

In this quadrant the fasciolar ( $F$ in fig. 5 ) and the dentate areas ( $D$ in fig. 5) complete the cortical elements of the hippocampal formation. The cells of the fasciolar area are arranged in a narrow compact layer. They consist of large pyriform elements with a few scattered granule cells. The clentate area, on the other hand, is made up largely of granule cells with irregularly scattered pyriform cells beneath them.

Another feature of this stage is the appearance of a tract-bed situated between the ependymal lining of the ventricle and the base of the archicortex. It holds the position and relations ultimately maintained by the alveus ( $a$ in fig. 5). Immediately ventral to the dentate area $(D)$ is a tract-bed representing the anterior projection of the fimbrial system $(f i$ in fig. 5).

As the hippocampal groove changes from its vertical to its horizontal course in the anterior quadrant, it rapidly deepens to form an actual fissure ( $H$ in fig. $5 B$ and $5 C$ ). With the deepening of this fissure the hippo- 
campal eminence $(H E)$ in the lateral ventricle becomes more pronounced. The hippocampal fissure, because of its increasing depth, acquires an upper and a lower lip. The upper lip consists of the presubicular and subicular areas ( $P S$ and $S$ in fig. 5). The actual depth of the hippocampal fissure is somewhat abbreviated by the development of the lamina interposita ( $L$ in fig. $5 C$ ). This lamina is an extension of the meningeal mesenchyme, which in the deeper portions of the fissure, causes a fusion between the opposite surfaces of the zonal layer covering the subicular area $(S)$ above and the fasciolar area $(F)$ below the fissure.

An undulation of the mesial surface below the hippocampal groove marks the position of the fimbrial tract-bed ( $f$ in fig. 5). As the lower lip of the fissure increases in prominence (fig. $5 B$ and $C$ ) this undulation becomes deeper and forms the fimbrio-dentate fissure ( $F D$ in fig. $5 C$ ).

In the superior quadrant, the general plane of the hippocampus is horizontal. The archicortical structures contained in it curve gently above the roof of the diencephalon and form the vault of the hippocampal arch (fig. 4). This region differs from the corresponding portion of the anterior quadrant in several important details: first, in a marked deepening of the hippocampal fissure with attendant changes in the disposition of the dentate area; second, in alterations due to the presence of the massive hippocampal commissure; and third, in relation to the interventricular canal.

The hippocampal fissure progressively increases in depth from the anterior to the posterior limits of the superior quadrant. In eonsequence the lips of the fissure become still more prominent. Most of this increased prominence is due to expansion in the dentate area which has assumed the appearance of a grooved flange forming the lower margin of the archicortex ( $D$ in fig. 6). The groove of the flange is directed toward the lateral ventricle, while its convexity gives contour to the well-rounded lower lip of the hippocampal fissure. Two factors, in addition to cortical expansion, appear to account for the changed disposition of the dentate area; namely, the increasing mass of fibers from the hippocampal commissure ( $H C$ in fig. $6, A, B, C$ and $D$ ) with the attendant deepening of the fimbrio-dentate fissure and the progressive increase in depth of the hippocampal fissure. Cumulative effects of these factors upon the relations of the dentate area become apparent with the appearance of the most cephalic fibers of the hippocampal commissure ( $H C$ in fig. $6 A$ ).

The continuity of the fasciolar and dentate areas, typical of the anterior quadrant, shows an increasing tendency toward interruption at the junction of these two archicortical areas. When this interruption finally occurs (fig. $6 C$ and $D$ ) the dentate area assumes a kidney-shaped outline, into the hilum of which the disconnected end of the fasciolar area projects (fig. $6 D$ ). Structurally the dentate area consists of a narrow marginal 
zone, a dense layer of granule cells and an irregular stratum of medium size pyriform cells. The area does not rest upon any discrete mantle
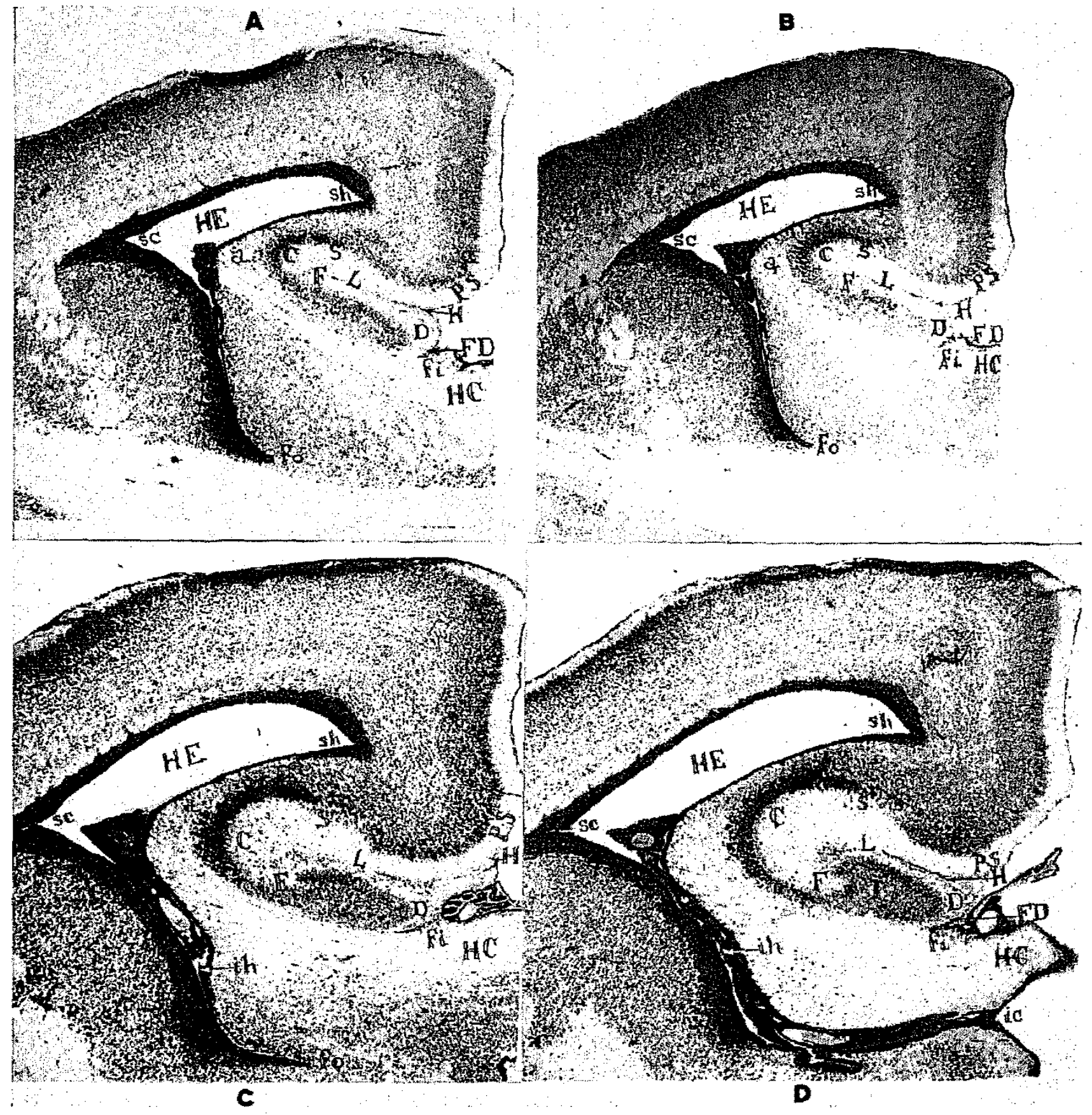

Fia. 6. Transverse Sections Showing Constituents of Hippocampal Formation in Superion Quadrant of 35 mm. Pouch Young Opossum

Specimen No. 2004, slide 31 , sections 7 and 8 ; slide 32, section 11 ; slide 33 , section 17. Magnification $\times 50$. alveus; $C$, crescentic area; $D$, dentate area; $F$, fasciolar area; $F D$, fimbrio-dentate fissure; $f$, fimbria; $f o$, foramino-olfactory sulcus; $H$, hippocampal fissure; $H C$, hippocampal commissure; $H E$, hippocampal eminence; $I$, intercalary area; $i c$, interventricular canal; $i h$, inferior hippocampal sulcus; $L$, lamina interposita; $P S$, presubicular area; $S$, subicular area; $s c$, supracaudate sulcus; $s h$, superior hippocampal sulcus.

layer because of the interposition of large fiber bundles from the hippocampal commissure. Several strands of mantle cells, however, migrating 
from the summit of the hippocampal eminence, penetrate the fiber bundles and make their way into the dentate hilum. Most of the pyriform cells of the dentate area are of medium size but at the upper pole of this kidneyshaped structure, the detached end of the fasciolar area projects into the hilum and thus introduces a considerable number of larger pyriform cells. The character, arrangement and relations of these cells give rise to a cortical region differing sufficiently both from the fasciolar and dentate areas to require special designation. Its inclusion in the dentate hilum suggests the term intercalary area ( $I$ in fig. $6 D$ ).

The other archicortical areas are essentially the same as in the anterior quadrant except that their structural details are more sharply defined. The fasciolar area ( $F$ in fig. $6, A, B, C$ and $D$ ) consists of a modorately broad marginal zone, a poorly developed granule layer, and n narrow somewhat loosely arranged stratum of large pyriform cells. Iixcept. for several strands of mantle cells, this area rests upon no definito mantlo layer. The crescentic area ( $C$ in fig. $6 A, B, C$ and $D$ ) has an extremely broad marginal zone, with a dense layer of granule and medium-sized pyriform cells resting upon a loose mantle layer. It stands out conspicuously because of its shape and the intense staining reaction of its granule cells.

The subicular area ( $S$ in fig. $6, A, B, C$ and $D$ ) is a striking feature of the archicortex, here as elsewhere. Its marginal zone is relatively broud but distinctly less than that of the crescentic area. The absence of granule cells and the clustered, almost nuclear arrangement of Iarge pyriform cells distinguish it at once from adjacent areas.

The presubicular area (PS in fig. 6, $A, B, C$ and $D$ ) consists of a mairginal zone considerably reduced in width. An ill defined granule layer and a stratum of several rows of medium-sized pyriform cells are superimposed upon a thick mantle layer. ${ }^{3}$

In the posterior quadrant, the hippocampal formation begins its descent into the temporal region, as shown in figure 7 . The most notable feature here is the increased depth of the hippocampal fissure with an attendant inrolling of the fasciolar area. As a result of this inrolling, the area fasciolaris occupies a position internal to the dentate area and in juxtaposition with the fimbria ( $F$ in fig. $7 B$ ). In the superior and anterior quadrants, the dentate area is internal to the fasciolar area. The changed relations of these two areas, caused by the increasing depth in the hippocampal fissure, has much significance. This change, in its inception, is shown in figure $7 A$. It becomes progressively more marked in figure $7 B$.

${ }^{3}$ This distinction between the six areas of the archicortex is of much importance from the phylogenetic standpoint. The recognition of these areas is likewise important in the further physiological analysis of this part of the brain. Cognizance should also be taken of them in pathological descriptions which usually speak too loosely of lesions in the Cornu Ammonis or Hippocampus. 
All of the six cortical areas of the archicortex are well clefined and may be followed through the successive alterations which result in the inrolling of the fasciolar area. The presubicular $(P S)$, subicular $(S)$, crescentic $(C)$, fasciolar $(F)$, intercalary $(I)$ and dentate $(D)$ areas present their usual characteristics in figure $7 A$. The fasciolar area has assumed its actually inrolled position in figure $7 B$, and lies internal to the dentate area. Cer-
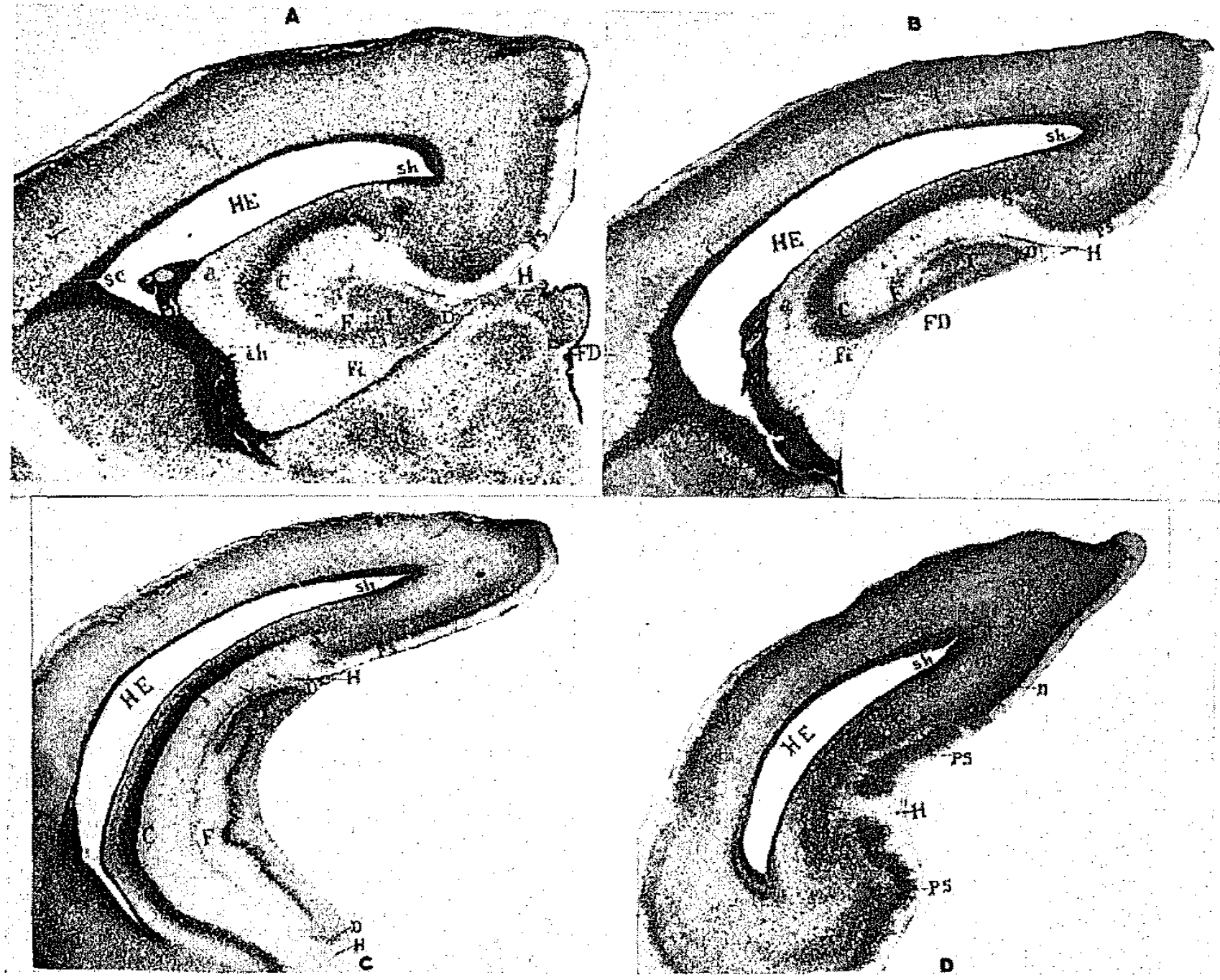

Fig. 7. Transverse Sections Showing Constitumnts of Hippocampal Formation in Posterior Quadrant of $35 \mathrm{~mm}$. Pouch Young Opossum

Specimen No. 2004, slide 35 , section 8 ; slide 38 , section 10 ; slide 40 , section 5 ; slide 43 , section 4 . Magnification $\times 50$. $a$, alveus; $C$, crescentic area; $D$, dentate area; $F$, fasciolar area; $F D$, fimbrio-dentate fissure; $f i$, fimbria; $H$, hippocampal fissure; $H E$, hippocampal eminence; $I$, intercalary area; ih, inferior hippocampal sulcus; $P S$, presubicular area; $S$, subicular area; $s c$, supracaudate sulcus; $s h$, superior hippocampal sulcus.

tain structural modifications accompany this alteration in the relations of these areas: first, an increase in the height of the lateral ventricle as it enters into the formation of the trigonum ventriculi; second, a marked expansion of the hippocampal eminence $(H E)$; and third, a lengthening of the fasciolar area $(F)$ with a deepening of the hippocampal fissure $(H)$. The specific character of the intercalary area is shown in figure $7 \mathrm{AS}$, as is also the great depth of the hippocampal fissure. 
Hippocampal region in the 45 millimeter pouch young opossum

Besides marked expansion in the neocortex, the mesial wall of the brain shows further changes in the hippocampus. In the anterior quadrant the hippocampal fissure is shallow and the archicortical areas occupy their primitive relations (fig. $8 A, B$ and $C$ ). Sections through this quadrant contain the typical succession of areas, i.e., 1 , the presubicular area; 2 , the subicular area; 3 , the crescentic area; 4 , the fasciolar area; 5 , the dentate area. The cellular features of these areas are still poorly devel-

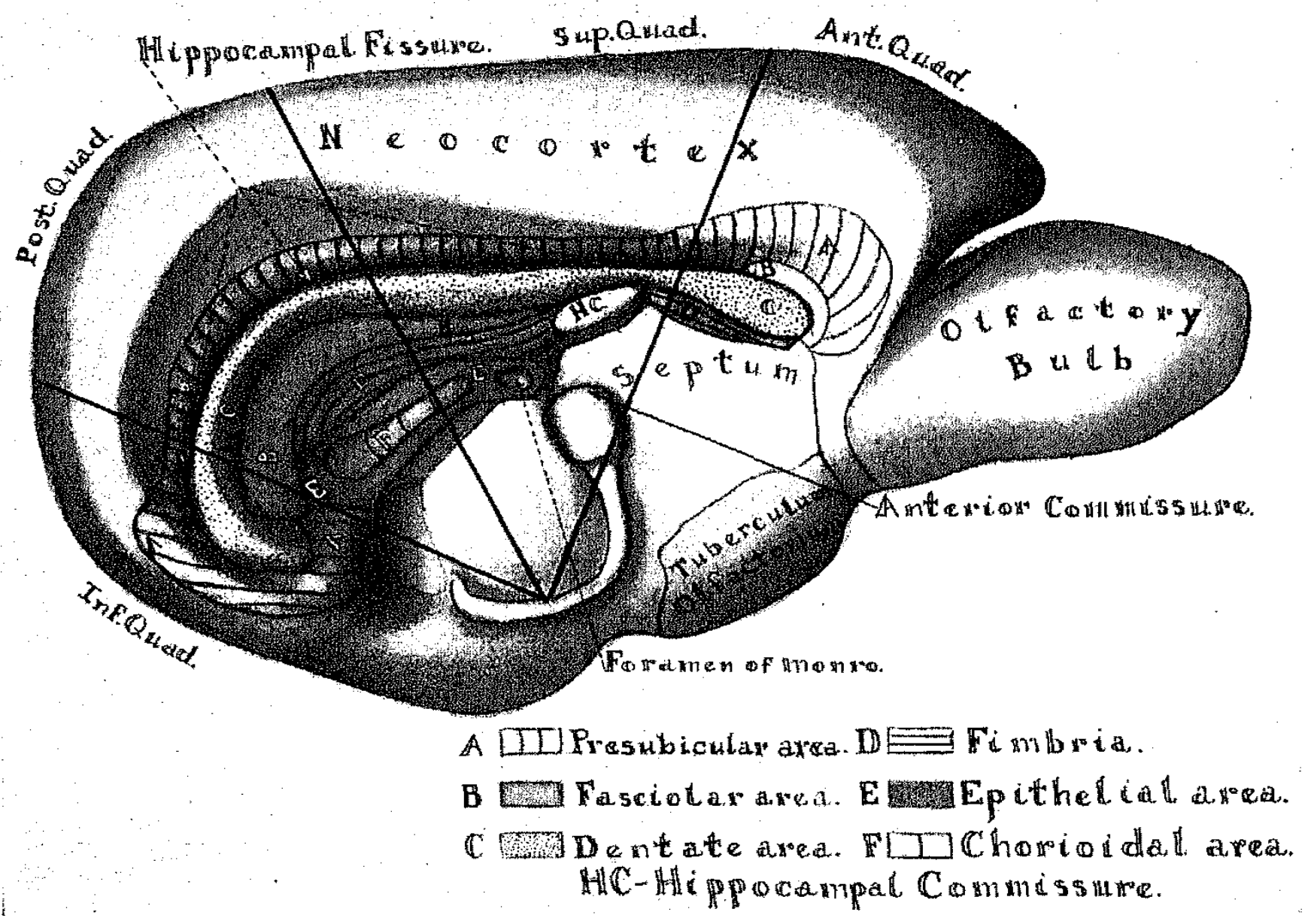

Fig. 8. Reconstruction Showing Mestal Surface of Lefr Hemispherte in 45 ma. Pouch Young Opossum.

Specimen 2066. Magnification $\times 25$

oped and lack the distinctness which characterizes them in the other quadrants. Immediately beneath the dentate area, a large bundle of fibers $(D)$ extends backward in the upper border of the septum. An extremely shallow groove forms the boundary between these fimbrial fibers and the dentate area. The groove becomes more pronounced as it approaches the superior quadrant where it assumes more definite appearance as the fimbrio-dentate fissure.

Upon passing into the superior quadrant the hippocampal fissure at once acquires greater depth, the principal effect of which is a sharp inroll- 
ing of the fasciolar area. This area now disappears from the mesial surface of the brain, being lost to sight in the deep hippocampal fissure. With the increasing depth of this fissure, the cortical inrolling continues to such an extent that the presubicular and dentate areas are the only archicortical structures to appear on the mesial surface. The results of this cortical inrolling first become apparent in the $35 \mathrm{~mm}$. pouch young opossum (fig. 7). In the $45 \mathrm{~mm}$. pouch young opossum this reversed relation of the two areas appears in the caudal portion of the superior as well as in the posterior and inferior quadrants. The altered relations incident to the cortical inrolling bring the fasciolar area $(B)$ between the dentate area $(C)$ and the fimbria $(D)$. Conditions in the anterior quadrant illustrate the primitive relations in the hippocampal region. The modifications in the other quadrants indicate the extent to which the hippocampus has departed from these earlier relations due to the increased depth of the hippocampal fissure.

At this stage the epithelial and chorioidal areas are well defined. They occupy their usual positions. The differentiation between the six cortical areas of the archicortex; ( 1 , presubicular, 2 , subicular, 3 , crescentic, 4 , fasciolar, 5 , intercalary, and 6 , dentate areas) has acquired greater distinctness.

\section{The hippocampal region in the 60 millimeter opossum}

The hippocampus in the anterior quadrant at this stage can be easily distinguished. The most cephalic portion of this archicortical region is characterized, as in earlier stages, by the extreme shallowness of the hippocampal fissure ( $H$ in fig. $9 A$ ). On the other hand, the thickness of the marginal layer as well as the bulging of the brain wall into the lateral ventricle indicate the location of the hippocampal structures.

As yet, the archicortex, with the exception of a slight undulation in the neighborhood of the hippocampal furrow $(H)$, occupies a position in the general plane of the mesial surface. With the appearance of an actual hippocampal fissure ( $H$ in fig. $9 B, C$ and $D$ ) this relation of the archicortex undergoes several changes. The hippocampus upon the surface is considerably reduced in extent and at the same time inverted toward the ventricle. As a result, the hippocampal eminence $(H E)$ becomes larger and the inferior hippocampal sulcus (ih) occupies a more inferior position in the ventricular wall. The retreat of the archicortex toward the ventricle with the deepening of the hippocampal fissure affects the dentate area last and least. The remainder of the archicortex, with the exception of the presubicular area ( $P S$ in fig. $9 A, B, C$ and $D$ ) shows the effects of inversion in a striking manner. The subicular area ( $S$ in fig. $9 A, B, C$ and $D$ ) stands out at the end of the darkly staining presubicular area as a loosely arranged nucleus. The crescentic area becomes well defined in 
the caudal portion of the anterior quadrant $(C$ in fig. $9 C)$. In this region it is also easy to distinguish the fasciolar, intercalary and dentate areas

A

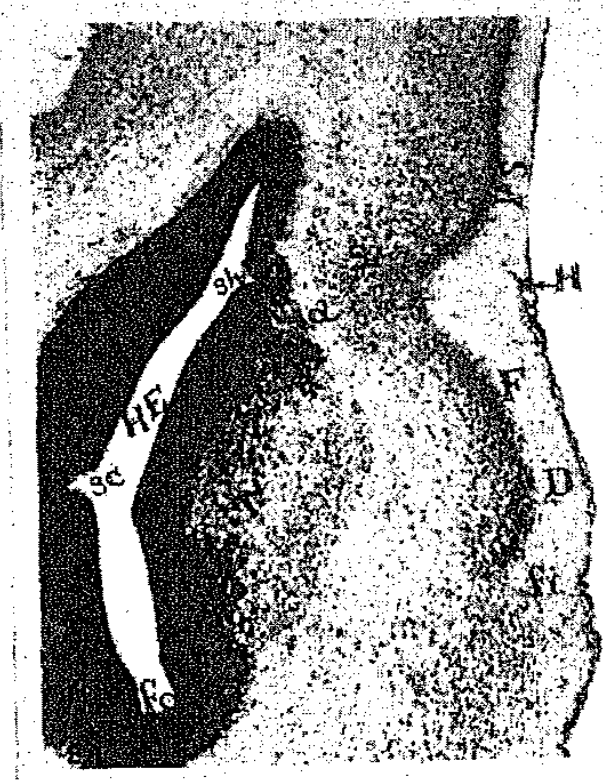

B

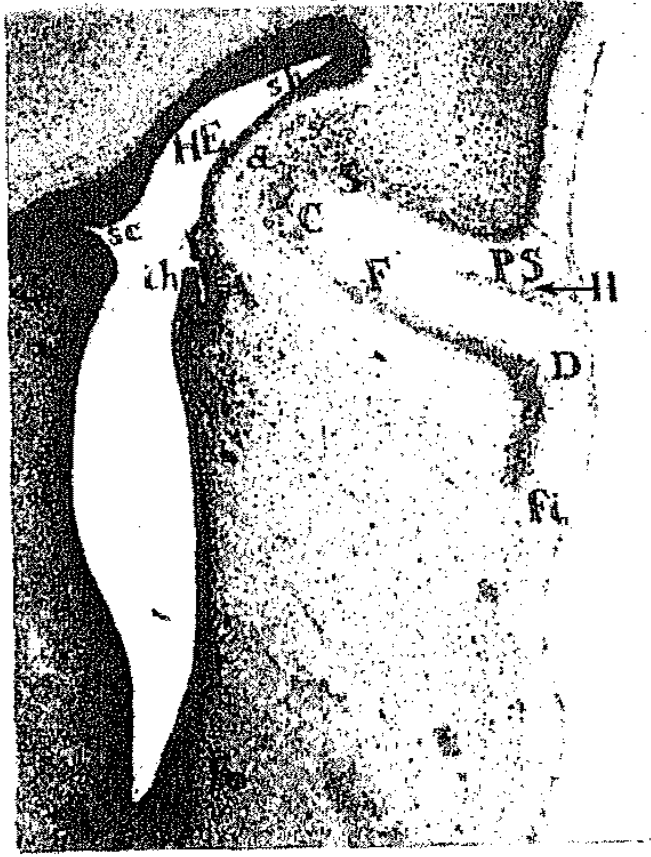

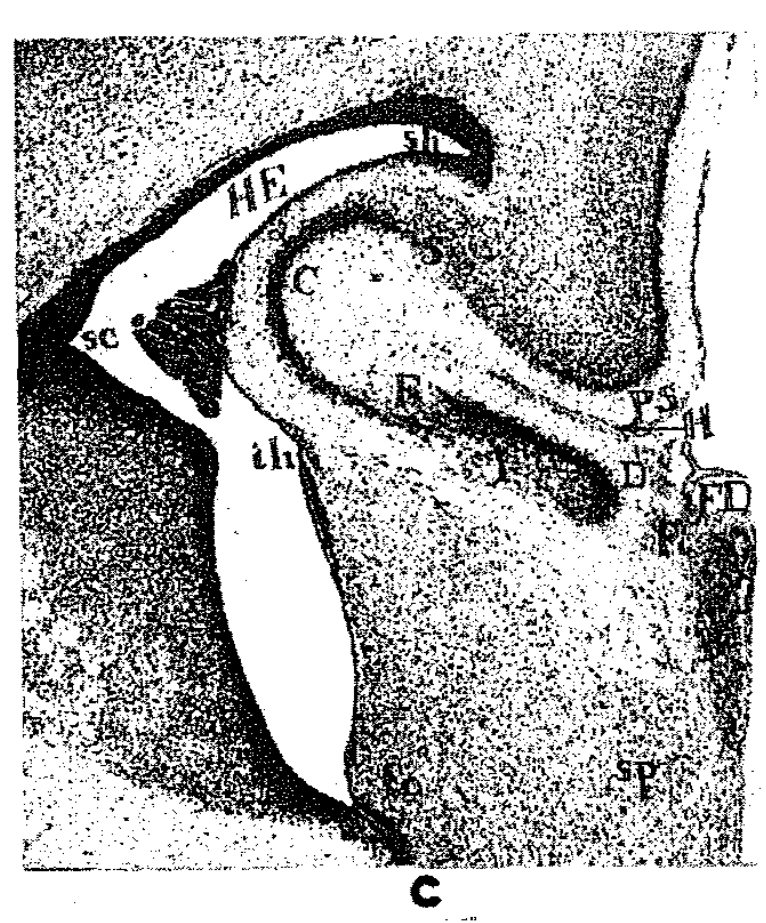

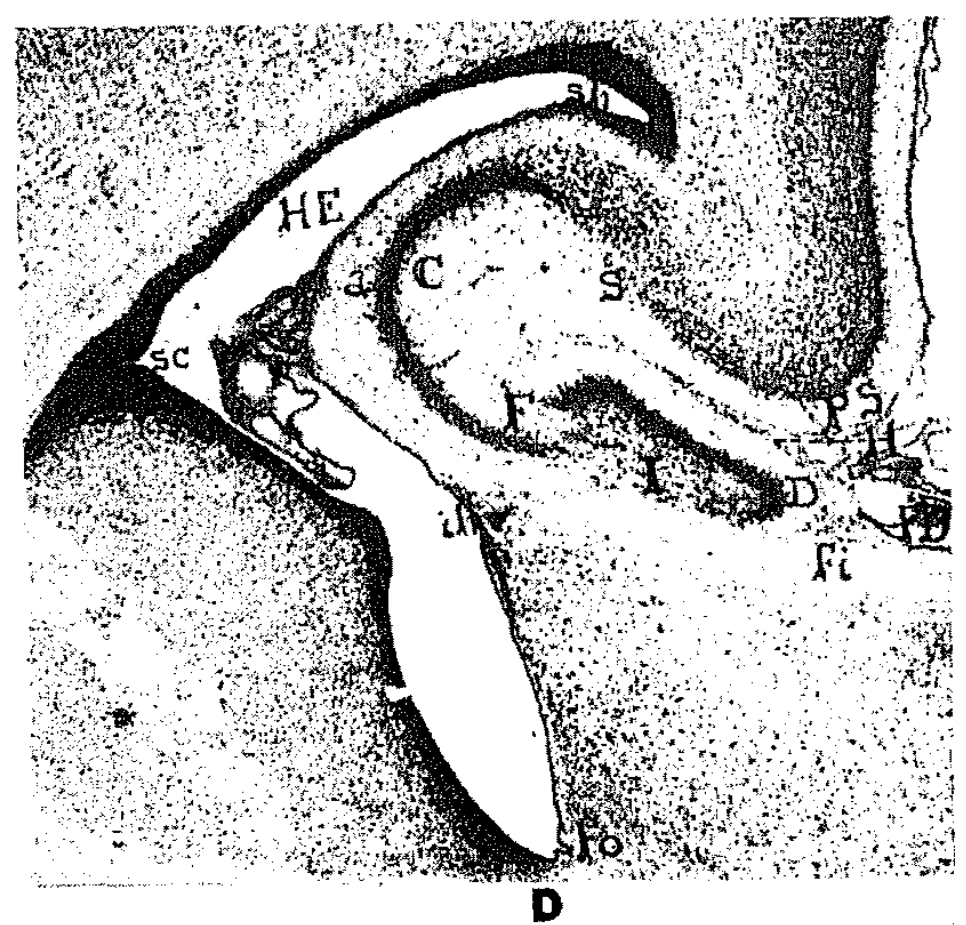

Fig. 9. Transverse Sections Showing Constritumets of Hippocamiral Fonmation in An'terior Quadrant of 60 mm. Pouch Young Orossum

Specimen No. 2019, sections $165,185,210$, and 220. Magnifieation $\times 50$. a, alveus; $C$, crescentic area; $D$, dentate area; $F$, fasciolar area; $F D$, fimbria-dentate fissure; $f i$, fimbria; $f o$, foramino-olfactory sulcus; $H$, hippoeampal fissure; $H C$, hippocampal commissure; $H E$, hippocampal eminence; $I$, intercalary area; ih, inferior hippocampal sulcus; $P S$, presubicular area; $S$, subicular area; $s c$, supracaudate sulcus; sh, superior hippocampal sulcus.

$(F, I$ and $D$ in fig. $9 C$ ). The dentate area, which originally is in direct continuity with the rest of the archicortex, has detached itself from the 
end of the fasciolar areas and is assuming the flange-like appearance characteristic of it in the other quadrants. The alveus ( $a$ in fig. $9 C$ ) is still better defined than in the more cephalic sections of this quadrant. It is prominent as an arcuate sheet of fibers which extends from the mesial tip of the dentate area and successively passes around the intercalary, fasciolar, crescentic and subicular areas. In relation to the ventricle, it occupies a subependymal position at the base of these cortical regions. Its mesial portion lies between the dentate area and the septum. In front of the inner extremity of the alveus is the fimbria, whose position on the surface of the brain is marked by the fimbrio-dentate fissure (fig. $9 C . F D$ ). Internal to the fimbria and immediately below the fimbriodentate fissure is the pars tenuis of the lamina terminalis.

The outstanding change in the superior quadrant is produced by the crossing fibers of the hippocampal commissure (fig. $10 \mathrm{~A}$ and $\mathrm{B}, \mathrm{HC}$ ). Most of the fibers pass directly to the alveus $(a)$ and thus add to the size of the hippocampal eminence. $(H E)$. This hippocampal feature in the superior quadrant furnishes one of the most striking points of contrast between mammalian brains having a corpus callosum and those without this commissure. The crossing hippocampal fibers not only increase the size of the eminence, they also increase the degree of hippocampal inrolling and give greater obliquity to the hippocampal fissure $(H)$. In the anterior quadrant the plane of this fissure is nearly horizontal; in the superior quadrant, it has an inclination of about $45^{\circ}$.

The portion of the hippocampus which appears on the mesial surface is less than in the anterior quadrant due to the further retreat of the archicortex into the ventricle as the neocortex develops. Only a part of the upper and lower lips of the archicortex surrounding the hippocampal fissure is now visible upon the inner surface of the brain. In the upper lip, the presubicular area ( $P S$ in fig. 10) can be seen on the surface; in the lower lip is the dentate area, which now has its typical flange-like appearance in cross-section ( $D$ in fig. 10). The structural features of the remaining areas of the archicortex have the same characteristics as the corresponding areas in the anterior quadrant. The subicular area is more prominent as a nuclear aggregation of pyriform cells with faintly staining cytoplasm ( $S$ in fig. 10); the crescentic area is conspicuous because of its shape and dense, darkly staining granule cells ( $C$ in fig. 10); the fasiolar area shows attenuation in size and cellular density ( $F$ in fig. 10); and the intercalary area has its distinguishing features as a portion of the archicortex inserted into the hilum of the detached, flange-shaped dentate area ( $I$ in fig. 10).

The sulci of the ventricle have likewise been modified by the changes occurring in the superior quadrant. The superior hippocampal sulcus (fig. 10, sh) has become deeper and more widely separated from the inferior hippocampal sulcus (fig. 10, ih), due to the expansion of the hippo- 
campal eminence. The inferior hippocampal sulcus becomes progressively broader and more shallow (fig. $10 A, B, C$ and $D, i \hbar$ ) as it extends backward. The foramino-olfactory sulcus (fig. $10 \mathrm{~B}, \mathrm{fo}$ ) becomes deeper and swings inward as it approaches the foramen of Monro which forms a long canal beneath the hippocampal commissure.
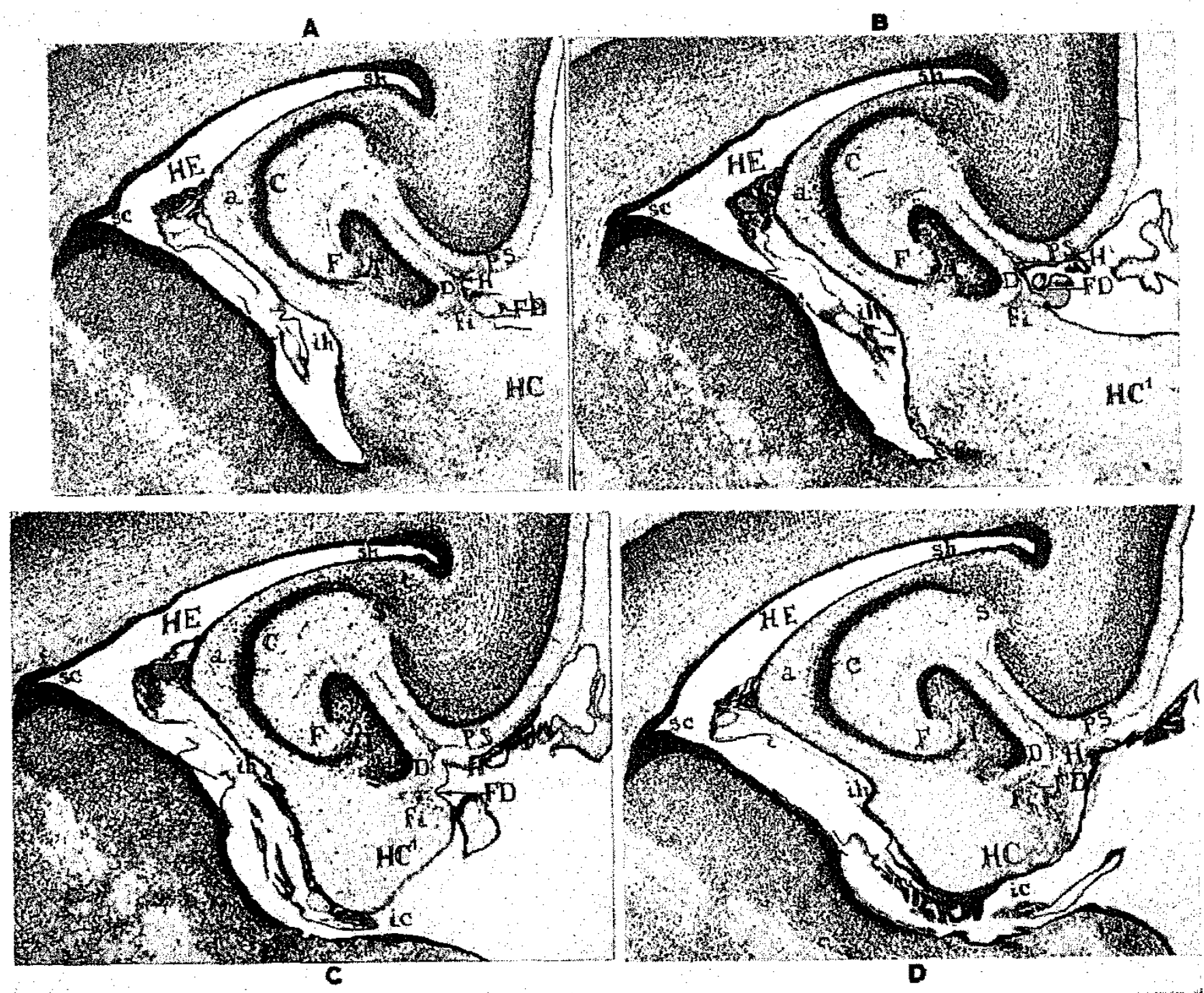

Tig. 10. Transyerse Sections Showing Constituents of Hippocampal Formation in Supterior Quadrant of 60 mm. Pouch Young Opossum

Specimen No. 2019, sections 235, 240, 250 and 255. Magnification $\times 45$. a, alveus; $C$, crescentic area; $D$, dentate area; $F$, fasciolar area; $F D$, fimbrio-dentate fissure; $f$, fimbria; $f o$, foramino-olfactory sulcus; $H$, hippocampal fissure; $H C$, hippocampal commissure; $H E$, hippocampal eminence; $I$, intercalary area; $i c$, interventricular canal; $i h$, inferior hippocampal sulcus; $P S$, presubicular area; $S$, subicular area; sc, supracaudate sulcus; sh, superior hippocampal sulcus.

The fimbrio-dentate fissure undergoes less change in the superior quadrant than the hippocampal fissure. It does, however, become less prominent as the massive bundle of crossing hippocampal fibers increases in size (fig. $10 B, F D$ ). After the commissural fibers have ceased to cross, the fimbrio-dentate fissure again becomes conspicuous (fig. $10 C, F D$ ). 
As in sections through the anterior and superior quadrants, this fiscure establishes a boundary between the dentate area and fimbria. The maintenance of this relationship has important bearings on the changed position of the fasciolar area which occurs in the posterior quadrant. The exact course of the fibers forming the hippocampal commissure cannot be determined with complete certainty in preparations made especially to give cellular detail. The process of development, however, offers a fortunate opportunity in this respect. All tract-beds and developing tracts take a brilliant white or yellow stain until myelinization is far advanced. In this way it is possible to gain a general idea of the course of many fiber bundles. Much detail of the fibers entering the hippocampal commissure is thus disclosed. These hippocampal fibers can be traced directly into the alveus. On the ventricular side they extend as far upward as the superior hippocampal sulcus. They are interposed between the ependyma of the rentricle and the successive areas forming the archicortex. After they sweep around into the region immediately beneath the dentate area, they pass through the thick mesial wall formed by the septum, enter the pars tenuis of the lamina terminalis and cross the midline in this structure. Dorsal to these crossing fibers is a smaller bundle of fimbrial fibers which undergoes decussation. Lying upon the back of these commissural and decussating fibers, is a long, evaginated portion of the third ventricle which ends in two outpouchings. The more ventral of these diverticula is the paraphyseal pouch, the more dorsal, the periphyseal pouch. ${ }^{4}$

The increased depth of the hippocampal fissure in the superior quadrant results in further inrolling of the archicortex. The cortical inrolling becomes so pronounced in the caudal region of this quadrant that the fasciolar area assumes a position on the mesial surface of the hemisphere (fig. 11). The occurrence of this cortical inrolling, with the attendant change in the relations of the dentate and fasciolar areas, has already been mentioned in discussing the $45 \mathrm{~mm}$. pouch opossum. It is a change to be borne in mind since it has particular significance in the structural relations of these areas in all mammals which develop a corpus callosum.

In the posterior quadrant the effects of the alteration in the relations of the fasciolar and dentate areas are still more striking. The change is obviously due to the sharp downward turn of the hippocampus as it descends into the temporal region. A deepening of the hippocampal fissure, as well as a lengthening of the fasciolar area, accompany this descent (fig.

${ }^{4}$ Some years ago I described the periphyseal processes in petromyzon, amia calva and ameiurus nebulosus. I am strongly inclined to believe that the dorsal periphyseal pouch in opossum is the homologue of the extensive periphyseal processes in certain fish. This question is of much interest but requires further investigation before the homology can be definitely established. 
11a). Certain contrasts in the relations of the fasciolar and dentate areas in the several quadrants are illuminating. In the cephalic region of the anterior quadrant the fasciolar area appears in the mesial surface of the brain because the hippocampal fissure is extremely shallow and no archicortical inrolling has yet occurred (fig. $9 A, F$ ). With the deepening of the fissure (fig. $9 B$ and $C, H$ ) the fasciolar area is carried inward toward the lateral ventricle and away from the mesial surface. It is carried still

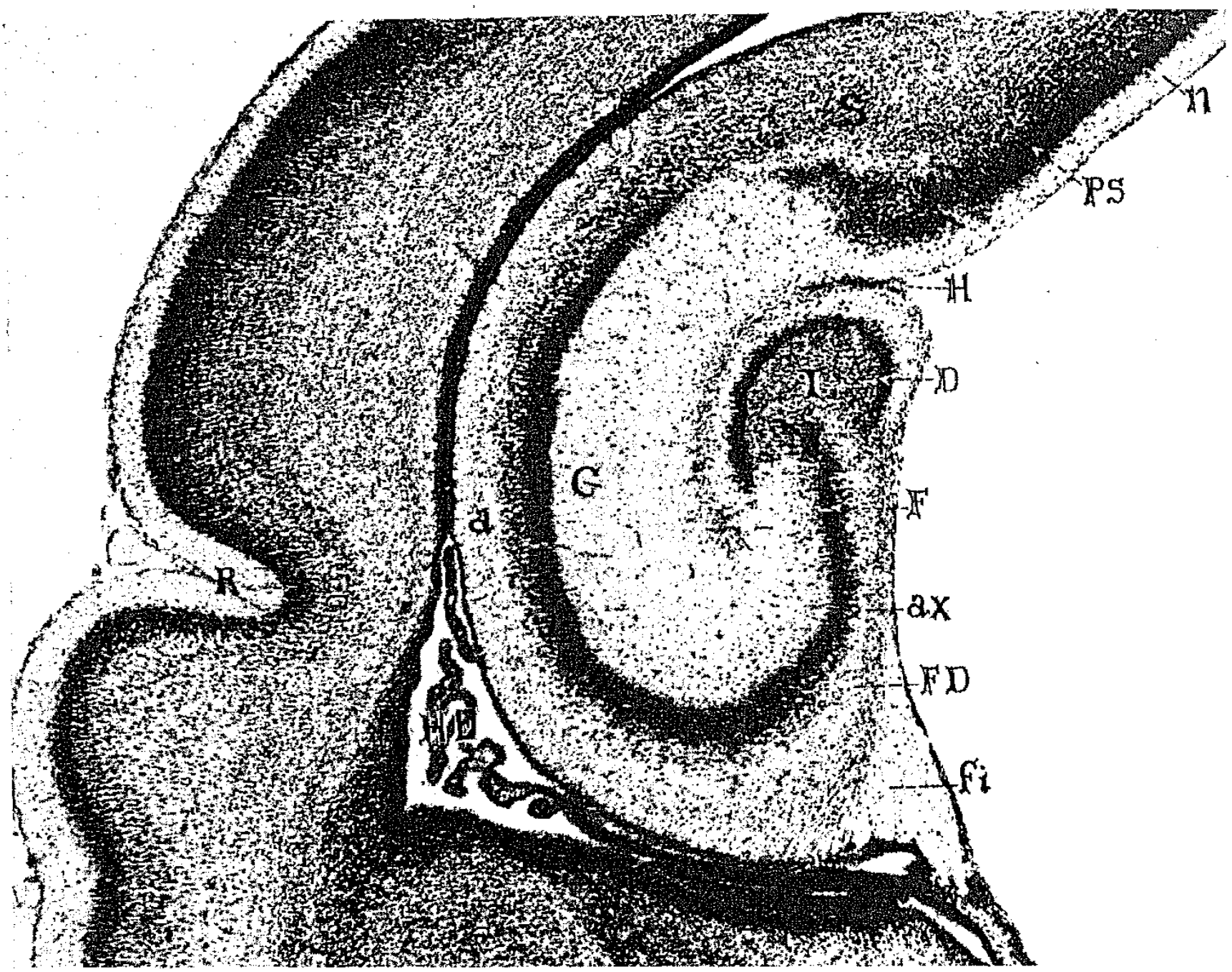

Fig. 11. Transverse Section Showing Inrolling of Fasciolat Area and ExiraVentricular Alveus in 45 ma. Pouch Young Opossum

Specimen No. 2066, section 175. Magnification $\times 60$. $a$, alveus; $a x$, extraventricular alveus; $C$, crescentic area; $D$, dentate area; $F$, fasciolar area; $F D$, fimbriodentate fissure; $f i$, fimbria; $H$, hippocampal fissure; $H E$, hippocampal eminenee; $I$, intercalary area; $n$, neocortex; $P S$, presubicular area; $R$, rhinal fissure; $S$, subicular area.

farther inward and becomes invisible upon the surface throughout the superior quadrant. Its disappearance from the mesial surface is due to the cortical inrolling incident to increased depth of the hippocampal fissure. But this same factor, i.e., the depth of the fissure which causes the fasciolar area to disappear as a feature visible upon the mesial surface, now causes it to reappear in the posterior quadrant as a prominent surface element (fig. 11, F). 
Not only does the hippocampal eminence $(H E)$ increase in size as the archicortex extends downward into the temporal region, but certain changes occur in the relation of important structures. Most conspicuous among these alterations are those affecting the alveus. In other portions of the hippocampus, this broad sheet of nerve fibers has held a char-

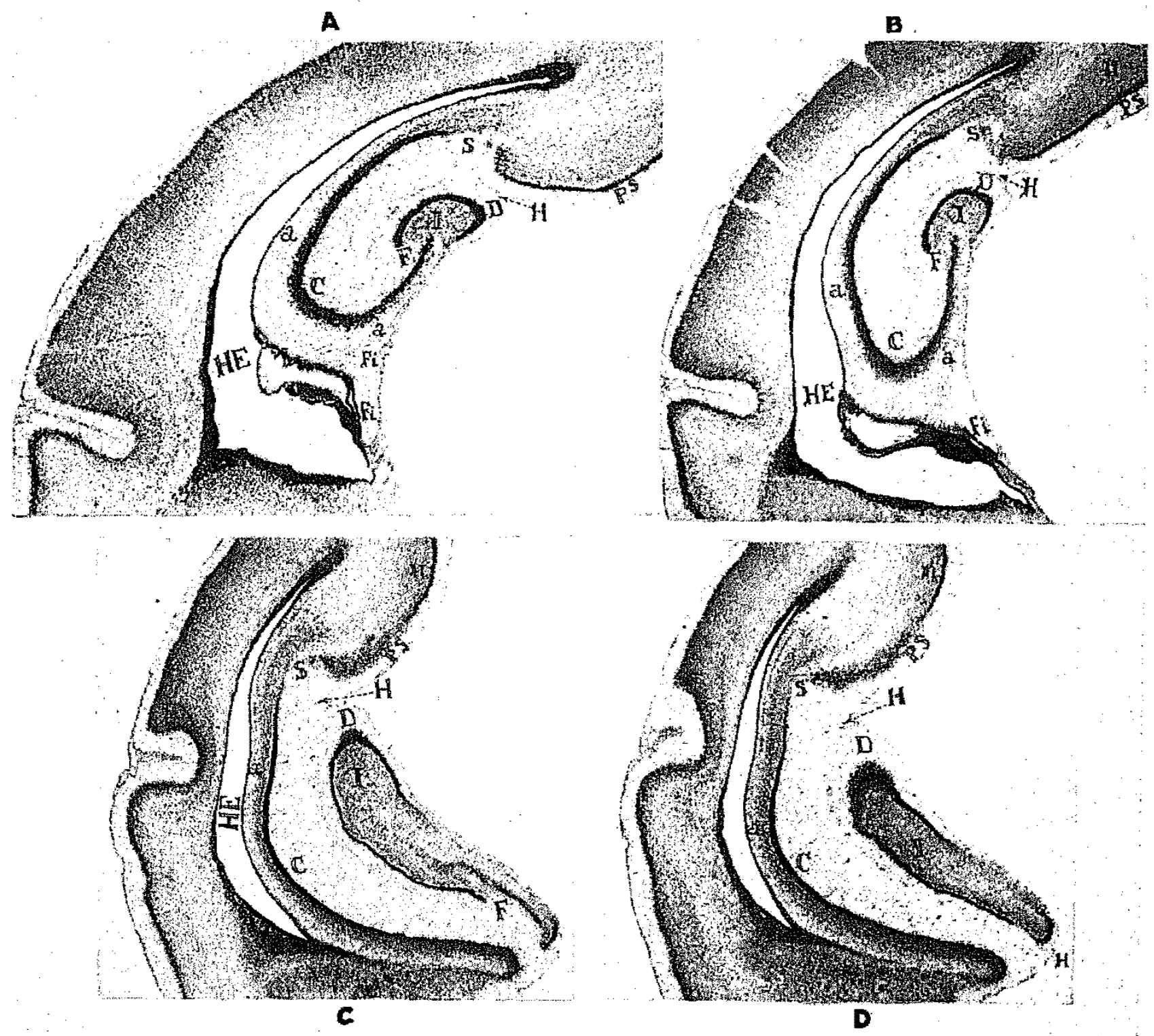

Fig. 12. Transverse Sections Showing Constituments of Hippocampal FormaTion in Posterior Quadrant of $60 \mathrm{~mm}$. Pouch Young Opossum

Specimen 2019, sections $300,320,355$, and 360. Magnification $\times 45$. a, alveus; $C$, crescentic area; $D$, dentate area; $F$, fasciolar area; $F D$, fimbrio-dentate fissure; $f i$, fimbria; $H$, hippocampal fissure; $H C$, hippocampal commissure; $H E$, hippocampal eminence; $I$, intercalary area; $n$, neocortex; $P S$, presubicular area; $S$, subicular area.

acteristic position, namely, at the base of the archicortex and immediately internal to the ependyma of the ventricle. In other words, it is an intraventricular structure (Vide $a$ in fig. $9 A, B$ and $C$; also fig. $10 A, B$ and $C$ ). Such a statement, however, requires certain qualifications. It is true as applied to the anterior quadrant and most of the superior quadrant. It 
is accurate only in part with reference to the posterior quadrant. In this region of the hippocampus the inrolling of the archicortex, already described in connection with the deepening of the hippocampal fissure, produces not only a change in the fasciola-dentate relations, but an actual eversion of the fasciolar cortex. As a result of this eversion, that portion of the alveus which underlies the fasciolar area now occupies a position in the mesial surface of the hemisphere; in other words, it has become an extraventricular part of the alveus ( $a$ in fig. $12 \mathrm{~A}$ ). During the process which results in the eversion of the fasciolar area, a marked alteration occurs in the relation of the fimbria and the dentate area. In most of its extent, this bundle of fibers has held a position in immediate juxtaposition with the dentate area. The fimbrio-dentate fissure has offered a constant surface landmark identifying the intimacy of these two hippocampal structures. But in the posterior quadrant this intimate relationship is lost. With the lengthening of the fasciolar area and the imrolling of the archicortex, the fimbria has given up its close relations with the dentate area $(f$. in fig. $12 A$ ). It has allied itself with the fasciolar area in such a way that quite a space exists between it and its former close structural associate, the area dentata. In fact, it is in this space that the extraventricular alveus makes its appearance ( $a$ in fig. $12 A$ and $B$ ).

Partly as a consequence of the elongation of the fasciolar area and partly because of the gradual alteration of the fimbria, the fimbrio-dentate fis. sure $(f d$ in fig. $12 A$ ) has become broad and shallow. The hippocampal fissure $(H)$, however, especially the lamina interposita, shows marked increase in length.

All of the archicortical areas are well defined in the posterior quadrant. The presubicular, subicular, crescentic, fasciolar intercalary and dentate areas are easily identified in the cephalic region of this quadrant. In the more caudal portions of the quadrant, the transition from archicortex to neocortex above and from archicortex to paleocortex below, may be traced without difficulty (fig. $12 \mathrm{~B}, C$ and $D$ ). The final detachment of the caudal extremity of the dentate area by the hippocampal fissure is seen in figure $12 D$.

\section{The hippocampus in the adult opossum}

The reconstruction of the adult opossum brain shows the ultimate effects of archicortical inrolling. Increase in the dimensions of the neocortex is not only a conspicuous feature of the mesial surface of the hemisphere but a general decrease in the size of the presubicular area (fig. 13) indicates the degree to which the archicortex has retreated into the hippocampal fissure.

In the anterior quadrant, the fissure is shallow with the result that the presubicular, fasciolar and dentate areas appear exposed on the surface. 
With the deepening of the fissure in the more caudal portion of this quadrant, the fasciolar area disappears and the fissure is now bounded, as in most other portions of its course, by an upper lip consisting of the presubicular area and a lower lip formed by the dentate area.

The encroachments of the neocortex upon the hippocampus are still more evident in the superior quadrant. The presubicular area is reduced in its surface expression to a narrow band of cortical tissue while the subicular, crescentic and fasciolar areas have undergone complete introversion into the greatly deepened hippocampal fissure. In the caudal portion of the fissure, the introversion is so extensive that the fasciolar area has

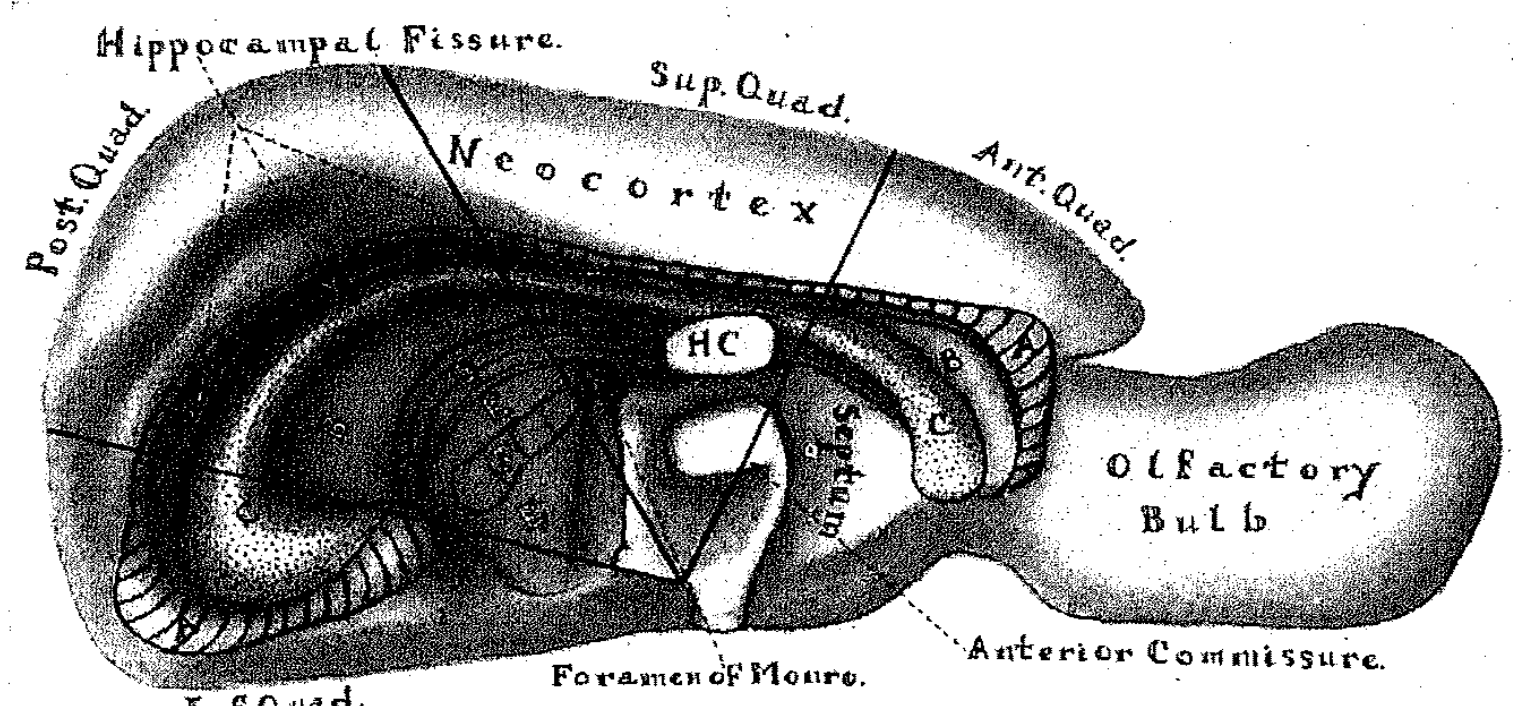

Inf.Qund.
A.EResubicular area D: TFinbria

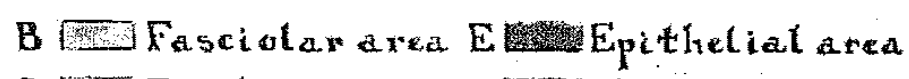

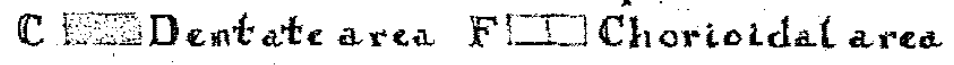 HC-Ki ippocampat Conturissure.

Fig. 13. Reconstructon Showing Mesial Surface of Left Hemisphere in an AdULT OPOSSUM

Specimen No. 2013. Magnification $\times 15$.

begun to assume a position inside of the dentate area, interpolated between the dentate and the fimbria. The epithelial and chorioidal areas occupy their usual positions.

In the posterior quadrant, the hippocampus makes its sharp descent downward into the temporal region and comes into relation with the trigonum of the lateral ventricle. Here the archicortical introversion is carried to its highest degree and the fasciolar area is most conspicuous in its position inside of the dentate area.

In the inferior quadrant, the hippocampus bends directly forward at right angles to its descending course, and extends into the temporal region to come into relation with the temporal horn of the lateral ventricle. The 

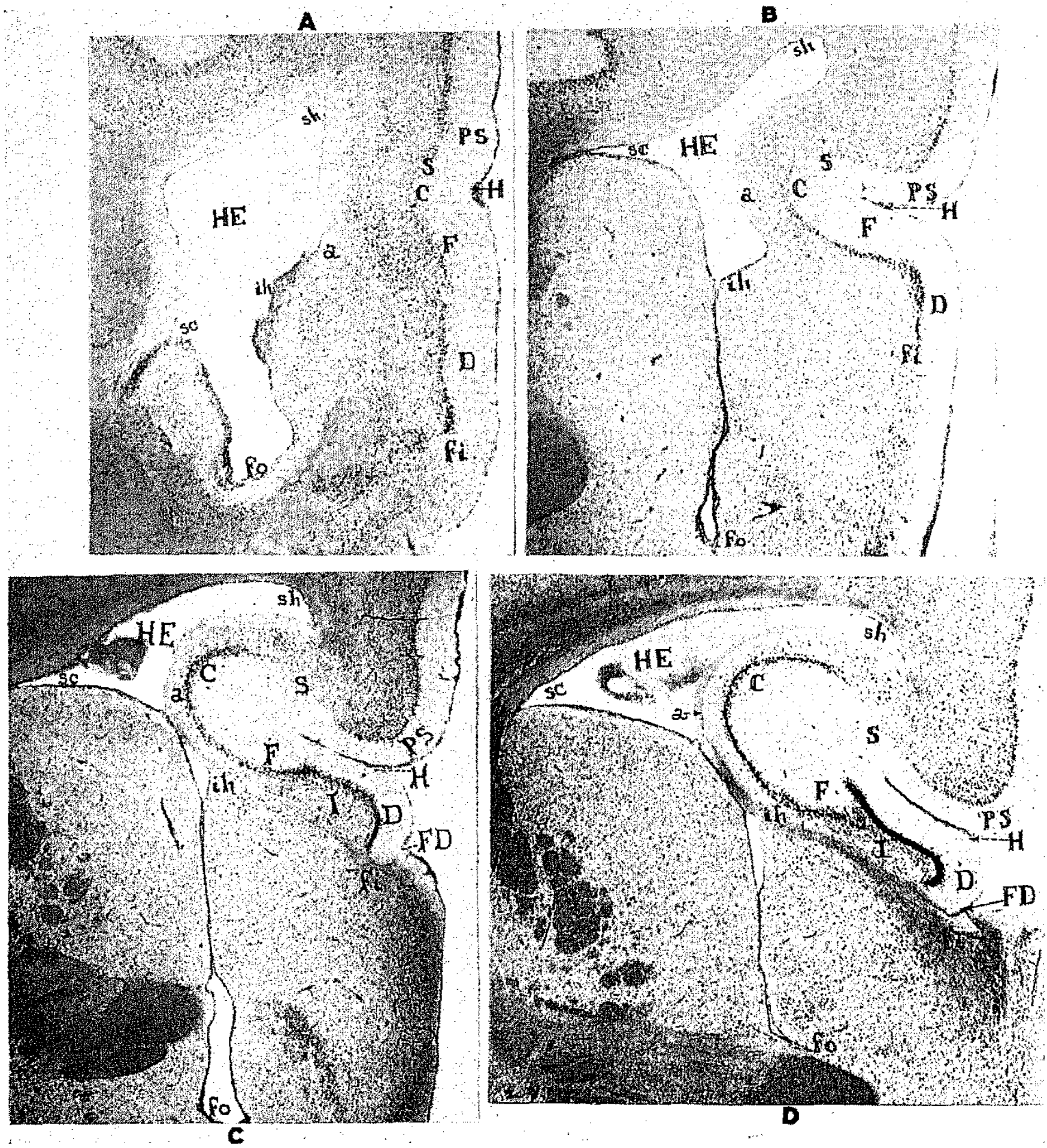

Fig. 14. Transverse Sections Showing Constituments of Hippocampal FormaTION IN ANTRRIor Quadrant OF THe ADULT OpOSSUM

Specimen No. 2013, sections 410, 450, 485, and 505. $a$, alveus; $C$, crescentic area; $D$, dentate area; $F$, fasciolar aren; $F D$, fimbrio-dentate fissure; $f$, fimbria; fo, foramino-olfactory sulcus; $H$, hippocampal fissure; $H C$, hippocampal commissure; $H E$, hippocampal eminence; $I$, intercalary area; $i c$, interventricular canal; $i h$, inferior hippocampal sulcus; $P S$, presubicular area; $S$, subicular area; $s c$, supracaudate sulcus; sh, superior hippocampal sulcus.

hippocampal fissure again assumes much of its original depth and is bounded by the presubicular and dentate areas.

Sections of the adult opossum brain at levels corresponding to those 
of the earlier stages previously discussed, show the cellular organization at maturity. In the anterior quadrant both the mesial surface of the hemisphere and the mesial wall of the lateral ventricle give evidence of the hippoeampal formation (fig. $14 A, B, C$ and $D$ ). Situated in the more cephalic region of this quadrant, the hippocampal fissure ( $H$ in fig. 14 $A$ ), appears as a shallow groove which ascends obliquely backward. Grouped around the fissure are five of the six cortical areas which characterize the hippocampus, namely, the presubicular $(P S)$, the subicular $(S)$, the crescentic $(C)$, the fasciolar $(F)$, and the dentate $(D)$. At the base of these cortical areas lies the alveus ( $a$ in fig. 14), while at the ventral extremity of the dentate area a slight undulation of the brain wall marks the beginning of the fimbrio-dentate fissure ( $F D$ in fig. $14 C$ and $D$ ).

The mesial surface of the lateral ventricle presents two elevations, the upper and smaller of which is the hippocampal eminence (HE), and the lower, the septal eminence $(S)$. The two eminences are separated by the inferior hippocampal suleus $(i h)$ while the superior hippocampal sulcus (sh) forms the upper boundary of the hippocampus.

As the hippocampal fissure turns backward into its horizontal course ( $H$ in fig. $14 B, C$ and $D$ ), the ventricular eminence of the hippocampus ( $H E$ ) increases in size and its limiting sulci become more sharply defined. Cortical inrolling proceeds rapidly and the archicortical areas acquire clearer definition. At levels marking the interruption between the fasciolar and dentate areas, the intercalary area makes its appearance ( $I$ in fig. $14 D$ ) thus completing the cortical specializations typical of the hippocampus. As in earlier stages, the hippocampal fissure increases in depth and is bounded by an upper lip containing the presubicular area and a lower lip composed of the dentate area. A comparison of figure $14 \mathrm{~A}$ and figure $14 C$ shows the manner in which the archicortex has undergone inrolling with the resulting formation of the hippocampal eminence.

In the caudal portion of the anterior quadrant the fimbrio-dentate fissure is well defined ( $F D$ in fig. $14 C$ ). Below this fissure is a bundle of fibers which forms the fimbria $(f)$. Extending from the fimbria toward the lateral ventricle is a long, narrow band of fibers, at first situated between the fasciolar area and the septum, and then lying at the base of the hippocampal eminence to form the intraventricular alveus ( $a$ in fig. $14 C$ ). The foramino-olfactory sulcus has assumed its mesial inclination as it approaches the foramen of Monro (fo in fig. $14 \mathrm{D}$ ).

The principal changes occurring in the superior quadrant are determined mainly by three factors; first, the further inrolling of the archicortex with a deepening of the hippocampal commissure and fimbrial decussation; second, the absence of the hippocampal and fimbrial decussation; and third, the junction of the foramino-olfactory sulcus with the interventricular canal. 
The decussating fibers of the fimbria as well as the commissural fibers of the hippocampus cross from one hemisphere to the other through the pars tenuis of the lamina terminalis.

It seems probable that one influence, at least, determining the marked inrolling of the archicortex in the superior quadrant is the incursion of the
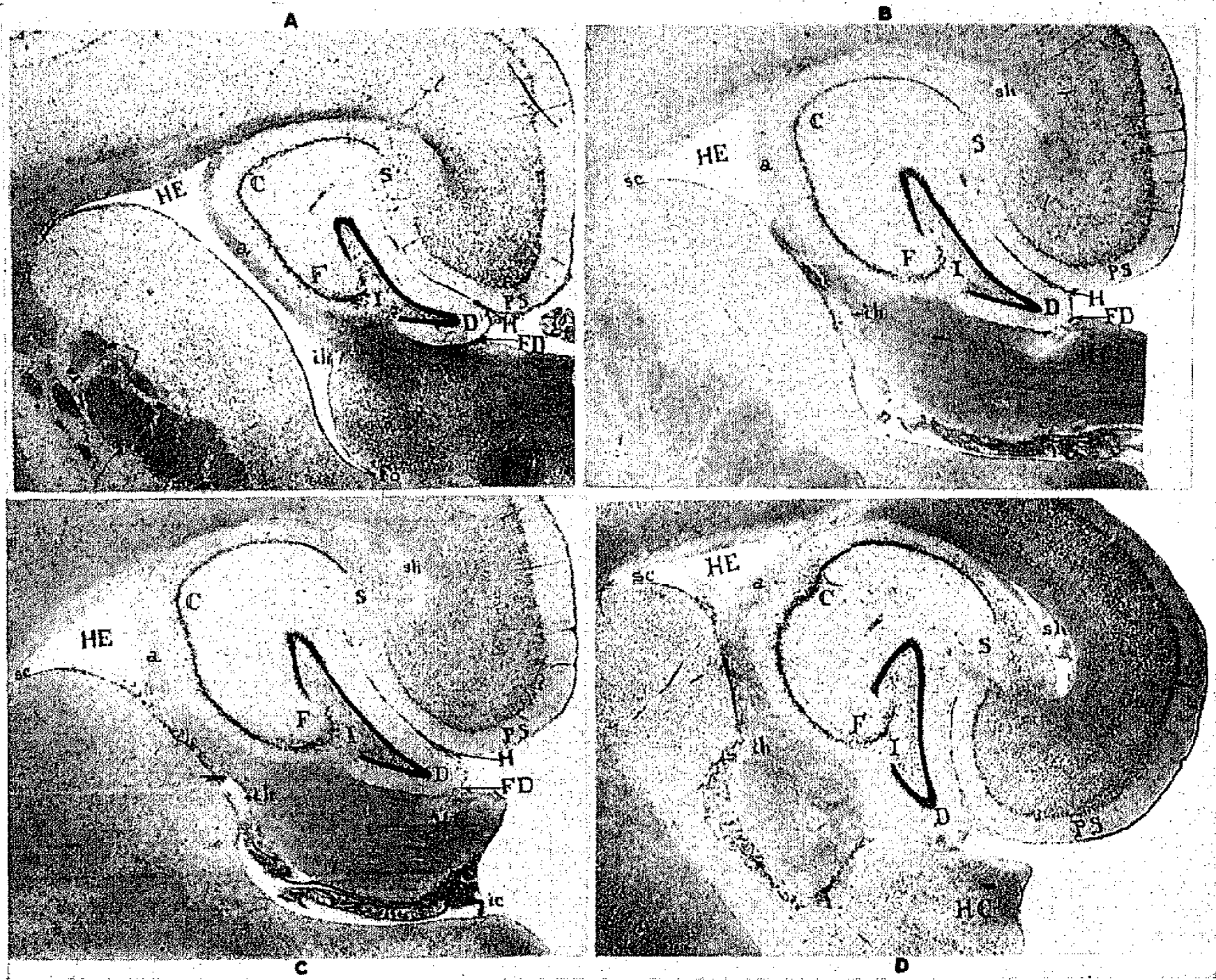

Fig. 15. Transyerse Sections Showing Constrituents of the Hippocampal ForMATION IN THE SUPERIOR QUADRANT OF THE ADULT OpOSSUM

Specimen No. 2013, sections 540,570, 580 and 600. Magnification $\times 15$. a, alveus; $C$, crescentic area; $D$, dentate area; $F$, fasciolar area; $F D$, fimbrio dentate fissure; $f$, fimbria; $f o$, foramino-olfactory sulcus; $H$, hippocampal fissure; $H C$, hippocampal commissure; $H E$, hippocampal eminence; $I$, intercalary area; $i c$, interventricular canal; ih, inferior hippocampal sulcus; $P S$, presubicular area; $S$, subicular area; $s c$, supracaudate suleus; $s h$, superior hippoeampal sulcus.

massive hippocampal commissure. Before this crossing takes place, many fibers of the fimbria pass over to the opposite hemisphere, thus forming a partial fimbrial decussation.

In the superior quadrant, cortical inrolling has advanced to such a degree that only a small portion of the archicortex is visible on the mesial 
surface of the brain (fig. $15 A, B, C$ and $D$ ). The lips of the hippocampal fissure consist of the presubicular $(P S)$ and dentate $(D)$ areas. So far has the hippocampal cortex been carried inward toward the lateral ventricle that only the inner angle of the dentate area and a small portion of the presubicular area remain exposed upon the surface.

The areas of the archicortex, already traced through the process of development, have assumed the appearances of maturity. Their underlying differences observed in earlier stages are now sublimated in adult form and clearly justify the recognition of six distinct cortical regions in the hippocampus, as follows:

1. The presubicular area is partly on the mesial surface forming the upper lip of the hippocampal fissure. Its marginal zone is a little thicker than that of the neocortex. The external granular layer is scanty, two to three cells deep. There are six to eight rows of small and medium-sized pyramidal cells. The internal granular layer is wanting. The internal pyramidal layer contains a few large pyramidal cells. A few scattered stellate cells form the multiform layer ( $P S$ in fig. $15 A, B, C$ and $D$ ).

2. The subicular area is immediately adjacent to the presubicular area. Its marginal zone is relatively thick. No granule cells are present. The entire area consists of small and medium-sized pyramidal cells having a nuclear arrangement and no evidence of stratification ( $S$ in fig. $15 A, B$, $C$ and $D$ ).

3. The crescentic area is easily distinguishable by its crescentic outline. The marginal zone is fairly deep. The external granule layer is irregular and consists of deeply staining granules and medium-sized pyramidal cells. It has no internal granule layer, and only a few large internal pyramidal cells, and a few multiform cells ( $C$ in fig. $15 A, B, C$ and $D$ ).

4. The fasciolar area has a marginal zone which is thicker than elsewhere in the archicortex. The external granule layer is composed largely of small and medium-sized pyriform cells. There are a few scattered pyramidal cells of comparatively large size but no internal granule layer and only a few multiform cells ( $F$ in fig. $15 A, B, C$ and $D$ ).

5. Intercalary area has a thick marginal zone, with some loosely scattered pyriform and granule cells. It lacks an internal granule layer, but has a number of pyriform and multiform cells in the deeper layers ( $I$ in fig. $154, B, C$ and $D$ ).

6. Dentate area, the marginal zone of which is of average thickness as compared with that of the neocortex. The characteristics of this area are the shape of the densely staining external granule layer and the configuration of the entire area. In the hilum of the dentate area there are many satellate and multiform cells. An internal granule layer is lacking and the basal region of this area is composed of multiform cells with a few pyriform elements ( $D$ in fig. $15 A, B, C$ and $D$ ). 
The plane of the hippocampal fissure in the superior quadrant has an obliquity of about $45^{\circ}$ to the mesial surface. The actual fissure itself is deep and its prolongation, the lamina interposita, adds considerably to its depth. Immediately below it is the fimbrio-dentate fissure which marks the position of the fimbria. In figure $15 \mathrm{~A}$, the fibers of the fimbrial decussation are seen passing through the upper margin of the septum

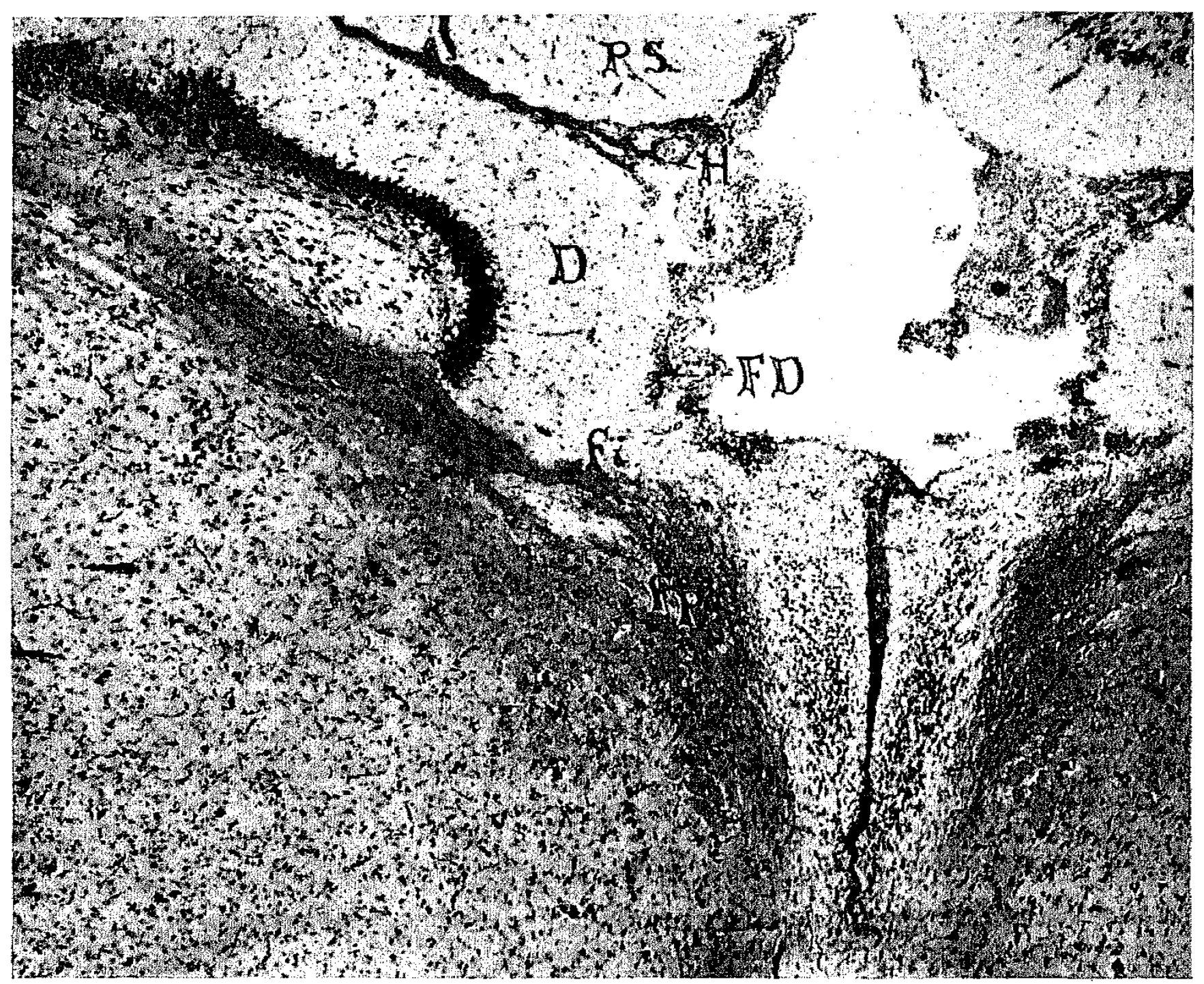

Fig. 16. Trangverse Section Showing tire So-called "Antrerior Pillat of ForNIX" In Adult Opossum

Specimen 2013, section 500. Magnification $\times 70 . D$, dentate area; $F D$, fimbriodentate fissure; $f$, fimbria; $f p$, "anterior pillar of fornix;" $H$, hippocampal fissure; $P S$, presubicular area.

whence they enter the lamina terminalis to reach the opposite hemisphere. A short clistance in front of this decussation a bundle of fibers leaves the fimbria (fig. $16 f p$ ) to descend obliquely backward to the mammillary body. This bundle, the so-called anterior pillar of the fornix, at first passes through the septum, then above and behind the anterior commissure, to complete its course to the base of the brain in the wall of the third ven- 
tricle. Since no portion of this bundle is here visible upon the mesial surface of the brain, as it is in man, there are some apparent objections to calling it the "anterior pillar of the fornix." However, its comnections and general relations leave no doubt that it is part of the fimbrial system. It would seem that the introduction of the term "fornix," to meet the needs of human anatomy, has occasioned confusion in estimating this part of the brain in its broader relations to mammals in general. The conception of a vaulted arch, or fornix, in this region, with a body supported by anterior and posterior pillars, has distinct advantages for description. On the other hand, it is not without its embarrassments, especially in such mammals as fail to present all of the characteristic parts of the arch. Such a failure occurs, as already shown, in the opossum. It also occurs in many other lower mammals. In fact it is not until the higher mammals are approached that the concept which prompted the term fornix has any real validity. More objectionable still is the lack of dynamic significance in the term which fails to call attention to the all-important feature of this bundle, namely, that it belongs to the fimbrial system of fibers in the hippocampus.

The hippocampal commissure makes its appearance in the cephalic portion of the superior quadrant. The actual crossing of the commissural fibers occurs in the pars tenuis of the lamina terminalis. The ventral aspect of this massive commissure is in relation with the long and narrow interventricular canal (ic in fig. $15 C$ ). The approach of the foraminoolfactory sulcus to the canal is shown in figure $15 \mathrm{~A}, \mathrm{fo}$. On the dorsal aspect of the hippocampal commissure and near its cephalic extremity, the fimbrial decussation crosses the midline. The main body of the commissural fibers extends outward toward the lateral ventricle to enter the alveus ( $a$ in fig. $15 A, B, C$ and $D$ ). The fimbria occupies a position along the dorsal aspect of the hippocampal commissure and immediately ventrolateral to the fimbrio-dentate fissure ( $F D$ in fig. $15 A, B, C$ and $D$ ). At the caudal extremity of the commissure, where the hippocampal fibers have ceased to cross, a massive bundle is formed which ovcrlies the interventricular canal; it consists of fimbrial and commissural fibers ( $H C$ in fig. 15C).

The alveus appears as the direct continuation of the commissural fibers. It extends over the entire ventricular surface of the hippocampal eminence ( $H E$ in fig. 15) and is covered by the ependyma of the lateral ventricle. The sulci in this ventricle are all clearly clefined (fig. 15). The superior hippocampal $(s h)$ and the supracaudate $(s c)$ sulci are much deeper than in the anterior quadrant because of the increase in size of the hippocampal eminence and caudate nucleus. The foramino-olfactory sulcus turns inward beneath the hippocampal commissure to join the interventricular canal (ic in fig. 15C). 
In the posterior quadrant, the hippocampus makes a turn downward almost at right angles to its course in the superior quadrant with the result that the hippocampal eminence ( $H E$ in fig. $17 A$ ) is much elongated. The six archicortical areas retain the same relative positions, although the crescentic and fasciolar areas have become considerably lengthened. Ac-
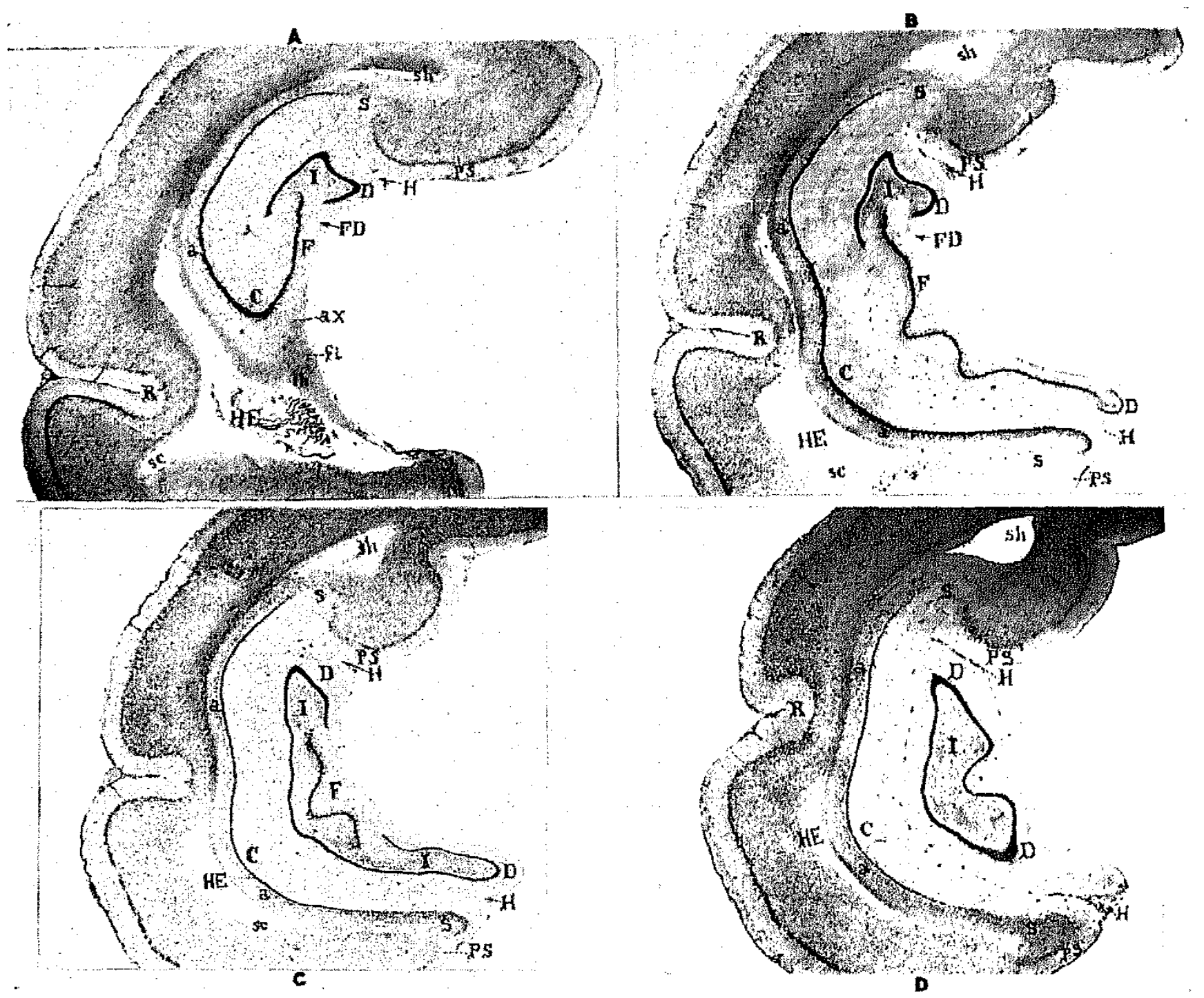

Frg. 17. Transyerse Sectons Showing Gonstituents of Hippocampal Formation in the Posterior Quadrant of the Aduli Oposingm

Specimen No. 2013, sections 705, 755, 780 and 800 . Magnification $\times 20$. a, alveus; $a x$, extraventricular alveus; $C$, crescentic area; $D$, dentate area; $F$, fasciolar area; $F D$, fimbrio-dentate fissure; $f$, fimbria; $H$, hippocampal fissure; $H E$, hippocampal eminence; $I$, intercalary area; ih, inferior hippocampal sulcus; $P S$, presubicular area; $R$, rhinal fissure; $S$, subicular area; $s c$, supracaudate sulcus; sh, superior hippocampal sulcus.

companying this surface elongation, the hippocampal fissure ( $H$ in fig. $17 \mathrm{~A}$ ) has increased in depth and caused a greater inrolling of the archicortex. The results of this inrolling make the last statement seem paradoxical inasmuch as part of the fasciolar area has assumed a position entirely outside of the lateral ventricle. It has, in fact, been overted so 
far out of its former intraventricular position that it now forms an actual part of the mesial surface of the hemisphere ( $F$ in fig. 17A). Going along with the fasciolar intraversion, the alveus connected with this area has assumed an extraventricular position which accounts for the extraventricular alveus ( $a$ in fig. 17A). The fimbria also has altered its relations. The fasciolar area is now interposed between the clentate area and fimbria ( $f$ in fig. 17A).

As the caudal extremity of the brain is approached the hippocampal formation makes a sharp bend downward which brings it into relation with the trigonum of the lateral ventricle. It then sweeps forward into the temporal region. The plane of section, now at right angles to the sections of the superior quadrant, shows all of the constituent areas and parts of the hippocampus cut lengthwise. Thus, in consequence of the deepening of the hippocampal fissure ( $H$ in fig. $17 B, C$ and $D$ ), the fasciolar area $(F)$ occupies a position internal to the area dentata $(D)$. The hippocampal fissure extends from the upper to the lower limit of the dentate area and appears to separate this region from the rest of the hippocampus. Along the upper margin of the archicortex the presubicular area (PS) is continuous with the neocortex while it adjoins the subicular area $(S)$ in the hippocampal fissure. The lower margin of the archicortex is continuous with the paleocortex whose lateral boundary, the rhinal fissure $(R)$, marks the transition to neocortex. Along this lower margin of the hippocampus the regular succession of archicortical areas assumes its usual arrangement in relation to the hippocampal fissure, the dentate area $(D)$ forming one lip, the presubicular $(P S)$ the other. The subicular area $(S)$ occupies its characteristic position, both in the posterior and inferior quadrants, situated between the crescentic $(C)$ and presubicular areas. As the hippocampus descends in its relation to the trigonum ventriculi, the hippocampal eminence $(H E)$, covered by the alveus $(a)$, assumes its greatest proportions.

IV. NATURE AND CHARACTERISTICS OF THE HIPPOCAMPUS IN THE MARSUPIAL BRAIN

The hippocampus in the marsupial brain, such as that of the opossum, possesses certain features which easily distinguish it.

In the first place, it is unitary in character, i.e., its cortical makeup and general constituency is the same throughout its entire extent, regardless of the position it may occupy, whether in anterior, superior, posterior or inferior quadrant.

In whatever region it may be viewed, the hippocampus consists of a succession of identical areas through the middle of which passes a longitudinal groove, the hippocampal fissure. From beginning to end, this fissure is bounded by the same archicortical areas and the entire hippo- 
campus is so moulded that it forms four major flexures, namely, the anterior flexure, the superior flexure, the posterior flexure and the inferior flexure. To call these several flexures convolutions or gyri serves no good purpose, since all of them are identical in their composition and are thus merely flexures of one and the same tissue plane. This fact would become

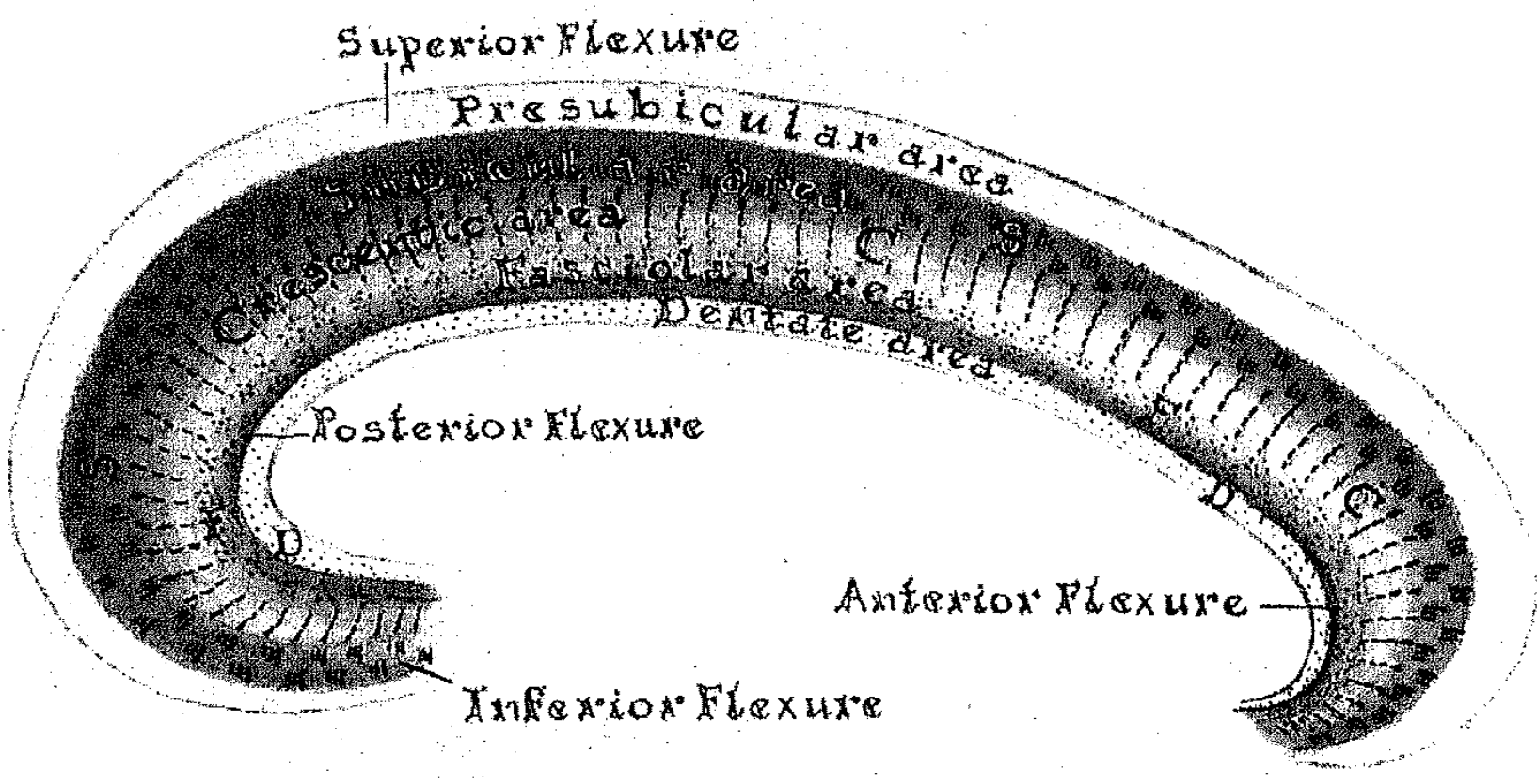

FIG. 18. Diagrammatic reconstruction of the hippocampus of the opossum showing the unitary nature of the archicortex which is made up of a series of six superimposed arched areas, namely, the presubicular, the subicular, the crescentic, the fasciolar, the intercalary and the dentate areas. $C$, crescentic area; $D$, dentate area; $F$, fasciolar area; $P S$, presubicular area; $S$, subicular area.

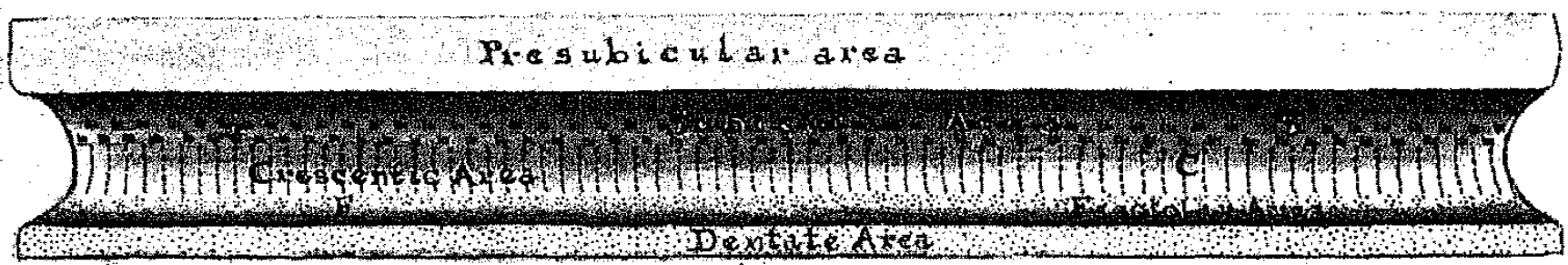

Fig. 19. Diagrammatic Reconstruction of Stretched OUt Hippocampus in the Opossum

The several flexures have been eliminated by the stretching but the succession of archicortical areas is retained in their general relation to the archicortical groove which forms the hippocampal fissure. The intercalary area is not shown in the diagrams in figures 18 and 19.

obvious were it possible to stretch out the hippocampus between its two ends and at the same time to flatten it so that the hippocampal fissure no longer formed a deep groove dividing the archicortex into an upper and a lower division (figs. 18 and 19).

In this light the unitary structural nature of the hippocampus becomes clear. The futility of designating each flexure of such a unit-structure 
as a convolution or gyrus, with all the specificity which such terms imply, is equally apparent.

The following succession of archicortical areas forms the hippocampus in the adult opossum:
1. The presubicular area
4. The fasciolar area
2. The subicular area
3. The crescentic area
5. The intercalary area
6. The dentate area

Along the inner margin of the hippocampus runs a longitudinal bundle of nerve fibers, the fimbria. All of these structures in their earliest form, as they appear, for example in the reptilian brain, are spread out in such a way as to occupy the mesial surface of the hemisphere and even to extend outward over the vertex of the brain. In the mammal the entire hippocampal formation retreats before the rapid expansion of the neocortex with the resultant formation of the hippocampal fissure and the protrusion of a large elevation of the mesial brain wall into the lateral ventricle, i.e., the hippocampal eminence.

Such are the relatively simple conditions of the hippocampus in the adult opossum and other marsupials like the kangaroo. How are these conditions affected when the corpus callosum interpolates itself into the midst of these hippocampal structures, as it does in all higher mammals?

\section{INTRODUCTION OF THE CORPUS CALLOSUM}

Before this question can be approached, it is necessary to make certain enquiries concerning the corpus callosum. For example, how does this structure establish itself as the predominant interhemispheral commissure? What is the origin of its commissural fibers? What means do these fibers employ in crossing from one side of the brain to the other? From what point of the mesial wall do they emerge? Between what structures of this wall do they penetrate? Do all of the callosal fibers make their appearance at the same time, or, if at different times, which are earliest and which latest to appear? These are questions which have been much debated and concerning which several differing opinions have been expressed. Nothing approaching unanimity of viewpoint has yet been reached. The present discussion offers the interpretation which is the result of this study. Its relation to other points of view will be discussed later.

In arriving at any tenable conclusion concerning the corpus callosum, it must be admitted at once that all mammals possessing this structure pass through antecedent stages of development. These stages follow closely those of the early brain-growth in marsupials. It may, therefore, be said that there is a precallosal stage and a callosal stage.

The precallosal conditions are well illustrated in the $31 \mathrm{~mm}$. embryo of the domestic pig (fig. 20). Horizontal sections through such a brain show the transition from the neocortex $(N)$, to the subicular area ( $S A$ in 1 ) 


$$
\theta_{0}^{8} \theta
$$


a deep groove traversing the anterior, superior, posterior and inferior quadrants of the mesial surface. It is deepest in the superior and inferior quadrants but of sufficient depth in all regions to produce a more or less prominent hippocampal eminence projecting into the lateral ventricle. The hippocampal commissure is already well developed. It begins in front of the foramen of Monro, ends immediately above it and has a cephalocaudal length of 1020 micra. The fibers of the commissure make their crossing through the pars tenuis of the lamina terminalis. The fibers of the fimbrial decussation lie below the hippocampal commissure and also pass through the pars tenuis. The fimbria is well defined especially in the anterior and superior quadrants. Its fibers pass longitudinally through the base of the epithelial area until they reach the lamina terminalis where some of them undergo decussation while the remainder extend downward in the septum forming what is generally called the "anterior pillar of the fornix." The position of the hippocampal commissure in close proximity to the foramen of Monro assumes much significance in view of later changes occasioned by the appearance and growth of the corpus callosum.

At this stage in the development of the pig's brain, all of the archipallial areas in the hippocampus may be iclentified. The transition between the neocortex and the presubicular area, although not so well defined as it becomes later, may be clearly discerned. The subicular area is easily distinguishable in all quadrants by its peculiar "nuclear arrangement". The crescentic area has become more pronounced than in earlier stages because of the increased depth of the hippocampal fissure. Both the fasciolar and dentate areas are well differentiated; it is especially to be noted that their most pronounced development has taken place in the superior quadrant which is later most extensively involved by the ingrowth of the corpus callosum. The alveus is fairly well defined and occupies its characteristic subependymal position covering the hippocampal eminence. In consequence of the general curvatures in the mesial and lateral surfaces of the hemisphere due to the rapid growth of the endbrain, the hippocampus has undergone flexion at several points so that it is convenient to speak of an anterior hippocampal flexure, a superior flexure, a posterior flexure and an inferior flexure. All of these flexures may be identified in the adult marsupial brain. In each instance they have the same significance. They indicate that the hippocampus, having a unitary tissue character throughout its entire extent is incidentally flexed at several points to form certain flexures. In animals which develop a corpus calIosum these flexures increase in number and complexity but the underlying unitary character of the tissues and structures entering into the hippocampus remain essentially unchanged. 


\section{Early embryonic appearance of the corpus callosum}

In the $83 \mathrm{~mm}$. pig embryo the earliest portion of corpus callosum to develop has made its appearance. The crossing callosal fibers are confined exclusively to the anterior quadrant and are situated in the frontal region in front of the anterior commissure. The first parts of the corpus callosum to develop are therefore the cephalic portion of the truncus, the genu and the rostrum. This statement applies equally to all other mammals embryologically examined, including the rat, guinea pig, cat and man. The relations of the several structures are best appreciated in cross-sections.

At the cephalic margin of the anterior commissure where the commissural fibers are crossing over in the pars crassa of the lamina terminalis, there is no evidence of any other crossing fibers, either of the corpus callosum or of the hippocampal commissure (fig. 21 $F$ ). Dorsal to the anterior commissure are the anterior pillar of the fornix (af), the long interventricular canal of the foramen of Monro, $(i c)$, the cephalic portion of the third ventricle $(V B)$ and the anterior horn of the lateral ventricle. Forming the mesial wall of the lateral ventricle is the hippocampal formation. Its several areas, including the presubicular $(P S)$, the subicular $(S)$, the crescentic $(C)$, fasciolar $(F)$ and dentate $(D)$ areas, bear their usual relations to the hippocampal fissure $(H)$. Subjacent to the dentate area is the fimbria $(f)$ and, in its typical subependymal position, is the alveus, only partially developed as yet. The characteristic position of the interventricular canal as it forms the connection between the third and the lateral ventricles is shown in figure $21 \mathrm{E}$. A short distance in front of this canal (375 micra) the fibers of the hippocampal commissure ( $H C$ in fig. $21 D$ ) have established an ample crossing, the hippocampal formations of the two sides have drawn closer together and the hippocampal eminence is considerably reduced in size.

Still further toward the frontal pole of the hemisphere (500 micra) the caudal-most fibers of the corpus callosum make their appearance $(C C$ in fig. 21C). They form a small but well defined strand lying upon the dorsum of the hippocampal commissure $(H C)$ and supporting upon their own dorsal surface the dentate and fasciolar areas of the hippocampal formation ( $D$ and $F$ in fig. $21 C$ ). Thus it is obvious that, in the pig, the earliest developed fibers of the corpus callosum appear in the anterior quadrant of the mesial surface of the end-brain in front of the interventricular canal (foramen of Monro) and the anterior commissure. These crossing fibers interpolate themselves between the hippocampal commissure and the ventral portion of the hippocampus.

The manner in which the genu of the corpus callosum takes form is illustrated in figure $21 B$. Here the upper and lower divisions of the callosal fibers approach each other, fuse and cross the midline, $(C C$ in 


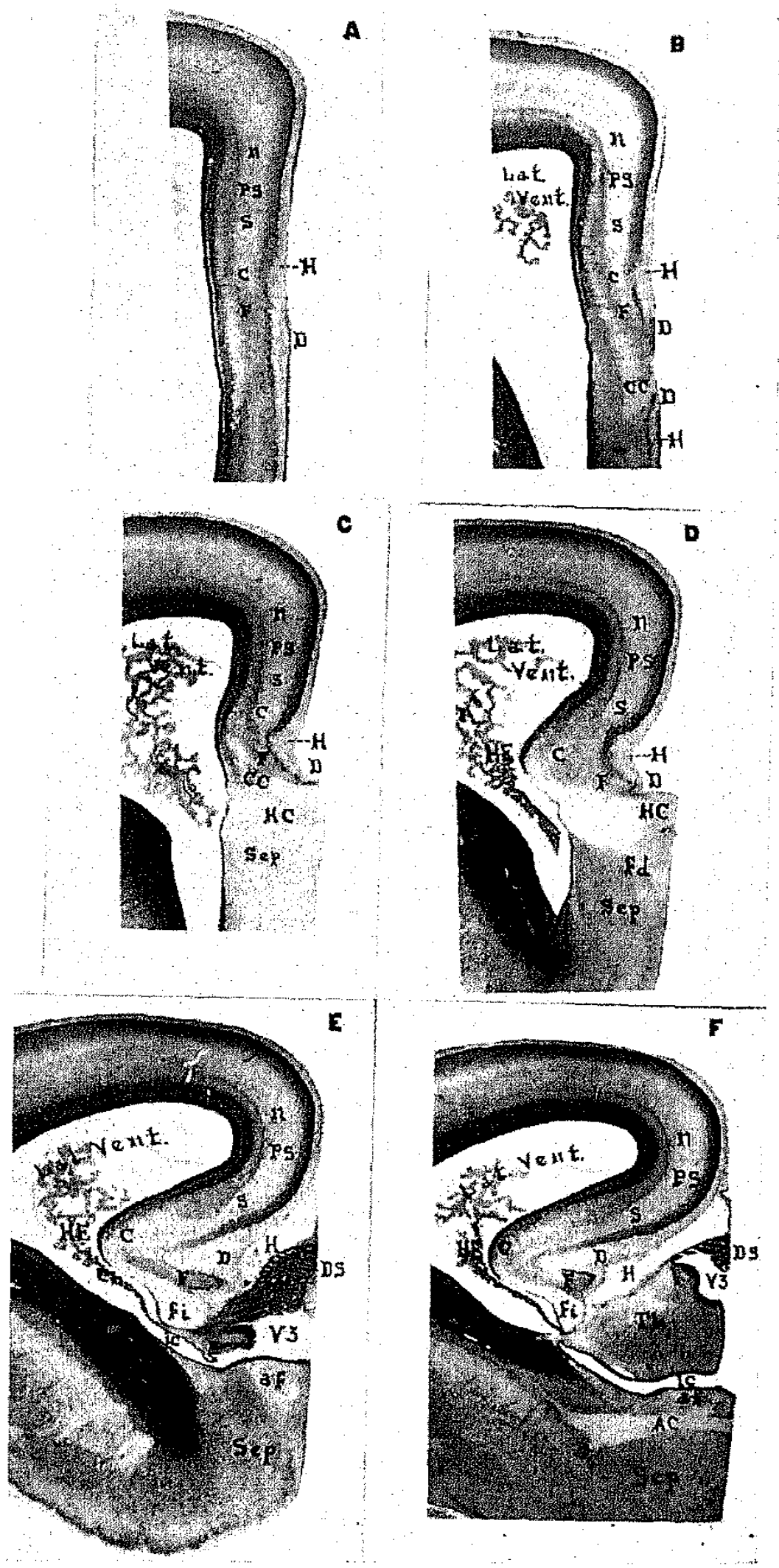

Fig. 21. Transverse Sections of 83 mim. Pig Embryo Show ing Early Appearance of Corpus Callosum

Specimen No. 2079, sections $140,150,180,195,210$ and 220 . Magnification $\times 15$. $A C$, anterior commissure; af, anterior fimbria; $c$, orescentic area; $c c$, corpus callosum; $c h$, chorioid plexus; $D$, dentate area; $D S$, dorsal sac; $F$, fasciolar area; $f d$, fimbrio dentate fissure; $f$, fimbria; $H$, hippocampal fissure; $H C$, hippocampal commissure; $H E$, hippocampal eminence; $i c$, interventricular canal; $n$, neocortex; $P S$, presubicular area; $S$, subicular area; $S e p$, septum; $T h$, thalamus; $V B$, third ventricle. 
fig. $21 B$ ). In making this crossing the callosal fibers pass through the hippocampal formation in such a way as to divide it into a supracallosal and a subrostral portion. Immediately in front of the genu these two portions of the hippocampal formation are reunited and assume the characteristics of the archicortex before it has felt the effects of introversion caused by the deepening of the hippocampal fissure. ( $D$ in fig. 21A.)

In following the further development of the hippocampus and its changes incident to the growth of the corpus callosum, certain principles should be borme in mind; first, that the crossing callosal fibers begin to appear in and, for a considerable period, are exclusively confined to the anterior quadrant, i.e., the portion of the mesial wall in front of the foramen of Monro and the anterior commissure; second, that these crossing fibers penetrate the mesial wall of each hemisphere in such a manner as to pass immediately beneath the hippocampus with the consequence that the archicortex and its adnexal structures are situated dorsal to the corpus callosum; and third, that the bridge provided for the actual cross ing of the callosal fibers appears to be made up of cells in the epithelial area which spans the gap between the two hemispheres.

As growth proceeds the archicortex covering the hippocampus is subject to several decisive influences which modify its general configuration without, however, in any way changing its essential relations or characters.

1. The caudal extension of the corpus callosum as it grows backward into the superior quadrant, i.e., behind the foramen of Monro, carries with it the growing structures of the archicortex located upon its dorsal surface. 2. The growth of the optic thalamus, from the first appearance of the dorsal sac to the expanded caudal end of the pulvinar, causes a divergence in the hippocampus originally occupying the superior quadrant. This original portion of the hippocampus which occupies the superior quadrant maintains its connection with the portion of the hippocampus situated above the corpus callosum. 3. The general growth of the brain wall causes both the hippocampus and the corpus callosum to be flexed or bent in such a way as to produce certain definite flexures in these structures. The flexures in the corpus callosum consist of the genu, the rostrum, the trumcus and the splenium. The flexures of the hippocampus, which in the main are best named from their relations to the corpus callosum, include the subrostral, the precallosal or pregenual, the supracallosal, the postcallosal or post-splenial, the subcallosal, the subventricular, the trigonal and the temporal flexures.

Neither the hippocampus nor the corpus callosum is changed in any of its fundamental features as it appears in these several flexures. In this sense a unitary character dominates both structures. There can be no doubt but that structurally the corpus callosum is identical throughout its entire extent. The only differences in the hippocampus are dependent 
upon the degree to which the archicortex, in its several flexures has had opportunity to develop.

\section{PROGRISSIVE CHANGES IN THE HIPPOCAMPUS OF THE IEMBRYONIC PIG BRAIN UP TO THE ADULT STAGE}

Each step in the advancing growth of the hippocampus may be followed through several embryonic stages up to adult conditions. Certain comparative measurements of structures in the growing pig's brain furnish precision in estimating the extent of the developmental process.

The absence of any crossing callosal fibers up to the $70 \mathrm{~mm}$. pig embryo stage indicates the protracted length of time during which the pig brain remains in its precallosal phase while the hippocampus retains its strictly marsupial character. The corpus callosum has, however, made its appearance in the $83 \mathrm{~mm}$. stage in which all of the crossing callosal fibers are limited to the anterior quadrant.

\section{The corpus callosum in the $90 \mathrm{~mm}$. pig embryo}

The $90 \mathrm{~mm}$. stage is not materially different from the $83 \mathrm{~mm}$. stage except for such advances as occur due to the increases of growth. The corpus callosum is still exclusively confined to the anterior quadrant and therefore occupies a position in front of the foramen of Monro and the anterior commissure.

All of the eight hippocampal flexures are distinguishable and the corpus ${ }^{1}$ callosum bears its characteristic relation to the hippocampus. Above it and resting on its dorsal surface is the supracallosal flexure; in front of it, the precallosal or pregenual flexure, while below the rostrum is the subrostral flexure. The structural features of the archicortex are evident in each of these flexures and the several areas typical of archicortical development may be easily identified.

One fact about the portions of the hippocampus in relation with the corpus callosum is to be noted, namely that while the characteristic fea- . tures of the archicortex may be readily discerned, they are less well developed than in the hippocampal regions which as yet have no contact or direct relation with the corpus callosum. Thus the hippocampal areas in the superior, posterior and inferior quadrants, all of them caudal to the foramen of Monro, have greater volume and structural distinctness. The introduction of the corpus callosum apparently retards the growth of the hippocampus but it does not otherwise alter its differentiation, so that the archicortex in contact with the corpus appears like hippocampal formation in miniature as contrasted with archicortex lacking such contact.

Another important factor in the development of the hippocampus is the divergence which occurs in the mesial walls of the hemispheres as the region of the thalamus is reached. This divergence begins with the ap- 
pearance of the cephalic extremity of the dorsal sac and continues to increase rapidly through the entire length of the dorsal thalamic surface. The effect is striking and critical. The hippocampal formations, which, in the anterior quadrant, have had a parallel course in close apposition to each other, now separate and continue to extend backward through the superior and posterior quadrants, diverging at an angle of approximately 45 degrees.

In the $90 \mathrm{~mm}$. pig embryo the growing corpus callosum has not yet entered the superior quadrant nor has it reached the caudal extremity of the hippocampal commissure. Nevertheless the continuity between the hippocampus which rests upon the corpus callosum is still maintained with the portions undergoing divergence. This continuity is established by a portion of the hippocampus which later becomes the postcallosal or postsplenial flexure. Inasmuch as the corpus callosum is still too inmature in its development to permit recognition of the splenium, it appears more conservative to speak of this connecting portion of the hippocampal formation as the postcallosal flexure.

\section{The corpus callosum in the $125 \mathrm{~mm}$. pig embryo}

Accepting the foramen of Monro as the point marking the boundary between the anterior and superior quadrants of the mesial surface, the corpus callosum, in its entirety, is confined to the anterior quadrant in the $125 \mathrm{~mm}$. pig embryo.

Heretofore, in earlier stages, (83 and $90 \mathrm{~mm}$. embryos) the caudal extremity of the corpus callosum has not extended so far back as the caudal extremity of the hippocampal commissure; now, however, the two great commissures are coextensive, i.e., their caudal extremities extend the same distance behind the foramen of Monro. This relation is maintained through all of the stages of further growth, with the result that the splenium of the corpus callosum consists of two distinct groups of fibers, superimposed one above the other; the callosal fibers occupy the dorsal position and the fibers of the hippocampal commissure, the ventral position.

All of the flexures of the hippocampus, due to the introduction of the corpus callosum, are well defined. The postcallosal and subcallosal flexures have acquired greater distinctness because of the caudal extension of the corpus callosum. At the same time the increasing divergence of the hippocampal formation in the superior quadrant, i.e., behind the foramen of Monro, gives greater prominence to this portion of the archicortex.

The hippocampal eminence first appears well forward in the anterior horn of the lateral ventricle. It makes a gentle projection into the ventricle, separated from the corpus callosum, which lies above it, by the 
hippocampo-callosal sulcus. As it extends backward the eminence increases in size until it forms a long tongue-like process projecting into the ventricle. This process, in its most cephalic portion, consists exclusively of nerve fibers but eventually forms an intimate association with the archicortex of the subventricular flexure and, maintaining this close association, passes downward into the trigonal portion of the lateral ventricle.

The several archicortical constituents appear in all eight of the hippocampal flexures. In all of these flexures it is possible to identify the alveus and fimbria as well as the presubicular, subicular, crescentic, fasciolar and dentate areas. In the superior, posterior and inferior quadrants these areas attain their most prominent development, while in the anterior, where they are in close contact with the growing corpus callosum, they give the impression of definite retardation in growth. Such retardation affects especially the subrostral, precallosal, supracallosal, postcallosal and subcallosal flexures. But even in these parts of the archicortex, growth continues and keeps pace with the growing corpus callosum until it reaches the limits of adult development. It is incorrect, therefore, to look upon the callosal portions of the hippocampus, in any true sense as atrophic. Rather is it the case that, because of the retarding influences of the corpus callosum, they have had less than their full opportunity to develop as they do in non-callosal mammals, like the opossum.

The hippocampal fissure, in all stages of development, serves as a reliable landmark. It occupies its usual position and bears its characteristic relation to the presubicular and dentate areas in all quadrants. In the superior, posterior and inferior quadrants, the fimbrio-clentate fissure is well defined.

\section{The corpus callosum in the $140 \mathrm{~mm}$. pig embryo}

In the pig's brain of this stage, the first really critical change in the growth of the corpus callosum takes place. The caudal extremity of the callosum which, prior to this time, has occupied a position in the anterior quadrant, in front of the foramen of Monro, has now extended backward into the superior quadrant. By measurement it lies 2750 micra behind the foramen whereas in the $125 \mathrm{~mm}$. embryo it is situated 625 micra in front of this foramen. In consequence of this change the postcallosal and subcallosal flexures have become more conspicuous (fig. 22, Specimen 2071, sections 465 and 470 ). Whether it is justifiable to call the caudal extremity of the corpus callosum, before it has attained its full growth, the splenium, is somewhat questionable. Doubtless, there are still more crossing callosal fibers to enter the structure from the parietal and occipital regions of the hemispheres. For this reason, the growth of the corpus callosum caudad appears to be something more than a mere pari passu expansion with the fronto-caudal growth of the brain. However, this 

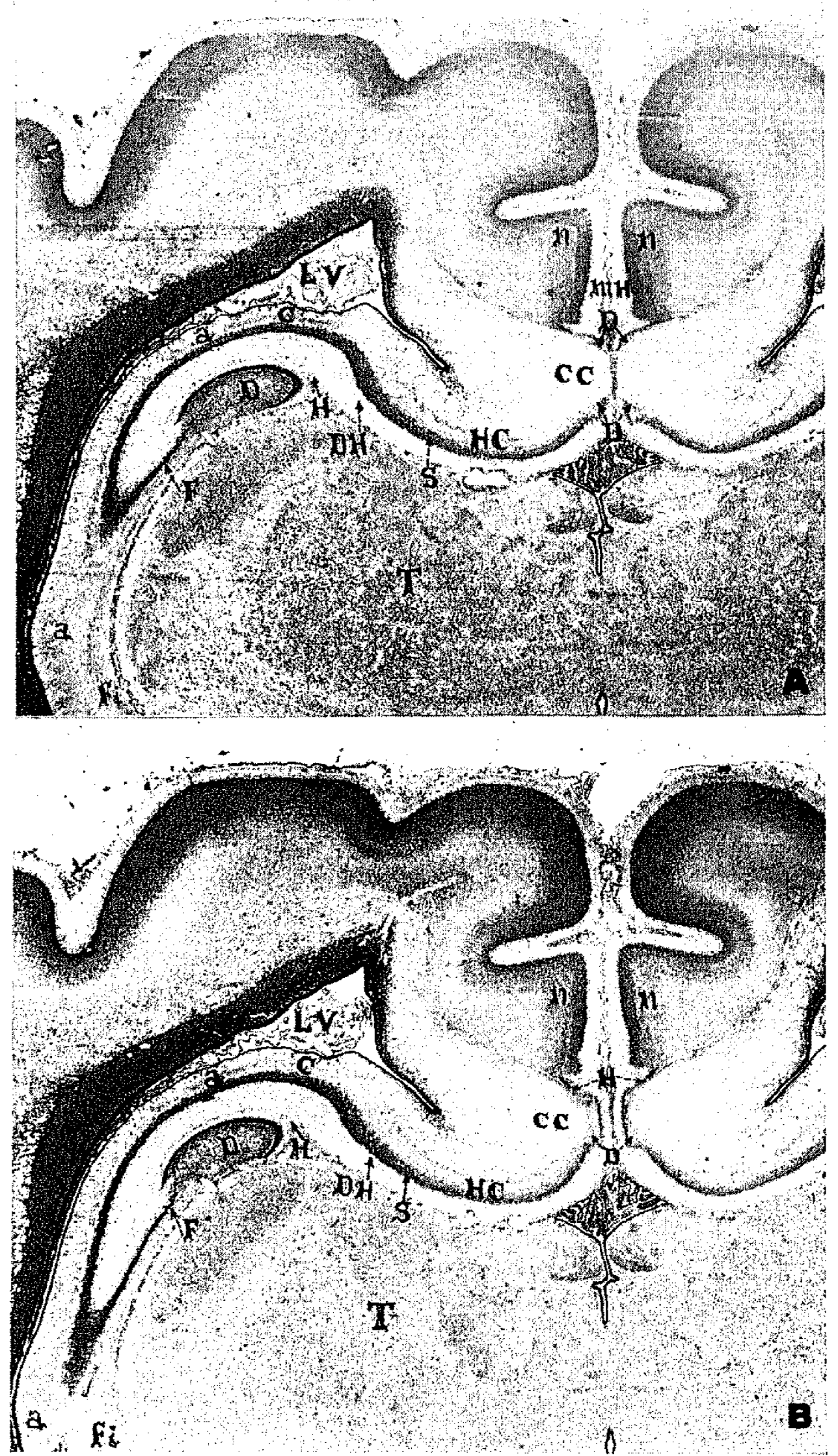

Fig. 22. Transverse Sections Showing Midline and Divergent Hippocampat. Divisions in 140 Mm. Pig Embryo

Specimen No. 2071, sections 465 and 470 . Magnification $\times 12$. a, alveus; $C$, crescentic area; $c c$, corpus callosum; $D$, dentate area; $D H$, divergent hippocampal

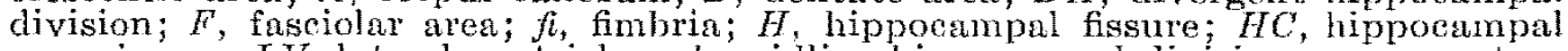
commissure; $L V$, lateral yentricle; $m h$, midline hippoeampal division; $n$, neocortex; $S$ subicular area; $T$, thalamus. 
may be, the caudal extremity of the callosum has most of the characteristics of the splenium, namely, 1, a dorsal group of callosal fibers overlying a ventral group which enters into the hippocampal commissure; 2 , a post-callosal flexure of the hippocampus caudal to the callosum which connects a supracallosal with a subcallosal flexure; and 3, an approximation of these several flexures close to the midline. If, as is presumably the case, additional fibers are still to enter the corpus callosum from the parietal and occipital lobes, they may span the gap between the hemispheres, either by utilizing the callosal fibers already present or by penetrating the bridge of cells in the epithelial area which is situated dorsal to the corpus. These, however, are not necessarily problems of the first order', and the solutions here suggested for them may be sufficient for the present.

This stage is significant, therefore, because it marks the extension of the corpus callosum into the superior quadrant with the expansion of the supracallosal, the postcallosal and the subcallosal flexures. The further divergence of the subventricular flexure makes clear the reasons for its fuller developmental appearance. It also calls attention to the opportunities afforded for its further growth in contrast to those portions of the archicortex which maintain close relations with the corpus callosum and remain close to the midline. Equally important is the continuity maintained between the callosal portions of the archicortex and the subventricular flexure. Structurally this continuity results in the formation of a long loop which begins with the postcallosal flexure. It then swings forward bencath the corpus callosum as the subcallosal flexure and turns sharply outward to join the subventricular flexure which extends backward beneath the body of the lateral ventricle until it arches downward into the trigonum ventriculi.

\section{The corpus callosum of the $165 \mathrm{~mm}$. and $185 \mathrm{~mm}$. pig fetuses}

In the fetal pig brain growth has determined proportional expansions in all of the hippocampal flexures and, at the same time, produced greater cellular differentiation in all archicortical areas. The distinctions already recognized, now become so striking as to put the unitary character of the cortex covering the hippocampus entirely beyond dispute. From one end of the archicortex to the other, from the subrostral, through the precallosal, supracallosal, postcallosal, subventricular, trigonal and temporal flexures, the same succession of areas characterizes the tissue. The typical relations of these areas to the hippocampal and fimbrio-dentate fissures are maintained, while the intraventricular sulci hold their identifying positions relative to the several features of the ventricle.

In the $165 \mathrm{~mm}$. pig fetus the caudal extremity of the corpus callosum has extended 3500 micra behind the foramen of Monro, while in the 
$185 \mathrm{~mm}$. pig fetus the post-foraminal distance is 4500 micra. Both of these stages show, with greatest clearness, the manner in which the supracallosal flexure is continued around the caudal end of the corpus callosum and then forms the long subcallosal loop connecting with the subventricular flexure (see table 1).

TABLE 1

Cephalo-caudal growth of structures in the hippocampal region in the pig

\begin{tabular}{|c|c|c|c|c|c|c|c|}
\hline & \multicolumn{7}{|c|}{ TOTAL LENGTH OF ANIMAL } \\
\hline & $\begin{array}{c}83 \\
\mathrm{~mm} .\end{array}$ & $\begin{array}{c}90 \\
\mathrm{~mm}\end{array}$ & $\begin{array}{c}125 \\
\mathrm{~mm}\end{array}$ & $\begin{array}{c}140 \\
\mathrm{~mm} \text {. }\end{array}$ & $\begin{array}{c}185 \\
\mathrm{~mm} .\end{array}$ & $\begin{array}{c}225 \\
\text { mm. } \\
\text { (new } \\
\text { born) }\end{array}$ & Adult \\
\hline & micra & micra & miera & micra & micra & micra & micra \\
\hline Corpus callosum.... & 750 & 1,360 & 1,425 & 6,125 & 8,375 & 13,750 & 25,800 \\
\hline Supracallosal flexure. & 625 & 1,340 & 1,420 & 6,115 & 8,360 & 13,740 & 25,500 \\
\hline Subrostral flexure.... & 375 & 440 & 625 & 1,000 & 2,125 & 2,250 & 3,450 \\
\hline Subcallosal flexure........ & $*$ & $*$ & 50 & 1,000 & 2,000 & 3,125 & 6,750 \\
\hline Subventricular flexure.... . & 750 & 980 & 1,000 & 1,200 & 1,375 & 1,875 & 5,100 \\
\hline Hippocampal commissure...... & 775 & 860 & & 1,575 & 4,625 & 7,675 & 10,200 \\
\hline \multirow[t]{2}{*}{ Anterior commissure............. } & 250 & 280 & 300 & 375 & 375 & $500 \mid$ & 650 \\
\hline & \multicolumn{3}{|c|}{ IN DRONT OF FORAMEN } & \multicolumn{4}{|c|}{ BACK OF FORAMEN } \\
\hline $\begin{array}{l}\text { Relation of caudal extremity of cor- } \\
\text { pus callosum to foramen of Monro.. }\end{array}$ & 1,125 & 620 & 625 & 2,750 & 4,500 & 7,750 & 15,600 \\
\hline
\end{tabular}

* Not yet present.

\section{Corpus callosum in the newborn and adult pigs}

The brains of the newborn and adult pigs complete the story of callosal growth. With the exception of relative differences in size, the one duplicates the other. For example, in the adult the caudal extremity of the corpus callosum lies 15600 micra behind the foramen of Monro, while in the one day old suckling this distance is 7750 micra. A tabulation of the comparative lengths of the several essential structures in the hippocampal region from the $83 \mathrm{~mm}$. stage to full maturity is shown in table 1 . Through these figures it is possible to trace the gradients of hippocampal development as they affect the pig brain.

The process of growth shows clearly that the corpus callosum begins its development in the anterior quadrant and thus in a portion of the mesial surface of the hemisphere in front of the foramen of Monro and the anterior commissure. It also indicates that the crossing callosal fibers bridge the gap between the two hemispheres either by traversing fibers of the hippocampal commissure already laid down or by using cell bridges formed by the epithelial area. Before passing over from one hemis- 
phere to the other, the fibers destined to form the corpus callosum penetrate the mesial wall at a level immediately in front of the hippocampal formation and thus when they do cross, they, so to speak, carry the hippocampus on their back.

The fibers which enter and form the corpus callosum all arise in the neocortex. With reference to the localities of their origin, they spring from three main sources, namely, the neocortex above the septum, the neocortex in front of it and the neocortex below it. The callosal fibers derived from these several cortical regions form a lamina of considerable thickness which, according to its topographical relations, presents three rather distinct divisions, i.e., the dorsal division, the frontal division and the ventral division. All three of these divisions converge upon the centrum callosum, which consists of a collection of fibers about to make their way through the mesial wall to enter the corpus callosum. In the adult, the fibers forming the callosal lamina are situated immediately beneath the ependymal lining of the ventricle and constitute what is usually called the tapetum. Before entering the corpus callosum, the fibers from the neocortex above the septum pass downward into the centrum callosum; the fibers from the cortex in front of the septum pass backward while the fibers from the cortical areas below the septum extend upward. This arrangement of fibers entering the corpus callosum obtains in the anterior quadrant only. It results in the formation of the rostrum, genu and anterior portion of the truncus.

In the superior quadrant, the fibers entering the corpus callosum make their way to the centrum callosum from the parietal, temporal and occipital lobes. Their approach to the centrum from the occipital region takes the form of a heavy lamina resembling that in the frontal lobe and called the forceps major. The fibers from the occipital lobe, upon entering the caudal extremity of the corpus callosum, form a thickened portion. of that body which, with the addition of the caudal fibers of the hippocampal commissure, constitute the splenium. The portion of the corpus callosum between the splenium and the genu forms the truncus.

\section{Fiber systems of the hippocampus}

Throughout its entire extent the corpus callosum is in close contact with the fiber systems of the hippocampus. These fiber systems are two in number, namely, the alveal system forming the alveus and the fimbrial system forming the fimbria. Under cover of the ependymal lining, the alveus forms the floor of the ventricle just as the tapetum of callosal fibers forms its roof. This statement should necessarily be modified because of the several curves in the lateral ventricle.

In the anterior quadrant the hippocampal fiber systems occupy the dorsal portion of the septum and lie immediately beneath the corpus 
callosum. A shallow furrow in the lateral ventricle, the hippocampocallosal sulcus, partially separates the callosal and hippocampal fibers although, in larger part, they are in direct contact. The hippocampal fibers, in the superior quadrant, form a long tongue-like process extending into the lateral ventricle, and spring from a broad central base closely adherent to the under surface of the corpus callosum. The more dorsal fibers in the base and tongue-like extension of this process belong to the alveal system while the ventral fibers are constituents of the fimbrial system.

Both the subcallosal and the subventricular flexures of the hippocampus actually bear a contact relation with these hippocampal fiber systems. Furthermore the splenium of the corpus callosum does not consist exclusively of callosal fibers, but in its ventral portion is largely augmented by fibers constituting the hippocampal commissure. In the strict sense, however, the hippocampal fiber system of the pig is commissural from its first appearance in the septum to its termination in the splenium.

If a simile might prove at all helpful, it could be said that the callosal and hippocampal fiber systems resemble two parallel sets of wings, the upper or callosal set, spread out above the body of the lateral ventricle, the lower or hippocampal set, extending beneath the ventricle, but both sets joined at the middle to a long, stout body. The ventral part of this body surrounds the subcallosal flexures of the right and left sides, while each wing of the lower set embraces the respective subventricular flexure of the hippocampus.

\section{The hippocampal formation}

The analysis which seems to lend itself to most effective description, subdivides the hippocampal formation into two major divisions: 1, a midline hippocampal division which, because of the development of the corpus callosum, persistently holds its position close to the midline of the brain; and 2, a divergent hippocampal division which, under the influence of thalamic development, tends to diverge more or less from the midline.

Added to the effects of these factors must also be considered the results of flexures which occur in the corpus callosum and in the brain as a whole.

Such an analysis, based on the developmental stages of the pig's brain, demonstrates clearly how the relatively simple conditions of the noncallosal brain, such as that of the opossum, assume the far more complex dispositions of the mammalian brain in general.

Followed quadrant by quadrant, the hippocampal changes of the callosal brain stand out with distinctness. In the anterior quadrant, i.e., in front of the foramen of Monro, these changes confine themselves exclusively to the midline division of the hippocampus. They are determined by two factors, first, the crossing of the callosal fibers which first 
appear in this quadrant, and second, the flexions which occur in the corpus callosum due to growth of the mesial wall of the brain. The crossing callosal fibers pass beneath the hippocampal formation in such a manner as to surround all of the archipallial structures. All of the archicortical areas as well as the hippocampal fiber-systems, the alveus and the fimbria, thus lie inside of or above the encircling callosal fibers.

The transition from the neocortex to the archicortex has its typical characteristics and the hippocampal fissure serves as the usual landmark in relation to the several archicortical areas. There is no difficulty in distinguishing the presubicular, subicular, crescentic, fasciolar and dentate areas. Even the usual detachment between the fasciolar and dentate areas has occurred and the intercalary area is discernable in places.

Curvature in the corpus callosum determines three flexures in the hippocampus of this region, namely, the subrostral, the precallosal and the cephalic portion of the supracallosal flexure. The hippocampal fissure is less deep in the precallosal and subrostral areas with the consequence that the several archicortical regions occupy more exposed positions in the mesial wall of the hemisphere.

The corpus callosum, as it extends into the superior quadrant, affects both the midline and the divergent divisions of the hippocampus. Its progressive growth caudad, during later stages of development, is accompanied by corresponding growth in all of the hippocampal structures. The appended tabulation (table 1 ) indicates the comparative rates of expansion in certain outstanding structures of the hippocampal region. From these figures it is obvious that actual growth has occurred in all portions of the midline division of the hippocampal formation. This fact seems to have definite significance. It apparently justifies the view that this division of the hippocampus is not necessarily retrograde in function but since it has kept pace structurally with adjacent tissues, may still retain some of its inherent activities. As the figures show, there has occurred a great expansion in the supracallosal flexure while passing from earlier embryonic stages to adult life. Not only is this expansion marked, but, during its entire course of growth all of the salient histological features of the archipallium have retained their identity, have, indeed, gained both in the definition and maturity of their essential characteristics. Such persistency in tissue viability does not predicate the vestigal tendencies usually attributed to this portion of the brain which, in general usage, is somewhat disparagingly called the indusium griseum. A similar viability occurs in other portions of the midline hippocampal division. The postcallosal flexure expands with the expansion of the splenium and likewise maintains the integrity of all of its archicortical areas; then, as the subcallosal flexure, it swings forward beneath the splenium, still in close apposition to the midline. This loop-like part of the hippocampus, first pass- 
ing around the splenium and then extending forward beneath it, forms the connecting link with the divergent hippocampal division.

In the light of developmental events, it is beyond doubt the successive increments of callosal fibers which supply the main increases in the cephalo-caudal length of the corpus callosum. This factor also underlies the progressive additions in all structures belonging to the midline division of the hippocampus.

The loop established by these portions of the hippocampal formation, including the supracallosal, postcallosal and subcallosal flexures, lies in the vertical plane. Another and, in many respects, still more conspicuous hippocampal loop occupies this same general region beneath the corpus callosum. In this case, however, the plane of the loop is horizontal and extends outward below the body of the lateral ventricle. The relations of these two loops have led to much confusion in description and to unfortunate superfluity in nomenclature. The inner limb of the horizontal loop which is formed by the subcallosal flexure of the hippocampus extends forward, in a midline position, for a considerable distance beneath the corpus callosum. Ultimately it is joined by the diverging portion of the hippocampal formation which at first turns outward at right angles under the lateral ventricle and then sweeps backward as the subventricular flexure. The flexures in the caudal region of the corpus callosum thus form two highly important loops which participate in the transition from the midline to the diverging portion of the hippocampal formation.

These two loops may be traced in their continuity as follows: Beginning with the supracallosal flexure as the superior limb of the vertical loop, the postcallosal flexure arches around the splenium and, still in a midline position, is carried forward as the subcallosal flexure beneath the corpus callosum. This flexure is also the inner $\operatorname{limb}$ of the horizontal loop and thus the connecting link between the midline and diverging portions of the hippocampus.

The distinction between the vertical and horizontal loops is striking. It does not, however, depend on the differences in the kind of cellular specialization, for in both regions all of the archicortical areas can be identified. In the horizontal loop the hippocampal tissues have escaped the retarding effects imposed by contact with the corpus callosum and have been afforded more ample opportunity to expand by their extension into the lateral ventricle where they form the prominent hippocampal eminence. All of the histological characteristics of the hippocampus are more highly developed in the horizontal than in the vertical loop and the several structures have attained greater dimensions.

As the hippocampal formation approaches the trigonum of the lateral ventricle, it makes another sharp bend, this time downward, to form the trigonal flexure. Here the hippocampus lies behind the optic thalamus. 
It retains all of the highly specialized features of the subventricular flexure and projects into the ventricle to form the most conspicuous portion of the hippocampal eminence.

On reaching the temporal region the hippocampus undergoes its last major flexion. It swings forward in relation to the temporal horn of the lateral ventricle and extends forward to its tip. This part of the hippocampal formation constitutes the temporal flexure.

It has been shown that, in the pig's brain, the hippocampus, which represents the archicortex, retains its structural integrity throughout its entire extent.

This structural integrity is based upon the conditions of the noncallosal brain, such as that of the opossum. It includes the differentiation of six specific archicortical areas, i.e., 1 , the presubicular; 2 , the subicular; 3 , the crescentic; 4 , the fasciolar; 5 , the intercalary and 6 , the dentate areas. To these six archicortical areas should be added the specialization of two hippocampal fiber systems, namely, the alveal system and the fimbrial system.

The hippocampus in the pig's brain passes through a precallosal phase of considerable duration, in which the disposition of the archicortex closely resembles that of the marsupial. In particular, it manifests flexures in the frontal, parietal, occipital and temporal regions of the mesial surface of the hemisphere.

The introduction of the corpus callosum increases the number of these flexures and otherwise complicates their relations.

The flexures directly influenced by the development of the corpus callosum are five in number, namely, 1 , the subrostral; 2 , the precallosal; 3 , the supracallosal; 4 , the postcallosal; and 5, the subcallosal flexure. All of these regions of the hippocampus in consequence of their intimate relation with the corpus callosum retain a close connection with the midline of the brain. It is advantageous for several reasons to distinguish them collectively as the midline division of the hippocampus. This midline hippocampal division is in contrast with the divergent hippocampal division, because the latter diverges from the midline to such an extent that it forms a large protrusion into the body, trigonum and temporal horn of the lateral ventricle to form the hippocampal eminence. Furthermore the divergent division, because of its relations with the lateral ventricle, has opportunity to attain its full structural expansion, whereas the midline division is definitely retarded in its growth by reason of its relations with the corpus callosum.

The flexures of the hippocampus not influenced by the development of the corpus callosum are three in number, namely, the subventricular, the trigonal and the temporal flexures.

There are thus a total of eight flexures affecting the hippocampus. The 
objection to calling any or all of these flexures either distinctive gyri or convolutions with special names is revealed by the fact that they are, in the strict sense, parts of one and the same archicortical tissue with complete identicality of structure. Their only differences are incidental to their relations to the corpus callosum and lateral ventricle.

Every hippocampal flexure, whether in the midline or the divergent division, consists of the same series of archicortical areas in the following order from the outer to the inner margin of the hippocampus: 1 , the presubicular; 2 , the subicular; 3 , the crescentic; 4 , the fasciolar; 5 , the intercalary; and 6 , the dentate. The fimbrial and the alveal fiber-systems likewise enter into the formation of every flexure. The fimbria forms the actual inner margin of the hippocampus. In the anterior quadrant it is the most mesial hippocampal component and rests on the dorsal surface of the corpus callosum. It should be born in mind, however, that in the region of the genu and the rostrum this relation is changed to the anterior and ventral surfaces respectively. In this position the fimbria represents the structure usually designated the striae Lancisii.

In the subventricular, trigonal and temporal flexures, the fimbria has greater dimensions and lies in close relation with the dorsal surface of the thalamus from which it is separated by the chorioidal fissure. Part of the chorioidal plexus for the lateral ventricle passes through this fissure. The anterior portion of the fimbria extends forward above the roof of the third ventricle, sweeps around the foramen of Monro and, in greater part, behind the anterior commissure to enter the basal region of the interbrain. This portion of the fimbria is usually called the "anterior pillar of the fornix." In most mammalian brains below the higher primates, no such pillar, in fact no structure resembling the fornix or vault, can be distinguished, inasmuch as the fimbrial fibers run a much shorter course than in man and are more closely invested by adjacent structures. In the brain of the higher anthropoids and man, tie anterior pillar of the fornix is demonstrable, although its functional significance as part of the fimbrial system is definitely obscured by labeling it with a term which has justifiable application to a small group of mammals only.

The fibers of the alveal system are present in all flexures of the hippocampus. In the midline division, they lie internal to the descending callosal lamina. In the divergent division, they form a broad but thin sheet of nerve fibers covering the ventricular surface of the hippocampal eminence. As this sheet approaches the midline, it undergoes crossing and establishes the hippocampal commissure which extends forward, attached to the under surface of the corpus callosum, from the splenium to the septum.

The hippocampal fissure affords the most reliable landmark in the entire hippocampus. Its invariable relations to the several archicortical 
areas furnishes a consistent guide for the identification of the different components in this part of the brain.

VII. EVIDENCE FROM THE DEVELOPMENTAL HISTOIRY OF OTHER MAMMALS POSSESSING A CORPUS CALLOSUM

Equally strong evidence concerning the relations of the hippocampus to the corpus callosum is offered by other eutherian mammals. The developmental process of the brain has been similarly studied by the reconstruction method in the rat, guinea pig, cat and man.

In all of these mammals, which possess a corpus callosum, the brain passes through a relatively long precallosal phase, followed by a callosal stage "duplicating, in all particulars, the characteristics of development in the pig's brain.

Likewise, in all of these animals, the corpus callosum first appears in the anterior quadrant, i.e., in front of the foramen of Monro and anterior commissure. From this starting point it extends backward through the superior quadrant, i.e., behind the foramen of Monro. Its growth and expansion are attended by certain local specializations, such as the rostrum, the genu, the truncus and the splenium. In relation to these specializations, the hippocampus is essentially similar in all species. No essential differences occur in the development of the midline hippocampal division in any of these species.

For the most part, the divergent hippocampal division is marked by a striking similarity of structural detail in all mammals. In the human' brain, the subcallosal and subventricular flexures, such conspicuous features in other animals, are either absent or only slightly developed. Here also the postcallosal flexure crosses the splenium obliquely and thus joins the trigonal flexure directly. With these exceptions, the process of development in the corpus callosum and hippocampus in all eutherian mammals which I have examined is identical.

Reconstruction of the corpus callosum in adult mammatian brains especially prepared by the Pal-Weigert method

The adult brains of sixteen species, representing four different orders, were examined in serial sections prepared by the Pal-Weigert method. This procedure offered opportunity to study, with greater exactness, the nerve fibers entering into the hippocampus and, at the same time, to follow the structural details of this region of the brain in a fairly diversified group of mammals. Reconstructions by the wax-plate method were made of eight of these brains, the reproductions of which are given in figures 23 to 31 .

Tapir bairdi is a representative of one of the three living genera of perisodactyles, namely the horses, tapirs and rhinoceroses. These animals 
constitute the suborder of ungulates, characterized by the fact that the middle digit of the fore and hind foot is pre-eminent. It is for this reason that they are called odd-toed ungulates. The tapir belongs to a family fully as ancient as the horses and is, in many respects, the most ancient perisodactyle form at present inhabiting the earth. It lives in South and Central America, in the Malay Peninsula and in the islands of Java and Sumatra.

The hippocampus and corpus callosum in the tapir have all of the characteristic features of this cerebral region in the eutherian mammal (fig. 23A). The hippocampal formation, consisting of the typical succession of six archicortical areas, presents eight characteristic flexures. Striking features involving these flexures occur in certain regions. For example, the subrostral flexure occupies a more exposed position in the mesial wall than in mammals generally, although this disposition is not uncommon in many other animals because the hippocampal fissure is usually most shallow in this region of the brain.

The precallosal and supracallosal flexures are typical in all respects and especially characterized by a deep hippocampal fissure with its usual archicortical boundaries. In the remainder of the midline hippocampal division, including the postcallosal and subcallosal flexures, the structural modifications in the hippocampus are noteworthy. The most conspicuous change is due to the fact that the hippocampal fissure becomes extremely shallow in the postcallosal area and thus creates the impression that the fissure actually terminates in this region (fig. 23A). Such a condition is found in many mammalian brains. Indeed, in the same brain it may even be the case that the hippocampal fissure in this area of one hemisphere is well defined, but in the other hemisphere so shallow as to be a most indistinct landmark. This apparent failure of the hippocampal fissure to develop in the postcallosal area has led to certain misconceptions of this region. It has been asserted that the hippocampal fissure, not only terminates at this point but that the portion of it which appears above the corpus callosum is a neomorph. Nothing, in my opinion, could be further from the truth, for the apparent interruption of the hippocampal fissure is due to the fact that the fissure becomes so shallow that the crescentic area of archicortex comes to the surface of the mesial wall.

In general, it may be said that no actual interruption in the hippocampal fissure occurs in this posteallosal or any other region of the brain. The unbroken continuity of the fissure, from one end of the hippocampus to the other, may be established by careful histological studies of the archicortical areas forming the fissural boundaries.

The subcallosal flexure of the hippocampus, (fig. 23A) not only attains unusual dimensions in the dentate area but the subicular area is especially conspicuous ( $D$ and $S$ in fig. $23 A$ ). 


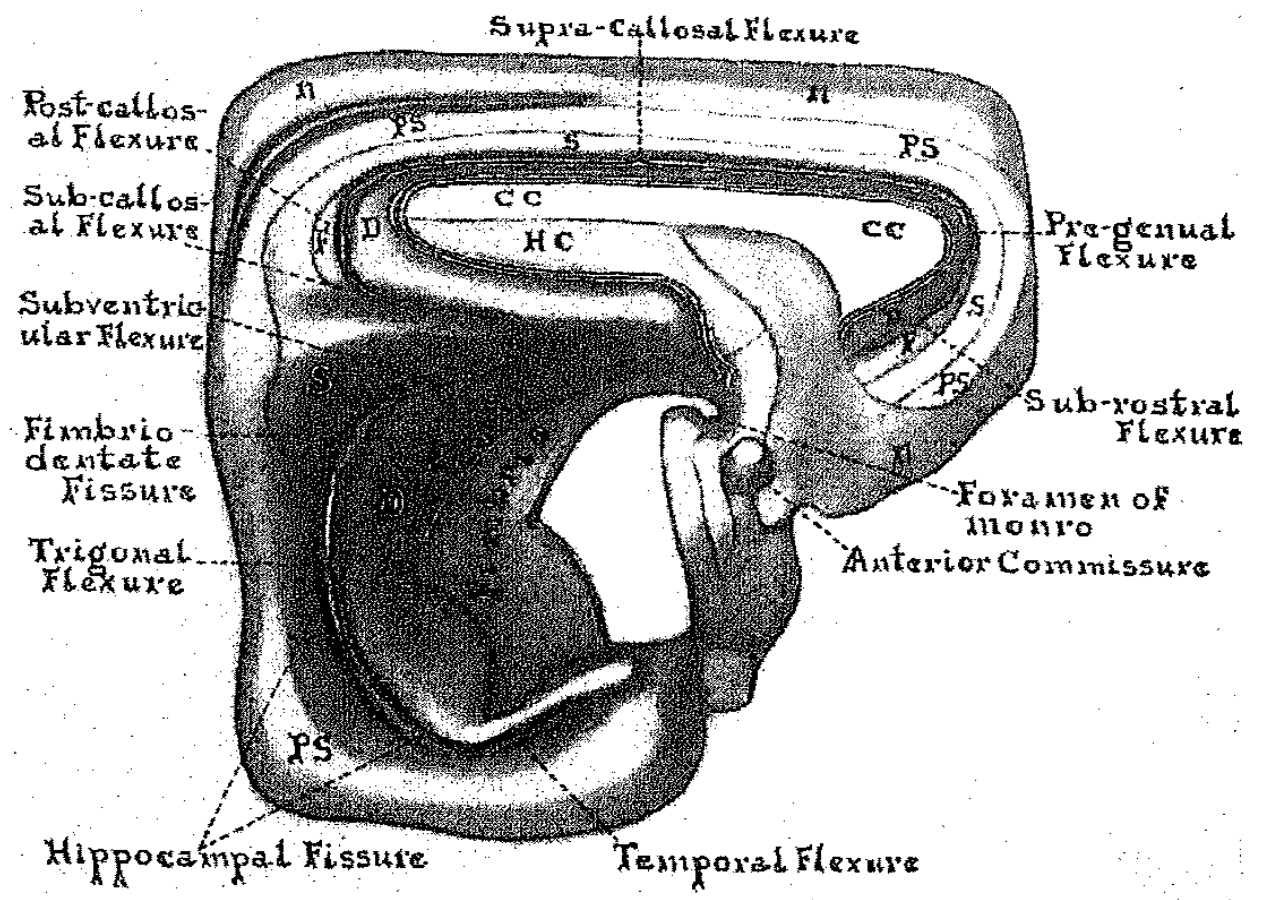

A

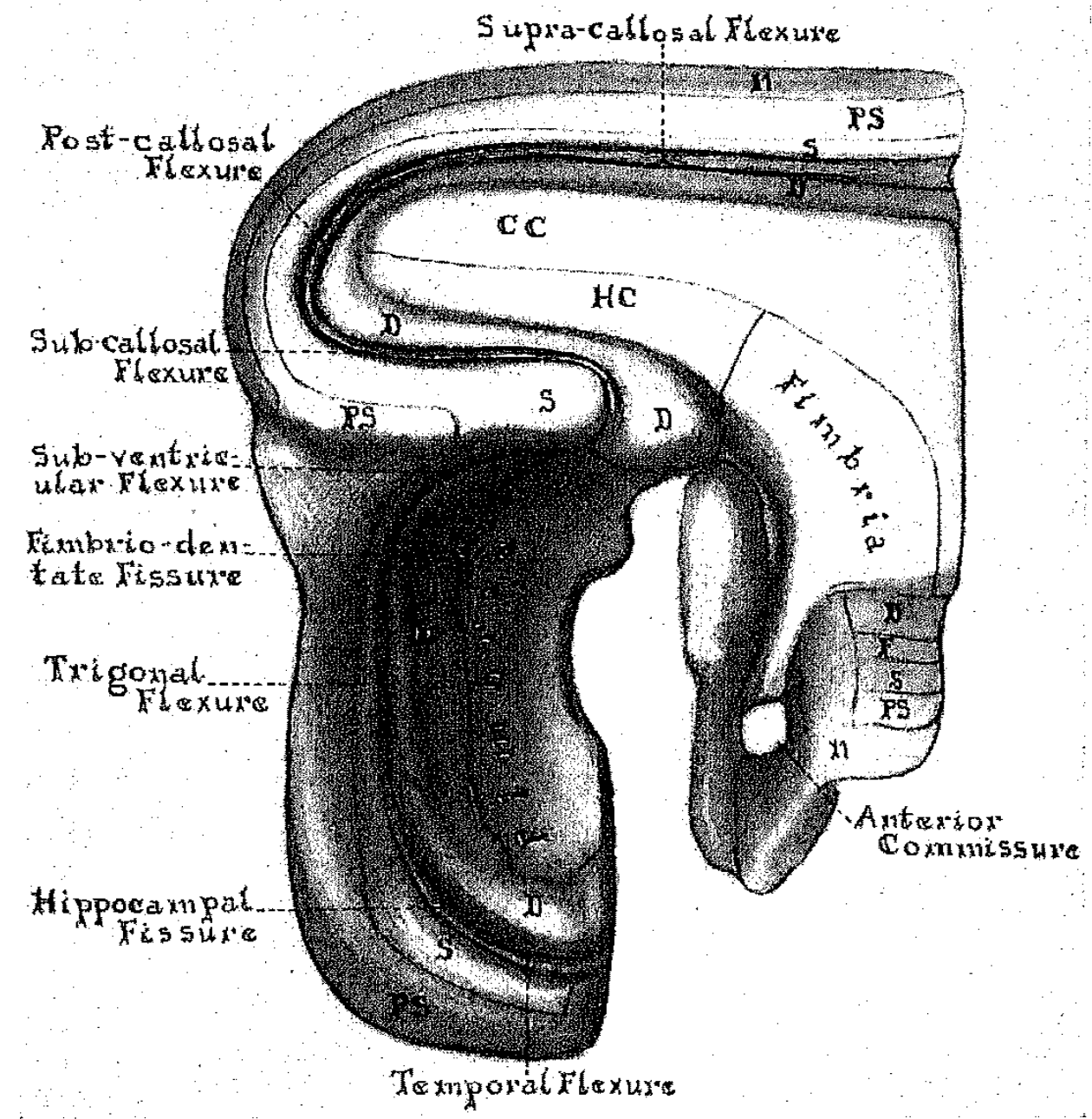

B

Fia. 23A. Reconstruction of Hippocampal Region in Adult Tapir Bairdi, Left HEMISPHERE

Specimen No. 247. Magnification $\times 5 . c c$, corpus callosum; $D$, dentate area; $F$, fasciolar area; $H C$, hippocampal commissure; $n$, neocortex; $P S$, presubicular area; $S$, subicular area.

Fig. 23B. Reconstruction of Hippocampal Region in Adult Horse, Left HEMISP HERE

Specimen No. 248. Magnification $\times 5 . C C$, corpus callosum; $D$, dentate area; $F$, fasciolar area; $H C$, hippocampal commissure; $n$, neocortex; $P S$, presubicular area; $S$, subicular area. 
In the divergent hippocampal division, the subventricular flexure effects its typical connection with the subcallosal flexure and forms the loop which eventually turns backward beneath the body of the lateral ventricle to descend into the trigonum ventriculi. At the lower extremity of the trigonal flexure, the hippocampus bends sharply forward to form the temporal flexure.

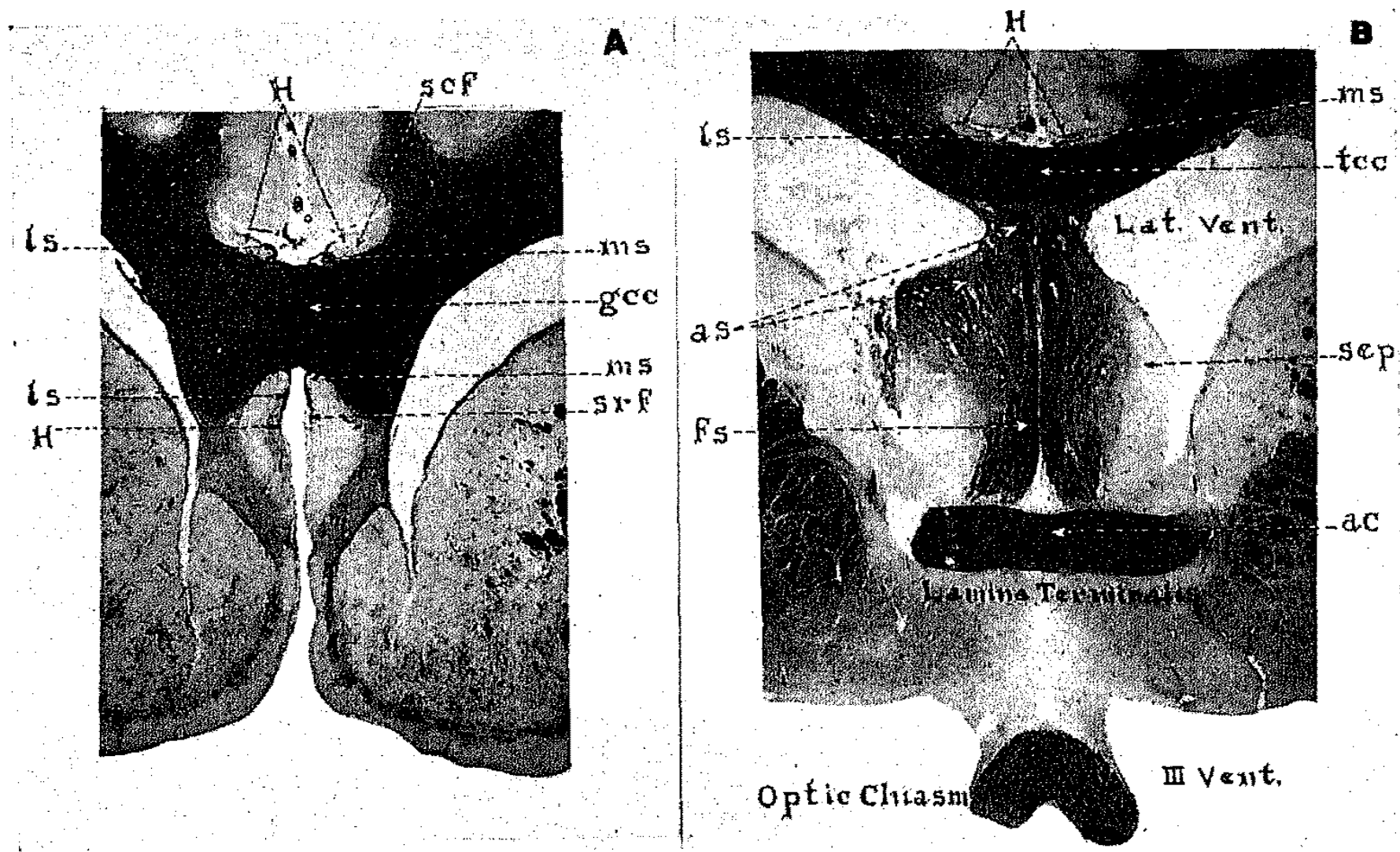

Fig. 24A. Cross Section Through Genual Region of Corpus Callosum in Tapir BAIRDI

Specimen No. 247. Slide No. 1185. Magnification $\times 4$. gcc, genu of corpus callosum; $H$, hippocampal fissure; $l s$, lateral stria; $m s$, medial stria; $s c f$, supracallosal flexure; srf, subrostral flexure.

Fig. 24B. Cross Section Through Anterior Commissure of Tapir Bairdi

Specimen No. 247. Slide 1085. Magnification $\times 4 \frac{1}{2}$. ac, anterior commissure; $a s$, alveal system; $f s$, fibrous septum; $H$, hippocampal fissure; $l s$, lateral stria; $m s$, medial stria; sep, septum; tec, truncus of corpus callosum.

The two hippocampal fiber-systems, forming the alveus and the fimbria respectively, occupy their characteristic positions and maintain their usual relations.

In the midline division of the hippocampus a slender cord of nerve fibers extends along the inner margin of the archicortex of the subrostral precallosal, supracallosal, postcallosal and subcallosal flexures. In its course, it lies mesial to the dentate area and in surface relief appears as a long, cord-like elevation usually referred to as the median stria or nervus Lancisii. Lateral to and running parallel with this fiber-bundle is a second 
and lesser elevation of nerve-fibers forming the lateral stria. Both of these long, narrow bundles participate in the formation of the fimbrial system. They are shown in cross-section in figure $24 A$ and $B$, ( $m s$ and $l s)$. The median and lateral striae are related to the midline division of the hippocampus. They may be traced from the beginning of the subrostral flexure, through the precallosal, supracallosal, postcallosal and subcallosal flexures. At the cephalic extremity of the subcallosal flexure they appear to enter the septal portion of the fimbrial system. In their further course, they pass behind the anterior commissure and are thence continued into the lateral wall of the third ventricle. The more detailed analysis of these striae must await further investigation of the fimbrial system as a whole.

In the divergent division of the hippocampus consisting of the subventricular, trigonal and temporal flexures, the fimbria as well as the archicortical areas are greatly increased in size.

The fimbrio-dentate fissure is well defined and the inner margin of the fimbria gives attachment to the chorioidal lamina which enters the trigonum and body of the lateral ventricle through the chorioidal fissure.

The alveal system of nerve fibers makes its first appearance in the lateral portion of the septum immediately in front of the foramen of Monro. It forms a fiber system apparently distinct from but intimately intermingled with the fimbrial system which occupies a mesial position in the septum. In the tapir the alveus retains its intraventricular position and forms a long sheet of nerve fibers which covers the hippocampal eminence from its cephalic to its caudal extremity. From its beginning in the frontal region, the alveal system maintains a close relation with the under surface of the corpus callosum. Beneath the splenium and caudal portion of the callosal truncus, the crossing fibers of the alveus increase in number to form the massive hippocampal commissure.

Attention has already been called to the extent of the hippocampal eminence, which makes its appearance well forward in the body of the lateral ventricle. The ventricular protrusion in this region is not associated with the inversion of the hippocampal fissure, but rather with the extensive ingrowth into the ventricle of fibers entering the alveal system. The hippocampal eminence assumes it greatest prominence in relation to the trigonum ventriculi and the temporal horn of the ventricle. It is, of course, in these regions that the hippocampal structures acquire their largest dimensions and here also, the hippocampal fissure is the deepest and so occasions the greatest degree of archicortical inrolling into the ventricle.

The hippocampal eminence as a whole is generally pyriform in shape with its apex in the body of the lateral ventricle above the foramen of Monro and its base in the trigonum and temporal horn. In contour, the 
eminence is generally smooth and without surface irregularities. Nothing in its configuration justifies the recognition of subdivisions such as Hippocampus major and minor, Pes hippocampi, Calcar avis or any other surface feature meriting special designation.

With the exception of two regions, the subrostral and postcallosal flexures, the hippocampal fissure exhibits great depth. It may be traced from one end of the hippocampus to the other, without interruption. The fimbrio-dentate fissure is well marked in the subventricular, trigonal and temporal flexures.

All of these hippocampal features in the tapir establish a common bond of resemblance among the lower forms of mammals. It is only in the case of higher mammalian brains, such as those of the higher primates, that any notable departure from the common ground plan exists. This departure is apparently caused by the rapid and extreme growth of the frontal lobe in the monkeys, higher apes and man.

\section{Equus}

The reconstruction of the hippocampal formation in the horse, as in the tapir, shows the essential characteristics of this region. The model, unfortunately, is incomplete in its cephalic portion because the serial sections in the frontal area were too defective to permit reconstruction (fig. $23 B$ ). In other respects, the features of the hippocampus closely resemble those of the tapir. The subrostral, supracallosal, postcallosal and subcallosal flexures occupy their usual positions in the midline hippocampal division. Two characteristics of the hippocampal fissure are noteworthy, namely, a depth in the subrostral portion of the fissure greater than in the tapir and a striking fissural prominence in the postcallosal flexure.

Comment has already been made of the tendency for the hippocampal fissure in many mammals to become extremely shallow in the postcallosal region. This tendency may be an individual variant in all mammals or it may be a highly characteristic feature in certain species. In any event, the postcallosal portion of the hippocampal fissure in the specimen here presented is extremely deep and distinct.

The fissure extends forward beneath the corpus callosum where it continues to hold a position close to the midline. It then turns outward at right angles and is finally deflected backward to become a distinguishing landmark in the subventricular flexure.

The hippocampal fissure of the horse has its greatest depth in the long trigonal flexure (fig. $23 B$ ). It also represents a deep archicortical inrolling in the temporal flexure. As in other mammals examined, the boundaries of this fissure are the same. The dentate area forms one lip and the subicular area, the other. 
The six typical areas of the archicortex have their greatest structural distinctness in the divergent division of the hippocampus, but the general histological features of the several different archicortical areas may be observed in all parts of the midline hippocampal division as well. That portion of the hippocampus, therefore, in intimate relation with the corpus callosum, bears all the identifying features of the archicortex. These features are, however, less outstanding than in the hippocampal regions which have no callosal contact. The differences between the midline and divergent divisions of the hippocampus are thus not in the kind but in the degree of development. The reasons for regarding the midline hippocampal division, although developmentally retarded, homodynamous with the more fully developed divergent division, have previously been discussed with reference to the growth of the pig brain (page 48 ).

Another feature in the close structural correspondence between the two hippocampal divisions, is the presence of a long and slender cord of nervefibers which lies mesial to the dentate area in the subrostral, supracallosal, postcallosal and subcallosal flexures. Topographically and in other respects this cord of fibers appears to be related to the fimbrial system just as the more massive bundle in the subventricular, trigonal and temporal flexures is of this system.

In cross-section, the fimbrial fibers in the midline division of the hippocampus have the same disposition as in the tapir. They appear as two long, parallel cords of nerve fibers forming the median and lateral striae. At the cephalic extremity of the subcallosal flexure these two fiber-strands become continuous with the septal portion of the fimbria.

The alveal system makes its first appearance as part of the hippocampal eminence which projects into the lateral ventricle immediately in front of the foramen of Monro. It forms a long sheet of nerve fibers which covers the eminence from its small cephalic protrusion into the ventricle to its greatly expanded portion in the trigonum and temporal horn.

In all of its chief features, including fiber systems, cortical areas, divisions and relations, the hippocampus of these two perisodactyles, corresponds so closely that the same descriptive review may be applied to both of them.

\section{Giraffa camelopardalis}

The reconstruction of the hippocampal region in the giraffe (fig. 25) is incomplete in the frontal region. Its features in the more caudal areas of this huge artiodactyle ungulate bear a striking resemblance to those of the horse and the tapir.

The continuity of the hippocampal fissure may be traced in its relations to the supracallosal, postcallosal, and subcallosal flexures. As in 
the horse, the postcallosal portion of the fissure is deep and, with increasing depth, is continued into the subventricular, trigonal and temporal flexures.

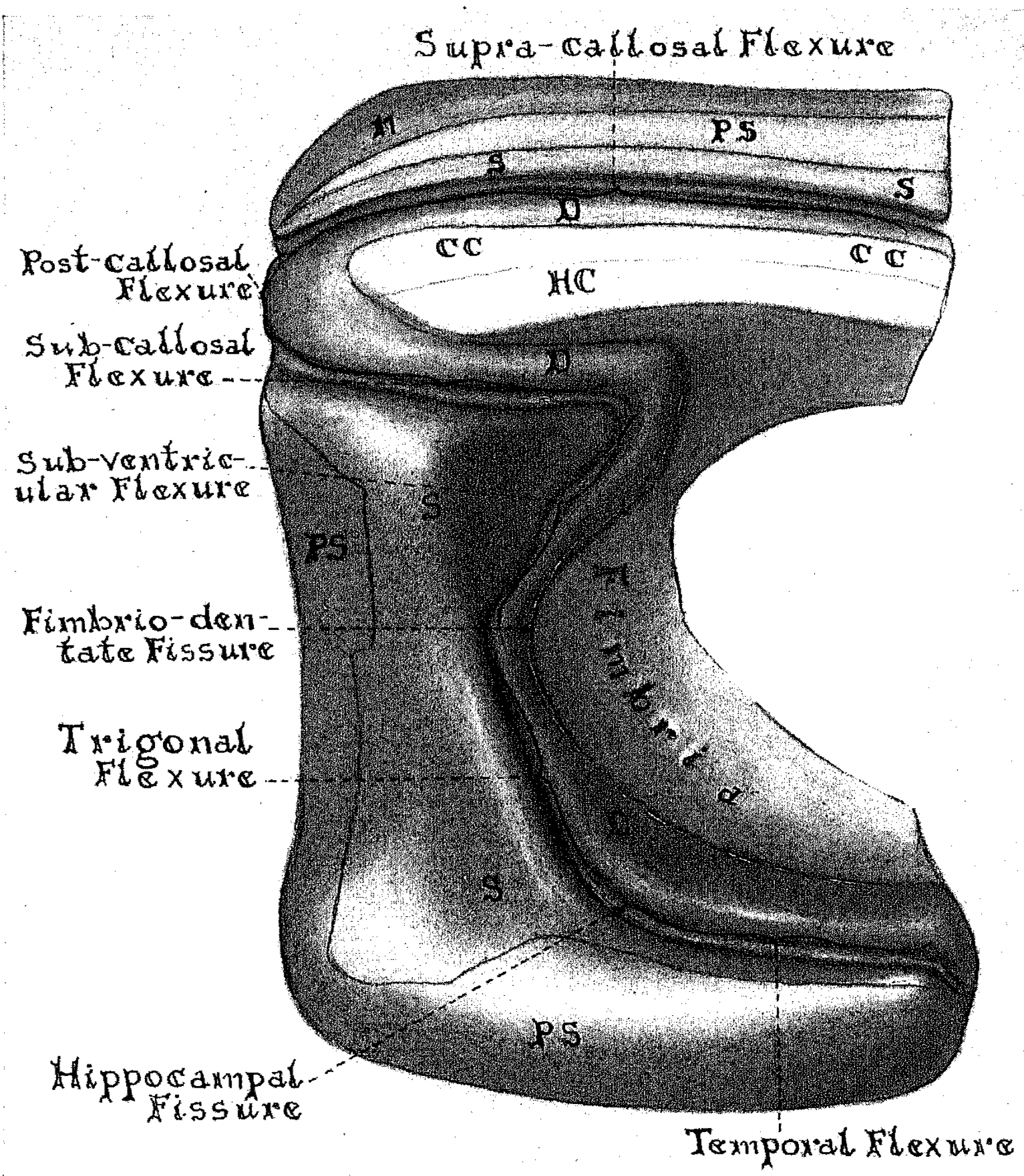

Fia, 25. Reconstruction of Hippocampal Regron in Adult Giraffe, Lemt HEMISPHERE

Specimen No. 256. Magnification $\times 5 . \quad c c$, corpus callosum; $D$, dentate area; $F$, fasciolar area; $H C$, hippocampal commissure; $n$, neocortex; $P S$, presubicular area; $S$, subicular area. 
The two hippocampal loops, which apparently characterize all mammals except the higher primates, are prominent in the giraffe. The loop forming part of the midline division of the hippocampus is well developed, and its junction with the divergent hippocampal division produces the contour typical of this region of the brain.

In one particular the hippocampus in the giraffe is different from that in either the horse or tapir; the fibers entering the alveal system project much farther outward into the body of the lateral ventricle where they form a long intraventricular shelf. This feature may be a characteristic of the artiodactyle ungulates as it also occurs in the pig's brain. Any conclusion of this kind, however, must depend on the microscopic examination of a much greater number of artiodactyles than the present material affords.

\section{Felis domesticus}

The corpus callosum in the reconstruction of the cat brain (fig. 26), shows a well developed rostrum, genu, truncus and splenium. The septum is thick. Its mesial surface is composed of nerve fibers continuous with the fimbrial system which pass downward behind the anterior commissure.

The relations of the two fiber systems in the cat are similar to those in most lower mammals. Alveal fibers cover the hippocampal eminence and enter the hippocampal commissure which forms a massive bundle beneath the corpus callosum. Fimbrial fibers form a long arching bundle which extends upward from the temporal region and then forward and downward into the septum. This bundle in its caudal portion corresponds to the so-called "posterior pillar of the fornix," the arched portion, to the "body of the fornix," and the septal portion, to "the anterior pillar of the fornix."

These two great fiber-systems, the alveus and fimbria, bear their characteristic relations. The alveus in its greater part is intraventricular while the fimbria is extraventricular. Both systems maintain contact with each other in the region of the chorioidal fissure, along which line there appears to be some interchange of fibers between them.

Eight typical hippocampal flexures, including the subrostral, precallosal, supracallosal, postcallosal, subcallosal, subventricular, trigonal and temporal, are well defined in the cat's brain. The unitary histological nature of the cortical tissue throughout all of these flexures is attested by the appearance of the six characteristic areas of the archicortex which occur and are disposed in their usual relations in all parts of the hippocampus.

The hippocampal fissure, with the dentate area forming one boundary and the subicular area the other, extends without interruption from the subrostral region to the splenium. Directly behind the splenium, the 
fissure becomes somewhat shallow, but again assumes great depth in the subcallosal area. The hippocampal fissure has greatest prominence in the subventricular, trigonal and temporal areas. In these latter areas the fimbrio-dentate fissure is most conspicuous but it may be identified, even if somewhat indistinct in all parts of the hippocampus.

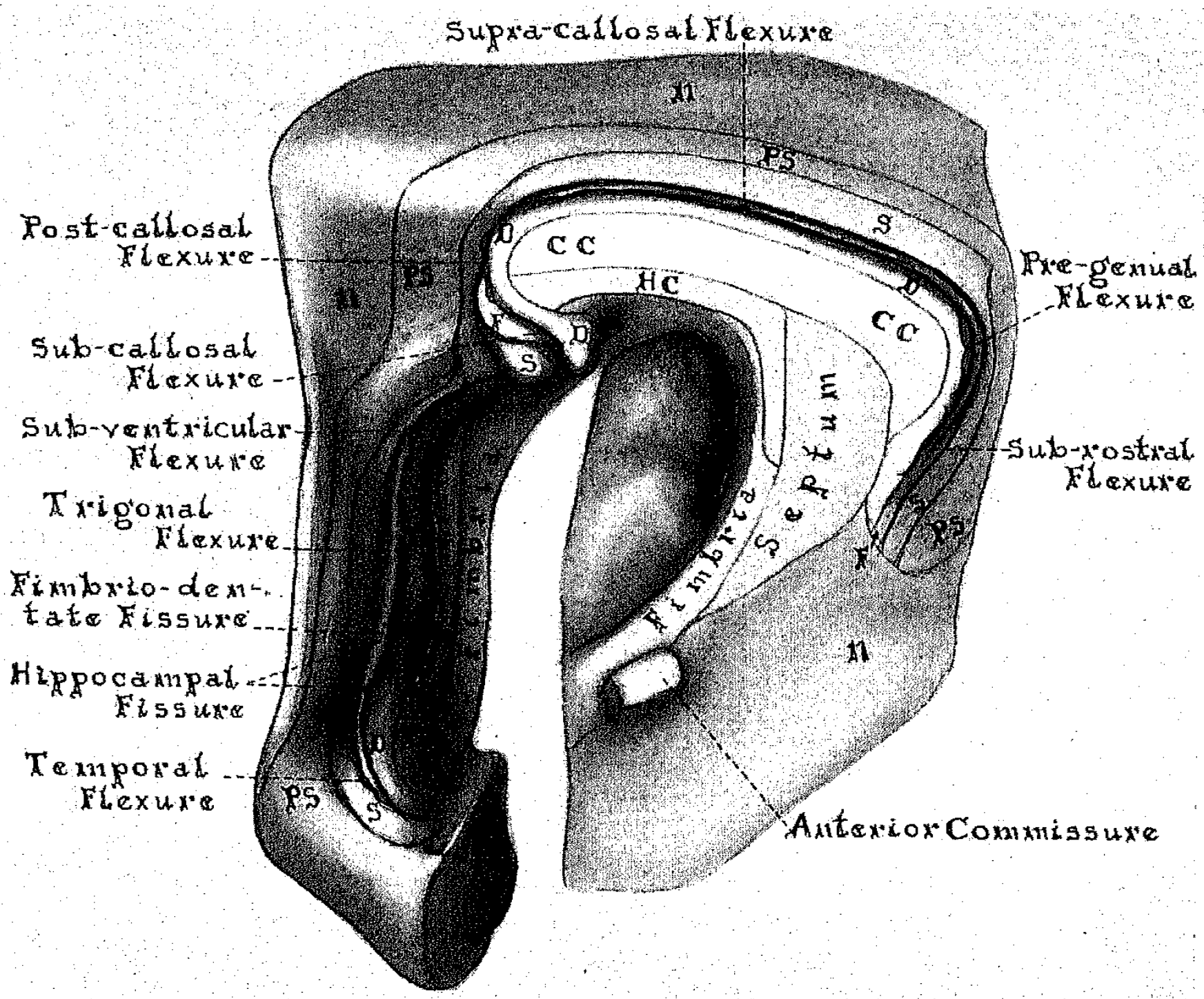

Fig. 26. Reconstruction Showing Hippocampal Region of Lefr Hemisphere of AdULT Cat

Specimen No. 230. Magnification $\times 10 . c c$, corpus callosum; $D$, dentate area; $F$, fasciolar area; $H C$, hippocampal commissure; $n$, neocortex; $P, S$, presubicular
area; $S$, subicular area.

The midline hippocampal division shows the usual histological retardations incident to its contacts with the corpus callosum. On the other hand, the divergent division gives evidence of extensive expansion apparently in consequence of its access to the lateral ventricle.

The hippocampal eminence makes its first appearance in the body of the lateral ventricle immediately in front of the foramen of Monro. It 
has its usual pyriform contour, with apex pointing forward and base lying in the trigonum and temporal horn. It is covered by the thin subependymal sheet of fibers which forms the alveus.

Fibers, which now may be referred to as constituting the midline fimbria, have their characteristic arrangement in forming the mesial stria along the dorsum of the corpus callosum.

In a small portion of the trigonal region, the fasciolar area occupies an extraventricular position due to the marked imrolling of the archicortex. An extraventricular alveus is present in this same area.

\section{Scallops}

The reconstruction of the hippocampus in the common mole reveals the identifying characteristics in this region of the insectivore brain. The corpus callosum is relatively short and thin. More than half of it lies in the anterior quadrant, i.e., in front of the foramen of Monro. The rostrum and genu are poorly developed and consequently the subrostral and precallosal flexures of the hippocampus are not as conspicuous as in most other mammals (fig. 27). The anterior commissure is unusually large.

Another peculiarity in the mole is the great thickness of the septum and the massive fiber system which constitutes the entire septal surface of the mesial wall. This system is continuous with the fibers forming the alveus and the fimbria.

The hippocampal fissure forms, as in other mammals, the identifying landmark for the hippocampal formation. It begins as a relatively shallow groove immediately in front of the callosal genu and extends backward without interruption through the supracallosal flexure. Continuing its course as a still deeper groove, the fissure passes downward behind the splenium and then, for a short distance, forward in relation with the subcallosal flexure. It is, however, in the subventricular, trigonal and temporal flexures that the hippocampal fissure acquires its greatest depth.

The eight flexures of the hippocampus are all well defined, although the subrostral and subcallosal flexures are less extensive than in other mammals.

Six typical areas of the archicortex are distinguishable and bear their usual relations to the corpus callosum and the hippocampal fissure.

In the midline division of the hippocampus, the archicortical areas have their characteristic retardation in histological specialization. The divergent division, on the other hand, shows, in a pronounced manner, the expansive effects due to proximity to the lateral ventricle as well as the avoidance of growth limiting influences occasioned by contact with the corpus callosum. The dentate area in this division of the hippocampus 
is especially conspicuous because of its unusual size. It is, in fact, of far greater surface extent than in any other mammal examined.

The hippocampal eminence has its typical pyriform contour with its apex well forward in the body of the lateral ventricle and its greatly expanded base protruding into the trigonum ventriculi and temporal horn.

Longitudinal bundles of nerve-fibers form the median and lateral striae which run their usual course in relation to the corpus callosum and fimbria.

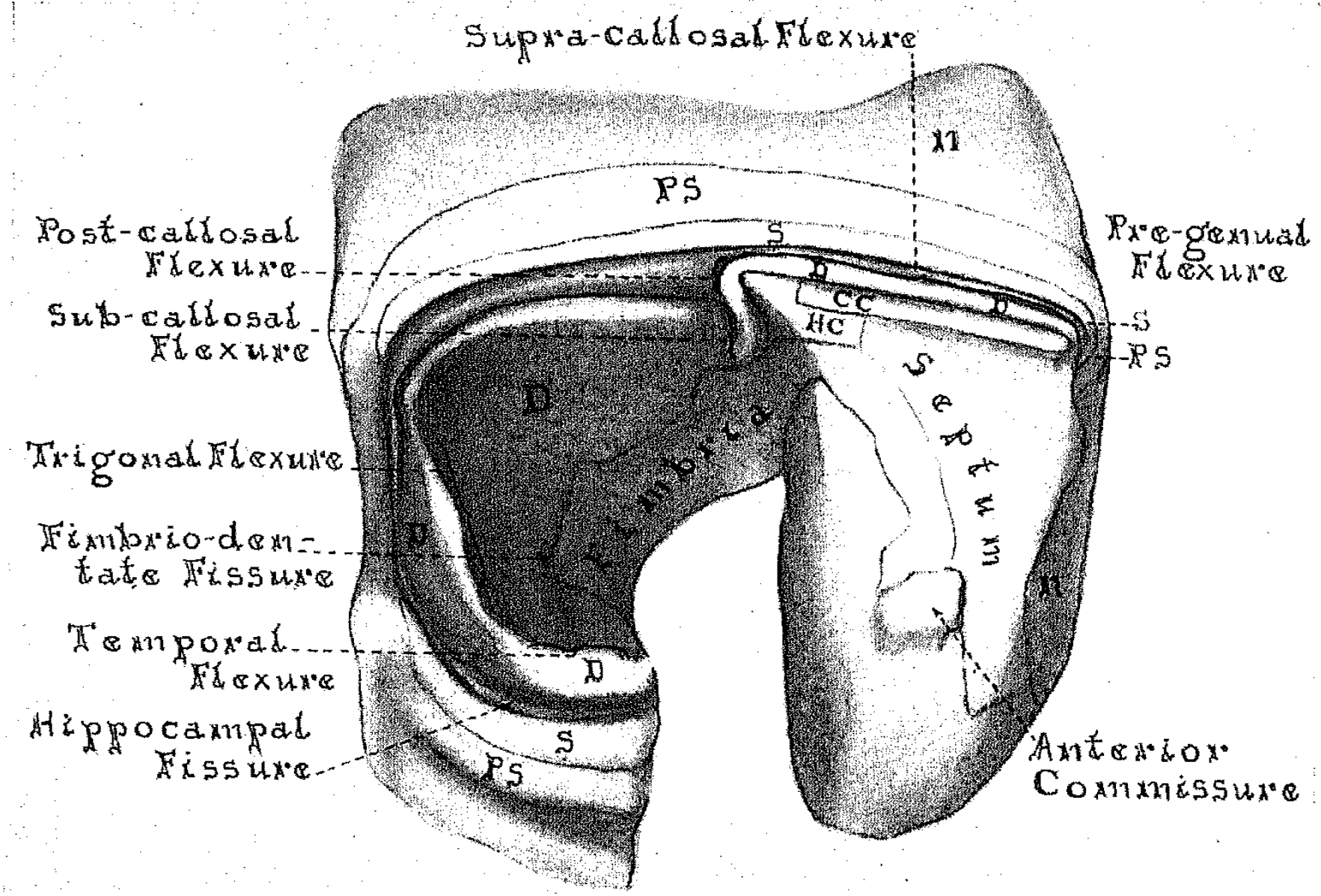

Fig. 27. Reconstruction of Left Hippocampal Region in Mole

Specimen No. 263. Magnification $\times 20$. cc, corpus callosum; $D$, dentate area; $F$, fasciolar area; $H C$, hippocampal commissure; $n$, neocortex; $P S$, presubicular area;
$S$, subicular area.

\section{Lemur mongoz}

Occupying a position on the lowest level of the primates, the lemur, as might be expected, retains many characteristics of lower mammals. Such retention is especially apparent in the hippocampus, nor is it surprising that this ancient feature of the brain should show so little of progressive advancement in animals definitely on the borderline between the simpler and most highly developed mammals.

The descriptions already given for the horse, tapir, cat and mole, might, with a few modifications, be equally well applied to the lemur. The one 
great characteristic of the higher primate brain has not yet made the weight of its influence felt upon these transitional members of the order. The consequences of great expansions in the frontal and parietal areas are still to be fortheoming.

The corpus callosum (fig. 28) in the lemur has all of the typical features of this structure in eutherian mammals, including its several recognized parts, the rostrum, genu, truncus and splenium as well as its relations to

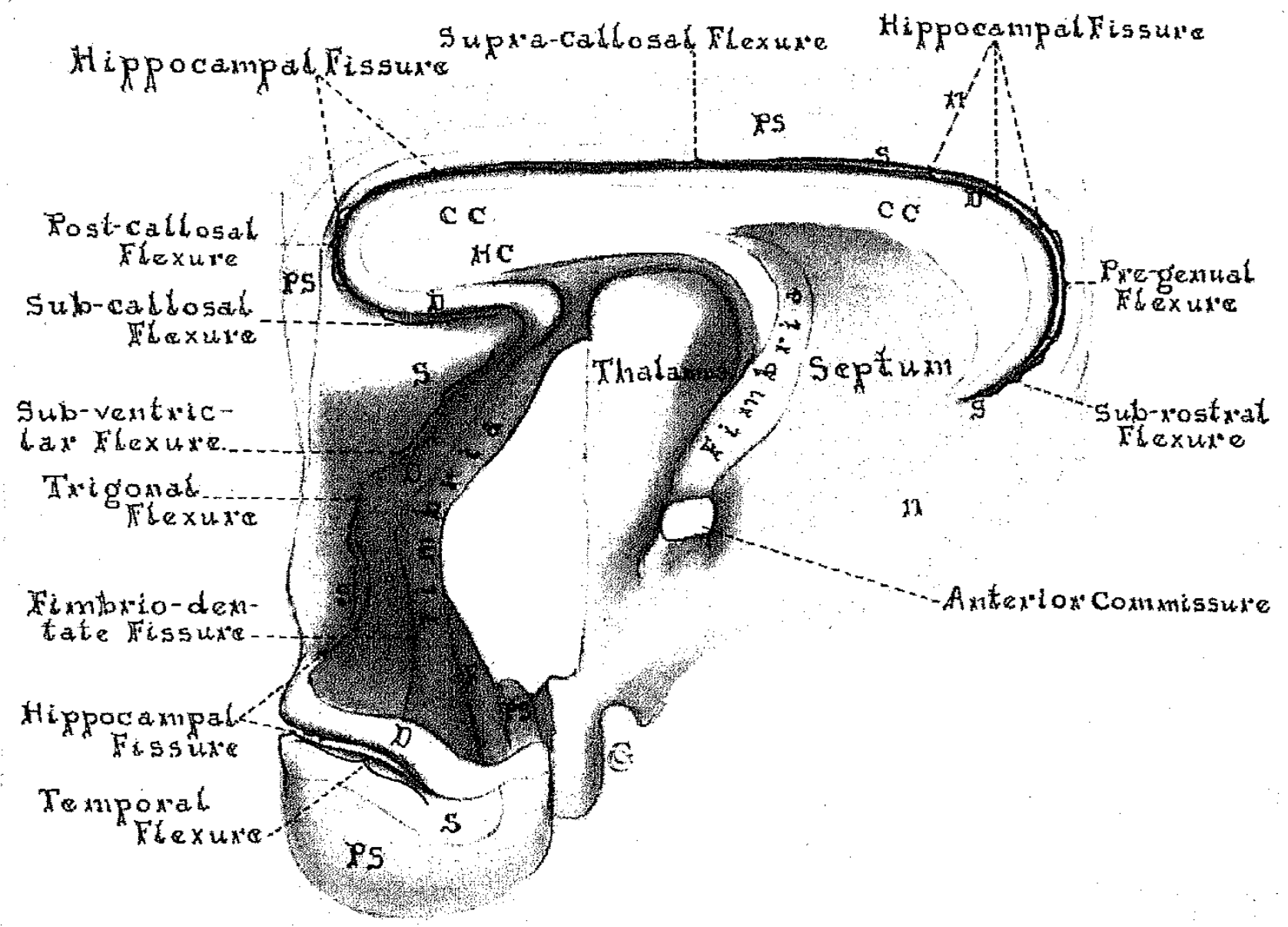

Fig. 28. Reconstruction of Hippocampal Formation of Left Hemispifere in LEMUR MONGOZ

Specimen No. 147. Magnification $\times 10$. $c c$, corpus callosum; $D$, dentate area; $F$, fasciolar area; $H C$, hippocampal commissure; $n$, neocortex; $P S$, presubicular area; $S$, subicular area.

the hippocampal commissure and to the midline division of the hippocampus.

The midline and divergent hippocampal divisions are well defined and the two loops, one passing around the end of the splenium and the other extending outward beneath the body of the lateral ventricle, are conspicuous in the lemur. Again, as in other mammals described, the influences of the corpus callosum and thalamus upon the hippocampal structures are clear. 
The lemur is in close correspondence with other mammals in the number of its hippocampal flexures and its archicortical areas. Eight flexures affect the hippocampus; these include the subrostral, precallosal, supracallosal, postcallosal, subcallosal, subventricular, trigonal and temporal flexures. Throughout its entire extent the hippocampal formation presents a unitary histological appearance. The archicortex in all regions of the hippocampus shows the six typical areas, namely, the presubicular, subicular, crescentic, fasciolar, intercalary and dentate.

These areas have their usual arrangement with reference to the hippocampal fissure, the subicular area forming one boundary of the fissure, the dentate area, the other. The fissure, as in other instances, is most shallow in the subrostral flexure. Its depth in the postcallosal flexure is considerable, while it becomes deepest in the trigonal and temporal flexures. A well developed fimbrio-dentate fissure occurs in the divergent division of the hippocampus.

The fimbria and alveus have their usual positions and relations. Nothing in the reconstruction of the lemur seems to justify the designation of "pillars of the fornix". The fiber system forming the inner margin of the hippocampus constitutes the fimbria, the anterior portion of which is incorporated in the septum, and is thus without clear surface delineation.

What may be spoken of as the midline fimbria occupies the characteristic position and relations on the dorsum of the corpus callosum.

The hippocampal eminence is characteristically situated in the body, trigonum and temporal horn of the lateral ventricle. It is typically pyriform in contour with a long apex which begins immediately in front of the foramen of Monro. No surface markings make any special subdivisions distinguishable.

In a small portion of the hippocampus near the base of the trigonal flexure, the fasciolar area is partially extraventricular; a correspondingly small portion of the alveus is also extraventricular.

\section{Primate changes in the brain which effect the hippocampus}

In primates more advanced than the lemurs, certain progressive modifications of the brain have had a profound influence upon the hippocampus. These changes do not wait upon the appearance of the highest anthropoid apes, like the gorilla and chimpanzee. They begin to develop in the humbler South American monkeys. In essence, they consist of specific enlargements in the cerebral hemispheres, of expansions in particular areas of the neocortex.

Chief in importance are the increments, both in size and definiteness, which occur in the frontal lobe. But these expansions are also attended by marked additional growth in the parietal and temporal lobes. The increasing expansions in these three highly important lobes occasion a 
corresponding increase in the number of nerve cells and nerve fibers in the brain so that the needs of interhemispheral communication become greater. Effects of such growth are ultimately reflected upon the corpus callosum which consequently gains considerably in length. In the main, this elongation appears in the callosal trunk, with the result that the distance between the genu and splenium is disproportionately longer than in lower mammals.

From such evidence as is available, it seems clear that the corpus callosum begins to develop in the frontal region, in front of the foramen of Monro and the anterior commissure. It then grows backward above the dorsal surface of the optic thalamus.

The early accession of callosal fibers from the frontal, parietal and temporal lobes would thus tend to carry the splenium caudad in such a way as to bear with it the hippocampal commissure and at the same time cause an elongation of the longitudinal fibers of the fimbria.

It would be interesting to know what adaptive modifications in behavior lie behind these changes in the primate brain. Whatever they may have been and however much open to controversy, it is certain that they began to take effect early in the history of the most primitive monkeys. Tree life and the development of quadrumanous specializations are not to be minimized as decisive influences which bore great weight in the expansions of the frontal and parietal lobes. From these sources of increased cerebral power radiated many secondary structural modifications, among which were the changes in the hippocampus.

In the primitive South American monkeys, which in addition to four hand-like extremities, possess a prehensile tail often spoken of as a "fifth hand", the frontal and parietal lobes are already well developed. Mycetes, the howling monkey, is a good example of this group. Effects of frontal, parietal and temporal expansions are visible in the corpus callosum and especially in the hippocampus. Not only is the callosal elongation conspicuous, but the hippocampal changes are even more noteworthy. In all monkeys and apes these changes are of a similar character and restricted to similar regions of the hippocampus. They affect the postcallosal, trigonal and temporal regions almost exclusively and consist of (1) a nearly complete suppression of the subcallosal flexure; (2) a marked abbreviation of the subventricular fexure; (3) an oblique instead of a perpendicular course of the postcallosal flexure in descending from the midline downward and outward to join the subventricular flexure; (4) the appearance of irregular dentations in the dentate area of the trigonal flexure; and (5) the beginning of the dentate retroversion which forms the uncus. All of these hippocampal modifications have their inception in mycetes. The prominent loop, which in lower mammals is formed by the subcallosal and subventricular flexures, does not appear. The obliquity 
at which the postcallosal flexure passes across the splenium is still further increased in the baboon with the result that the hippocampal fissure passes diagonally across the splenium. This disposition of the fissure in the baboon is also true in macacus rhesus and in the gibbon, orang outang, chimpanzee and gorilla.

The reconstruction of the hippocampal region in the gorilla represents the conditions in all of the anthropoid apes, proanthropoids and lower monkeys.

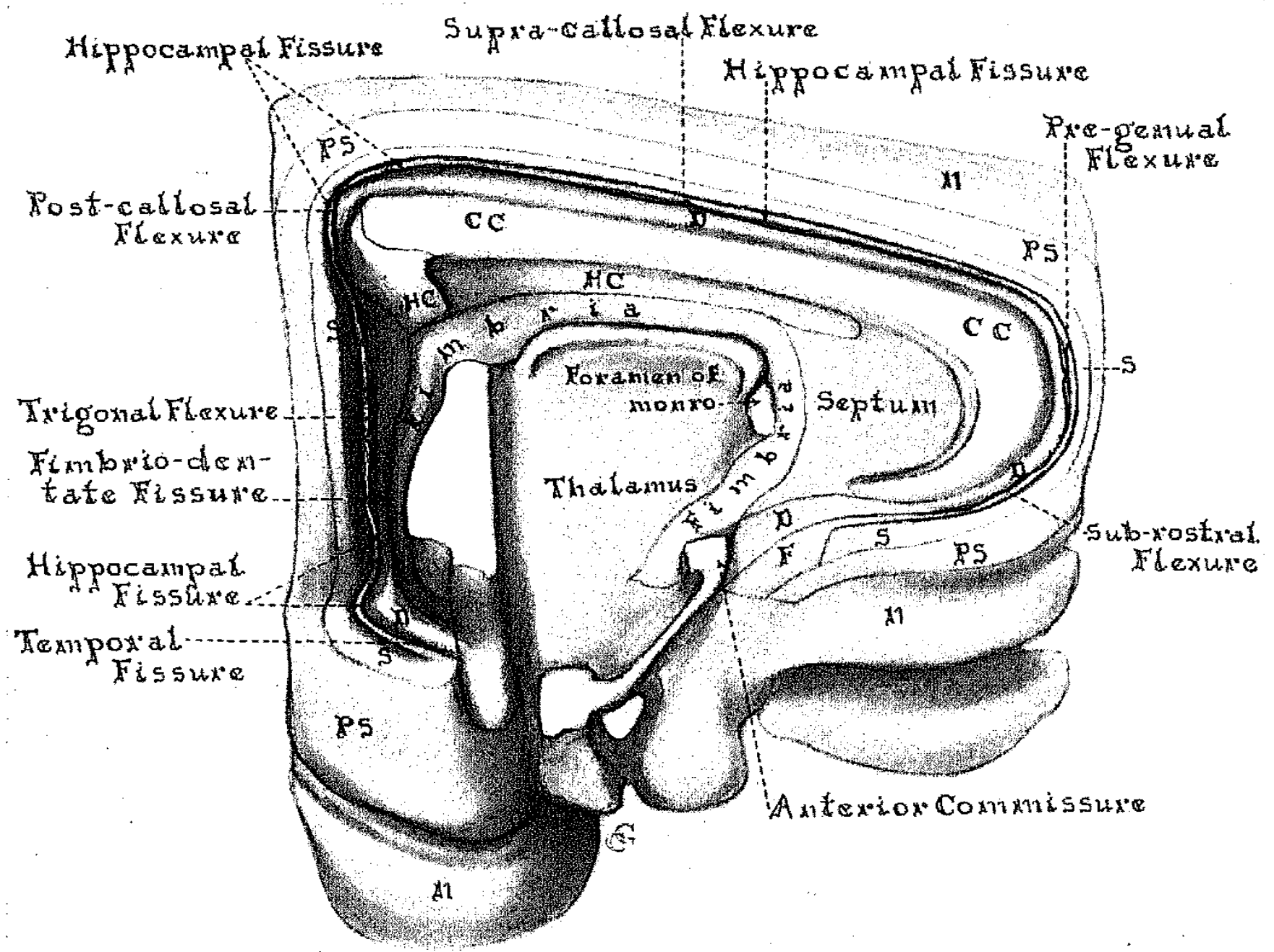

Fig. 29. Reconstruction of Lefry Hippocampat Region in Gorilida

Specimen No. 246. Magnification $\times 5$. cc, corpus callosum; $D$, dentate area; $F$, fasciolar area; $H C$, hippocampal commissure; $n$, neocortex; $P S$, presubicular area; $S$, subicular area.

\section{Gorilla}

The corpus callosum in the gorilla (fig. 29) shows in marked degree the elongation of the truncus. There is a similar lengthening of the rostrum as well as of the septum, which now is referred to as the septum pellucidum because of its reduction in thickness due to the development of a well defined cavum pellucidum. The cavum is, in general, a primate characteristic in the septal region. It is poorly defined in most of 
the lower primates but a prominent feature in all of the great anthropoids and man. In subprimate mammals, the septum is heary and thick; it has but little translucency and is either devoid of a cavum altogether or develops irregular and unconnected lacunae between the otherwise adherent leaves of the right and left septa. A lateral and a mesial set of nerve fibers constitute the main bulk of the septum but between them is interspersed an irregular mass of gray matter containing small groups of nerve cells of different sizes.

The elongated corpus callosum tends to emphasize the prominence of several parts of the neocortical commissure and gives the rostrum, the genu, the truncus and the splenium even sharper definition than in lower mammals. The splenium, as usual, consists of two groups of crossing fibers, namely, the caudalmost callosal fibers and the fibers of the hippocampal commissure. The latter structure appears less extensive than in the general run of animals.

While the midline hippocampal division has most of the characteristics common to other species, including the several flexures of the hippocampus, the six typical archicortical areas, the hippocampal fissure and the midline fimbria, it shows a significant departure in the region of the splenium. This departure involves the postcallosal flexure which descends obliquely downward and outward to join a greatly shortened subventricular flexure. Furthermore the subcallosal flexure, by the caudal extension of the splenium, has been suppressed so that the loop of archipallium which offers identifying features of the hippocampus in lower mammals, does not appear in gorilla. Shortening of the subventricular flexure in conjunction with this suppression of the subcallosal flexure produces a more direct and less circuitous passage of the midline into the divergent hippocampus. These structural modifications are undoubtedly secondary changes due to the elongation of the corpus callosum.

In estimating the morphological significance of these secondary modifcations, the expansions especially in the frontal and parietal lobes should not alone be taken into account. The obvious cephalo-caudal elongation of the optic thalamus is also an important factor. In all probability it exerts its influence in consequence of growth in the neothalamus and corresponding increases in the volume of thalamo-cortical pathways, particularly to the frontal and parietal areas.

Because of changes induced by the increased size of the frontal and parietal lobes, the number of flexures in the hippocampus is actually reduced in the gorilla. Instead of eight such flexures characteristic of most other species, there are in the gorilla but seven. The subcallosal flexure does not appear and the subventricular flexure is much reduced in size. On the other hand, the six archicortical areas maintain all of their typical distinctness and relations to the hippocampal and fimbrio-dentate fissures. 
The alveus and fimbria retain their usual relations but, due to the callosal elongation, the fimbria has more of the appearance associated with generally accepted descriptions of the fornix. The body of this structure which is long and slender, arches above the dorsal surface of the thalamus; its anterior pillar, more discretely defined than in most lower species, gives the impression of a distinct support to the vault of the fornix. The descending or posterior pillar of the formix, constituting the portion of the fimbrial system in relation with the trigonum ventriculi and temporal horn, likewise appears as a pillar supporting the arch of the fornix. However satisfying this conception of the fimbrial system may be in gorilla and other higher primates, it is only with difficulty and many objections that it may be applied in lower mammals. Furthermore its very succintness obscures, to a considerable extent, at least, the important fact that the fornix, so called with some justification in man and the higher primates, represents the fimbria and all that this great system of fibers signifies in the organization of the hippocampus.

The midline fimbria, occupying its relations to the so-called indusium griseum, starts in the subrostral flexure and encircles the genu and truncus of the corpus callosum. After passing across the splenium it enters the subcallosal portion of the fimbria.

The hippocampal eminence is chiefly prominent in the trigonum ventriculi and in the temporal horn. It has a slender extension which projects forward into the body of the lateral ventricle and terminates a short distance in front of the foramen of Monro. This projection consists exclusively of fibers of the alveal and fimbrial systems. The alveal fibers are in immediate contact and continuous with the under surface of the corpus callosum. Dependent from the alveal fibers and forming a shelf which projects into the lateral ventricle, the fibers of the fimbria extend forward and finally sweep downward in front of the foramen of Monro to form the anterior pillar of the fornix.

The hippocampal eminence is generally regular in outline and presents no special surface markings which merit special designation.

In a small region near the base of the trigonal flexure, the fasciolar area and alveus are extraventricular.

\section{Types of hippocampal formation in the mammalian brain}

The three major types of hippocampal formation are well represented in the opossum, the elephant and man. Certain modifications or variants, which may, perhaps, deserve the title of subtypes, are easily comprehended within this classification. The identifying distinctions are in all instances the same.

In the first place, there are the features which distinguish between the midline and divergent divisions of the hippocampus, whether in the non- 
callosal or callosal mammal. In either case, the great expansion of the optic thalamus produces a definite divergence of the hippocampal formation in the region of the brain caudal to the foramen of Monro and the anterior commissure.

Secondly, there is the fact that, wherever the hippocampus has free access to the lateral ventricle, it undergoes characteristic expansions, whereas it exhibits retardation whenever it is prohibited from such access.

Thirdly, the fundamental hippocampal flexures, however modified or complicated by the interpolation of the corpus callosum, may be identified in all mammals.

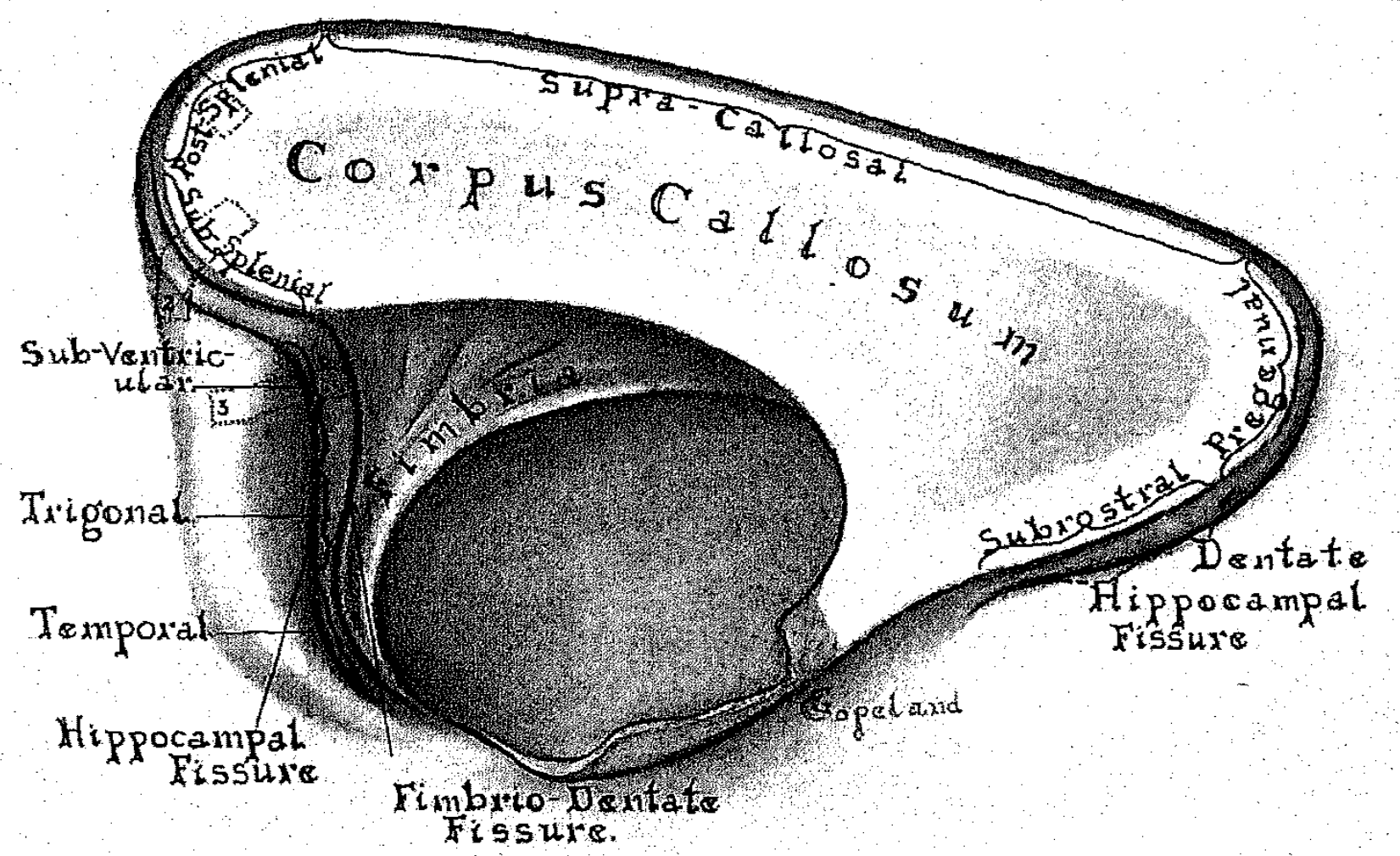

Fit. 30. Gross Dissietion of Fippocampal Region in Adult African Elempant Specimen No. 110. Magnification $\times 2$

The hippocampal formation in the opossum has already been considered in detail and its appearance in the adult shown in figure 13.

In the gross dissection of the African elephant, (Elephas Africanus) the hippocampus shows all the effects incident to the introduction of the corpus callosum which as usual consists of a rostrum, genu, truncus and splenium (fig. 30).

As in most eutherian mammals, the splenium comprises a large ventral group of fibers forming the hippocampal commissure and a dorsal segment representing commissural fibers from the neocortex.

The hippocampus displays its eight characteristic flexures, including the subrostral, the precallosal (pregenual), the supracallosal, the postcallosal 
(postsplenial), the subcallosal (subsplenial), the subventricular, the trigonal and the temporal flexure.

The midline hippocampal division, due to the presence of the corpus callosum, has all the features typical of this structure in the eutheria. The main portion of the hippocampus is excluded from the body of the lateral ventricle by the interposition of callosal fibers.

The divergent division of the hippocampus beneath the splenium swings sharply away from the midline and in consequence forms well defined subcallosal and subventricular flexures. In this manner, the two hippocampal loops, so characertistic of most callosal brains, are clearly outlined. The vertical loop, comprised of the supracallosal and the postcallosal flexure passes around the splenium. It joins the horizontal loop, consisting of the subventricular flexure, by means of the subcallosal flexure (fig. 30).

The hippocampal fissure may be traced, without interruption, through each of the several flexures from one end of the hippocampus to the other. In the left hemisphere of this particular specimen, the fissure becomes very shallow. It is discernable however, and may be followed into the subcallosal flexure, where it again assumes greater depth. Sections of the postcallosal region, in which the hippocampal fissure has become so shallow as to create the impression that it actually terminates at this point, reveal how unwarranted such a supposition is. As a matter of fact these sections show that all of the boundaries of the fissure are present. The fissural floor, consisting of the crescentic area, has been raised almost to the general level of the mesial surface of the hemisphere and has thus considerably increased the distance between the usual boundaries of the hippocampal fissure, namely the dentate area on one side and the subicular area on the other.

This tendency on the part of the fissure in the postcallosal region, to be deep or shallow may offer further morphological criteria for the recognition of certain subtypes in the classification of the mammalian hippocampus. In this light it would be possible to identify sub-types in which the hippocampal fissure is either extremely shallow or extremely deep, in the postcallosal region of both hemispheres or shallow in one hemisphere and deep in the other. While such suggestions may prove to have little or no importance, it nevertheless seems desirable to draw attention to them because of the possible physiological or morphological significance which they may represent.

Gross dissections of several other mammals, including the black bear (Ursus Americanus), wolf (Canis occidentalis), calf (Bos taurus), Aoudad (Ammotragus lervia), sheep (Ovis aries) and llama (Llama vicugna), together with others previously considered, justify the conclusion that there is a type of hippocampus characteristic of eutherian mammals exclusive of the higher primates. The chief features of this type of hippo- 
campal formation reveal themselves both in the midline and divergent divisions. They are especially notable in the formation of the postcallosal, subcallosal and subventricular flexures as well as in those two remarkable loops of the hippocampus, one of which sweeps around and under the splenium while the other extends out laterally beneath the body of the lateral ventricle to become continuous with the trigonal flexure.

Still a third type of hippocampal formation, already indicated in the descriptions of the anthropoid apes, may be found in the gross dissection of the human brain. The dissection in this instance is of the left hemisphere of a two months old child (fig. 31). The most noteworthy features

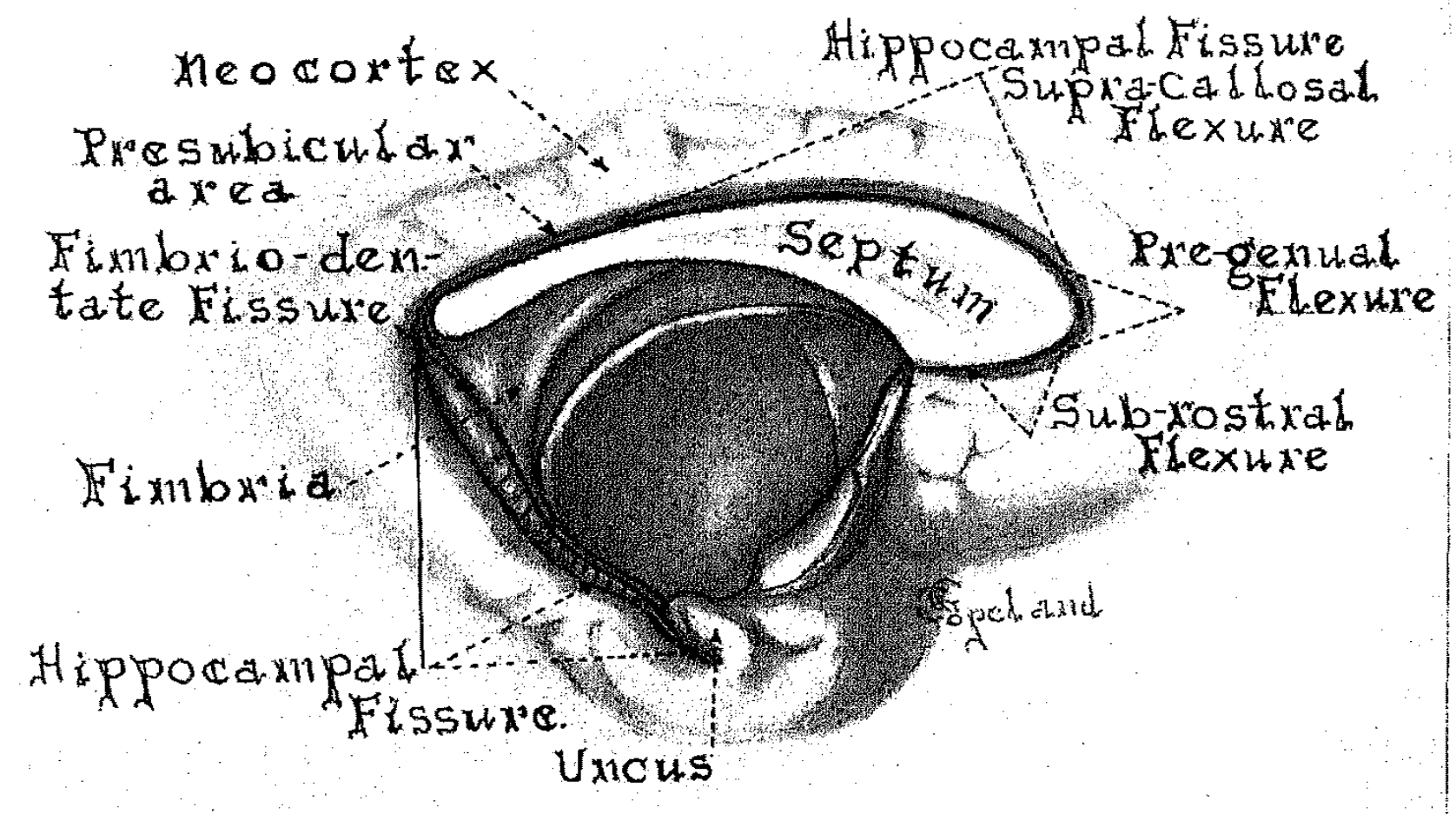

Fig. 31. Gross Dissection of Hippocampal Rigion in Human Infant, Two Months Old. Specimen No. 2007

here are the marked cephalo-caudal lengthening of the corpus callosum and the suppression of both the subcallosal and subventricular flexures. The postcallosal flexure passes obliquely downward and outward to join the trigonal flexure. The lengthening of the corpus callosum in response to the increased size in the frontal, parietal and temporal lobes has already been discussed. The probable effects of this callosal lengthening on the position of the hippocampal commissure, on the postcallosal, the subcallosal and subventricular flexures has also been considered.

These changes in man appear to result in the loss of two of the usual flexures of the hippocampus, but the development of the uncus at the extreme cephalic end of the temporal flexure introduces an element which makes its appearance in the most advanced anthropoids and man. Added 
to this uncal flexure, is the marked dentation in the dentate area. Such a toothed-like configuration is not unfamiliar in lower mammals. Usually it affects the subicular rather than the dentate area. Only in the anthropoid apes does it become at all conspicuous, while its prominence in man is undoubtedly the reason for calling this region the "fascia dentata".

The three major types of hippocampal formation, as it occurs in mammals, are therefore; 1 , the marsupial type characteristic of mammalian brains before the corpus callosum has made its appearance, this type of hippocampus, in all probability, is also characteristic of the monotreme brain; 2, the general eutherian type which appears in all mammals possessing a corpus callosum up to and including the lemurs; and 3 , the primate type common to all monkeys, apes and higher anthropoids. It attains the culmination of its developmental refinement in man.

It is probable that this classification does not make provision for all of the possible types of hippocampus which this part of the brain may later be shown to embrace in mammals. Certain sub-types have already been suggested in this discussion, but it seems reasonably clear, from what is already known, that regardless of the number of hippocampal varieties and subvarieties, one constant, common feature will be found to run through them all, namely the unitary structural nature in the organization of the archipallium. Comprehended within this unitary nature of the archipallium are such preeminent features as the succession in parallel with each other of six archicortical areas including the presubicular, the subicular, the crescentic, the fasciolar, the intercalary and the dentate area; the histological constancy and specificity of these areas; the continuity of the hippocampal fissure in relation to these areas from one end of the hippocampus to the other; the constancy in the relations of the two great hippocampal fiber systems, the alveus and fimbria; and the effects of proximity to the lateral ventricle upon the development of the hippocampal formation.

The consideration of further details in the human hippocampus together with a critical review of the literature on the hippocampal formation and the corpus callosum will appear later in Part II of this paper.

\section{REFERENCES}

Arantius, Julius Caesar: De humano foetu. ... Ejusdem anatomicorum observationum liber, etc. Venetiis, 1587 , pp. 44-45.

DeGatengeot, Reń JaQues Croissant: Splanchnologie ou l'anatomie des viscères. 2e. edit., Osmont, Paris, 1742, T. 2: 250-251.

Duvernoi, Jo. Georg: De sinibus cerebri. Comm. acad, sci. imp. petropolitanae. Petropoli, 1735, Tom. 4: 130-135, ad annum 1729 .

Gưther: Kurzer Entwurf der anatomischen Nervenlehre. Düsseldorf, 1789, p. 19; Schreger. Synonymik der anatomischen Nomenclatur. Fürth, 1803.

His, Wruhecm: Die anatomische Nomenclatur. Arch. f. Anat. u. Entw., Supplement-Band, 1895, p. 86 and p. 170. 
HIs, Wrrhelm: Die anatomische Nomenclatur; Nomina anatomica. IX. Versammlung in Basel angenommenen Namen. Leipzig, Veit, 1895.

HYRTL, Joseph: Onomatologia anatomica. Wien, 1880, pp. 148-9 and 183.

Lewis, Frederic T.: The significance of the term hippocampus. J. Comp. Neurol, $1923,35: 213$.

Mayer, Johann C. A.: Anatomiseh-physiologische Abhandlung vom Gehirn, Rückenmark, und Ursprung der Nerven. Berlin u. Leipzig, 1779, p. 8.

Morand, Johannes F. C.: Observations anatomiques sur quelques parties du cerveau. Mém. de l'Acad. Roy, de Sci. de l'année 1744. Amsterdam, 1751, pp. $430-444$.

Roтн, M.: Andreas Vesalius. Berlin, 1892, p. 75.

VICQ D'AzUr, Frlix: Traité d'Anatomie et de Physiologie. Vocabulaire anatomique Hippocampe. Paris, 1786, 1: 55-123.

Willis, Thomas: Cerebri Anatome. Amstelodami, Londini, 1664.

Winslow, JACques BÉnIGNe: Exposition anatomique de la structure du corps humain. Paris, 1732, p. 619 . 